quatrième série-tome 41 fascicule 5 septembre-octobre 2008

$$
\begin{aligned}
& \text { ANNALES } \\
& \text { SCIENTIFIQUES } \\
& \text { de } \\
& \text { L'ECOLE } \\
& \text { NORMALE } \\
& \text { SUPÉRIEURE }
\end{aligned}
$$

Alberto S. CATTANEO \& Charles TOROSSIAN

$$
\begin{gathered}
\text { Quantification pour les paires symétriques } \\
\text { et diagrammes de Kontsevich }
\end{gathered}
$$


Ann. Scient. Éc. Norm. Sup.

$4^{\text {e }}$ série, t. 41,2008, p. 789 à 854

\title{
QUANTIFICATION POUR LES PAIRES SYMÉTRIQUES ET DIAGRAMMES DE KONTSEVICH
}

\author{
par Alberto S. CATtAneO et Charles TOROSSiAN
}

\begin{abstract}
RÉsumÉ. - In this article we use the expansion for biquantization described in [7] for the case of symmetric spaces. We introduce a function of two variables $E(X, Y)$ for any symmetric pairs. This function has an expansion in terms of Kontsevich's diagrams. We recover most of the known results though in a more systematic way by using some elementary properties of this $E$ function. We prove that Cattaneo and Felder's star product coincides with Rouvière's for any symmetric pairs. We generalize some of Lichnerowicz's results for the commutativity of the algebra of invariant differential operators and solve a long standing problem posed by M. Duflo for the expression of invariant differential operators on any symmetric spaces in exponential coordinates. We describe the Harish-Chandra homomorphism in the case of symmetric spaces by using all these constructions. We develop a new method to construct characters for algebras of invariant differential operators. We apply these methods in the case of $\sigma$-stable polarizations.
\end{abstract}

Abstract. - Dans cet article nous appliquons les méthodes de bi-quantification décrites dans [7] au cas des espaces symétriques. Nous introduisons une fonction $E(X, Y)$, définie pour toutes paires symétriques, en termes de graphes de Kontsevich. Les propriétés de cette fonction permettent de démontrer de manière unifiée des résultats importants dans le cas des paires symétriques résolubles ou quadratiques. Nous montrons que le star-produit décrit dans [7] coïncide, pour toute paire symétrique, avec celui de Rouvière. On généralise un résultat de Lichnerowicz sur la commutativité d'algèbres d'opérateurs différentiels invariants et on résout un problème de M. Duflo sur l'écriture, en coordonnées exponentielles, des opérateurs différentiels invariants sur tout espace symétrique. On décrit l'homomorphisme d'Harish-Chandra en termes de graphes de Kontsevich. On développe une théorie nouvelle pour construire des caractères des algèbres d'opérateurs différentiels invariants. On applique ces méthodes dans le cas des polarisations $\sigma$-stables.

\section{Introduction}

Let $(\mathfrak{g}, \sigma)$ be a symmetric pair: viz., $\mathfrak{g}$ is a finite-dimensional Lie algebra over $\mathbb{R}$, while $\sigma$ is an involution and a Lie algebra automorphism of $\mathfrak{g}$. We denote by $\mathfrak{g}=\mathfrak{k} \oplus \mathfrak{p}$ the decomposition relative to $\sigma$, with $\mathfrak{k}$ and $\mathfrak{p}$ the +1 - and -1 -eigenspaces, respectively (Cartan's decomposition). 
The Poincaré-Birkhoff-Witt (PBW) theorem ensures the following decomposition of the universal enveloping algebra $U(\mathfrak{g})$ :

$$
U(\mathfrak{g})=U(\mathfrak{g}) \cdot \mathfrak{k} \oplus \beta(S(\mathfrak{p}))
$$

with $\beta$ the symmetrization map from $S(\mathfrak{g})$ into $U(\mathfrak{g})$. ${ }^{(1)}$ One can then identify, as vector spaces, the symmetric algebra $S(\mathfrak{p})$ of $\mathfrak{p}$ with $U(\mathfrak{g}) / U(\mathfrak{g}) \cdot \mathfrak{k}$ via $\beta$. Though $U(\mathfrak{g}) / U(\mathfrak{g}) \cdot \mathfrak{k}$ is not an algebra in general, its $\mathfrak{k}$-invariant subspace $(U(\mathfrak{g}) / U(\mathfrak{g}) \cdot \mathfrak{k})^{\mathfrak{k}}$ is an algebra.

This is of fundamental importance as it is the algebra of invariant differential operators on the symmetric space $G / K$ associated to the symmetric pair $(\mathfrak{g}, \sigma)$, and as such it occurs in harmonic analysis on symmetric spaces in a crucial way.

This algebra is commutative [21, 12], and the PBW theorem ensures that

$$
(U(\mathfrak{g}) / U(\mathfrak{g}) \cdot \mathfrak{k})^{\mathfrak{k}} \text { and } S(\mathfrak{p})^{\mathfrak{k}}
$$

are isomorphic as vector spaces. Observe that $S(\mathfrak{p})^{\mathfrak{k}}$ is just the associated graded of $(U(\mathfrak{g}) / U(\mathfrak{g}) \cdot \mathfrak{k})^{\mathfrak{k}}$.

It is conjectured [32] that these two algebras are isomorphic as algebras, what the second author has called the polynomial conjecture. This is a generalization for symmetric pairs of Duflo's isomorphism [11] between the center $U(\mathfrak{g})^{\mathfrak{g}}$ of the universal enveloping algebra of a Lie algebra $\mathfrak{g}$ and the invariant subalgebra $S(\mathfrak{g})^{\mathfrak{g}}$ of its symmetric algebra.

Recall that for every homogeneous space $G / H$ the algebra of $G$-invariant differential operators may be identified, thanks to a result of Koornwinder [20], with the algebra $(U(\mathfrak{g}) / U(\mathfrak{g}) \cdot \mathfrak{h})^{\mathfrak{h}}$ (where $\mathfrak{h}$ denotes the Lie algebra of $H$ ). The latter is not commutative in general. Its associated graded is then a Poisson subalgebra of $(S(\mathfrak{g}) / S(\mathfrak{g}) \cdot \mathfrak{h})^{\mathfrak{h}}$ with a natural Poisson structure.

If $\mathfrak{h}$ admits a complement $\mathfrak{q}$ which is invariant under the adjoint action of $\mathfrak{h}$, then an easy consequence of PBW is that $(U(\mathfrak{g}) / U(\mathfrak{g}) \cdot \mathfrak{h})^{\mathfrak{h}}$ and $(S(\mathfrak{g}) / S(\mathfrak{g}) \cdot \mathfrak{h})^{\mathfrak{h}}$ are still isomorphic as vector spaces. It is not known whether this holds in general, for there is no natural map from $(S(\mathfrak{g}) / S(\mathfrak{g}) \cdot \mathfrak{h})^{\mathfrak{h}}$ to $U(\mathfrak{g})$ (or a quotient thereof).

It has been conjectured anyway by M. Duflo [13] that the center of $(U(\mathfrak{g}) / U(\mathfrak{g}) \cdot \mathfrak{h})^{\mathfrak{h}}$ and the Poisson center of $(S(\mathfrak{g}) / S(\mathfrak{g}) \cdot \mathfrak{h})^{\mathfrak{h}}$ are always isomorphic as algebras.

Little is known in such generality. In case $\mathfrak{g}$ is a nilpotent Lie algebra, appreciable advances have been achieved in the last few years by Corwin-Greenleaf [10], Fujiwara-LionMagneron-Mehdi [14], Baklouti-Fujiwara [5] and Baklouti-Ludwig [6]. In case where $G$ and $H$ are reductive groups, F. Knop [18] gives a satisfying and remarkable answer to the conjecture. In case where $H$ is compact and $G=H \triangleright<N$ is the semidirect product of $H$ with a Heisenberg group $N$, Rybnikov [30] makes use of Knop's result to prove Duflo's conjecture in that case.

In this paper we propose a novel approach to these questions based on Kontsevich's construction [19] and its extension to the case of coisotropic submanifolds by Cattaneo and

(1) We have $\beta\left(X_{1} \ldots X_{n}\right)=\frac{1}{n !} \sum_{\sigma \in S_{n}} X_{\sigma(1)} \ldots X_{\sigma(n)}$. 
Felder [7], [8]. We only treat the problem of symmetric pairs here, but we think that our methods have a wider scope, namely in the nilpotent homogeneous case ${ }^{(2)}$.

One may regard the present work as a link between the methods of deformation quantization and the orbit method in Lie theory.

\section{Plan of the paper}

In Section 1 we recall Kontsevich's construction for the deformation quantization of Poisson manifolds and its extension by Cattaneo and Felder to the case of coisotropic submanifolds. We discuss in details the compatibility in cohomology. This section should be useful for the Lie algebra experts who are not familiar with the deformation quantization constructions.

Next we study the dependency of this construction on the choice of a complement in the linear case. We show that the reduction spaces are isomorphic and describe the isomorphism (Proposition 2 and Theorem 1) which is an element of the gauge group obtained by solving a differential equation.

In Section 2 we describe the graphs appearing in the linear case and present three fundamental examples of reduction spaces occurring in Lie theory: symmetric pairs (Proposition 4), Iwasawa's decompositions (Proposition 5), and polarizations (Proposition 6). These examples are new and show that this novel quantization methods are well-suited for Lie theory.

Recall that for symmetric pairs F. Rouvière [26, 27, 29], following Kashiwara and Vergne [17], introduced a mysterious function $e(X, Y)$ defined for $X, Y \in \mathfrak{p}$. Up to conjugation this function computes the star product

$$
\underset{\text { Rou }}{\sharp} Q=\beta^{-1}(\beta(P) \cdot \beta(Q) \text { modulo } U(\mathfrak{g}) \cdot \mathfrak{k})
$$

for $P, Q$ in $S(\mathfrak{p})^{\mathfrak{k}}$.

In Section 3 we define a function $E(X, Y)$ for $X, Y \in \mathfrak{p}$ in terms of graphs. This function will behave as Rouvière's function $e(X, Y)$. The comparison of these two functions is a key point of this paper. The function $E(X, Y)$ expresses the Cattaneo-Felder star product $\stackrel{\star}{\star F}$ in the case of symmetric pairs. By inspection of the graphs appearing in its construction, we obtain a symmetry property (Lemma 11) together with some additional properties in the solvable case (Proposition 8), in the case of Alekseev-Meinrenken symmetric pairs (Proposition 10) as well as in the case of very symmetric quadratic pairs (Proposition 11). In all these cases we show that the function $E$ is identically equal to 1 . These elementary but remarkable properties yield new and uniform proofs of results obtained by Rouvière in the solvable case (Theorem 2, Section 3.4 and Proposition 9) and generalize a theorem by Alekseev and Meinrenken (Theorem 3) in the (anti-invariant) quadratic case.

In Section 4 we show that the Cattaneo-Felder and the Rouvière star products coincide (Theorem 4). This is a new result. From this we deduce (Theorem 5) the commutativity for all $z \in \mathbb{R}$ of the algebras $\left(U(\mathfrak{g}) / U(\mathfrak{g}) \cdot \mathfrak{k}^{z \operatorname{tr}_{k}}\right)^{\mathfrak{k}}$ of invariant differential operators on $z$-densities, thus generalizing a result by Duflo [12] and Lichnerowicz [21].

(2) One of our students is working on this case. 
Another problem posed by M. Duflo in [13] is solved in Section 4: the expression in exponential coordinates of invariant differential operators (Theorem 6). Our solution is given in terms of Kontsevich's graphs.

We define at the end of Section 4 a deformation along the axis of the Campbell-Hausdorff formula for symmetric pairs in the spirit of the Kashiwara-Vergne conjecture. We proved (Theorem 7 and Proposition 13) that this deformation in the case of quadratic Lie algebras, considered as very symmetric quadratic pairs, implies the Kashiwara-Vergne conjecture. We conjecture that in the case of Lie algebras considered as symmetric pairs our $E$ function is equal identically to 1 . This conjecture would solve the Kashiwara-Vergne conjecture.

In Section 5 we consider the Harish-Chandra homomorphism for symmetric pairs. Actually there are two natural choices for a complement of $\mathfrak{k}^{\perp}$, one by Cartan's decomposition and the other by Iwasawa's. We show that these two choices together lead to the HarishChandra homomorphism for general symmetric pairs. In this language the Harish-Chandra homomorphism consists of the restriction to the little symmetric pair in Iwasawa's decomposition. The former decomposition and the intertwinement then yield a formula for the Harish-Chandra homomorphism in terms of graphs. It follows from this expression that the Harish-Chandra homomorphism is invariant under the action of the generalized Weyl group. We hope that this formula will allow a resolution of the polynomial conjecture for symmetric pairs.

Finally, in Section 6 we apply the principles of bi-quantization [7] to the case of triplets $f+\mathfrak{b}^{\perp}, \mathfrak{g}^{*}, \mathfrak{k}^{\perp}$, where $\mathfrak{b}$ is a polarization for $f \in \mathfrak{k}^{\perp}$. These constructions produce characters for the algebras of invariant differential operators (Proposition 19). It is a novel method that we hope will be promising in other situations as well ${ }^{(3)}$.

In the case of polarizations in normal position we show, by a homotopy on the coefficients (which makes use of an 8 color form), that the characters are independent of the choice of polarization (Proposition 21). Thus we recover some classical results of the orbit method for Lie algebras.

It follows that for symmetric pairs admitting $\sigma$-stable polarizations Rouvière's isomorphism computes the characters of the orbit method (Theorem 8).

One can regard these new methods as a replacement for the orbit method.

\section{Introduction}

Soit $(\mathfrak{g}, \sigma)$ une paire symétrique, c'est-à-dire $\mathfrak{g}$ est une algèbre de Lie (quelconque) de dimension finie sur $\mathbb{R}$ et $\sigma$ est une involution qui est un automorphisme d'algèbres de Lie. On note alors $\mathfrak{g}=\mathfrak{k} \oplus \mathfrak{p}$ la décomposition relative à $\sigma$, où $\mathfrak{k}$ désigne l'espace propre associé à la valeur propre +1 et $\mathfrak{p}$ l'espace propre associé à la valeur propre -1 . Cette décomposition est aussi appelée décomposition de Cartan.

Le théorème de Poincaré-Birkhoff-Witt (PBW) assure la décomposition de l'algèbre enveloppante $U(\mathfrak{g})$ :

$$
U(\mathfrak{g})=U(\mathfrak{g}) \cdot \mathfrak{k} \oplus \beta(S(\mathfrak{p})),
$$

(3) These methods may be applied in certain cases of homogeneous spaces.

$4^{\text {e }}$ SÉRIE - TOME $41-2008-\mathrm{N}^{\mathrm{o}} 5$ 
où $\beta$ désigne la symétrisation de $S(\mathfrak{g})$ dans $U(\mathfrak{g}) .{ }^{(4)}$ On peut alors identifier $S(\mathfrak{p})$ l'algèbre symétrique de $\mathfrak{p}$ et $U(\mathfrak{g}) / U(\mathfrak{g}) \cdot \mathfrak{k}$ via la symétrisation $\beta$. En général $U(\mathfrak{g}) / U(\mathfrak{g}) \cdot \mathfrak{k}$ n'est pas une algèbre, mais les $\mathfrak{k}$-invariants $(U(\mathfrak{g}) / U(\mathfrak{g}) \cdot \mathfrak{k})^{\mathfrak{k}}$ forment une algèbre.

Cette algèbre est un objet central car c'est l'algèbre des opérateurs différentiels invariants sur l'espace symétrique $G / K$ associé à la paire symétrique $(\mathfrak{g}, \sigma)$. Elle intervient de manière cruciale dans l'analyse harmonique sur les espaces symétriques.

Cette algèbre est commutative $[21,12]$ et le théorème de Poincaré-Birkhoff-Witt nous assure que

$$
(U(\mathfrak{g}) / U(\mathfrak{g}) \cdot \mathfrak{k})^{\mathfrak{k}} \text { et } S(\mathfrak{p})^{\mathfrak{k}}
$$

sont isomorphes comme espaces vectoriels. Remarquons que $S(\mathfrak{p})^{\mathfrak{k}}$ est tout simplement le gradué associé de $(U(\mathfrak{g}) / U(\mathfrak{g}) \cdot \mathfrak{k})^{\mathfrak{k}}$.

On conjecture [32] que ces deux algèbres sont isomorphes comme algèbres, ce que le second auteur a nommé la conjecture polynomiale. Cette conjecture généralise pour les paires symétriques l'isomorphisme de Duflo pour les algèbres de Lie [11] entre le centre de l'algèbre enveloppante $U(\mathfrak{g})^{\mathfrak{g}}$ et les invariants dans l'algèbre symétrique $S(\mathfrak{g})^{\mathfrak{g}}$.

Précisons dans cette introduction que pour un espace homogène $G / H$ quelconque (on note $\mathfrak{h}$ l'algèbre de Lie de $H$ ) l'algèbre des opérateurs différentiels invariants sous l'action de $G$ s'identifie grâce à un résultat de Koornwinder [20] à l'algèbre $(U(\mathfrak{g}) / U(\mathfrak{g}) \cdot \mathfrak{h})^{\mathfrak{h}}$. Cette algèbre n'est pas commutative en général. Son gradué associé est alors une sous-algèbre de Poisson de $(S(\mathfrak{g}) / S(\mathfrak{g}) \cdot \mathfrak{h})^{\mathfrak{h}}$ (cette dernière possède une structure de Poisson naturelle).

S'il existe un supplémentaire de $\mathfrak{h}$ qui soit invariant sous l'action adjointe de $\mathfrak{h}$, notons-le $\mathfrak{q}$, alors une conséquence facile de PBW est que $(U(\mathfrak{g}) / U(\mathfrak{g}) \cdot \mathfrak{h})^{\mathfrak{h}}$ et $(S(\mathfrak{g}) / S(\mathfrak{g}) \cdot \mathfrak{h})^{\mathfrak{h}}$ sont encore isomorphes comme espaces vectoriels. En général, on ne sait pas si cette propriété reste vraie, pour la simple raison qu'il n'existe pas d'application naturelle de $(S(\mathfrak{g}) / S(\mathfrak{g}) \cdot \mathfrak{h})^{\mathfrak{h}}$ dans $U(\mathfrak{g})$ (ou un quotient).

On conjecture toutefois que le centre de $(U(\mathfrak{g}) / U(\mathfrak{g}) \cdot \mathfrak{h})^{\mathfrak{h}}$ et le centre de Poisson de $(S(\mathfrak{g}) / S(\mathfrak{g}) \cdot \mathfrak{h})^{\mathfrak{h}}$ sont toujours isomorphes comme algèbres : c'est une conjecture de M. Duflo [13].

Peu de choses sont connues dans cette généralité. Dans le cas où $\mathfrak{g}$ est une algèbre nilpotente, des progrès sensibles ont été faits ces dernières années par Corwin-Greenleaf [10], Fujiwara-Lion-Magneron-Mehdi [14], Baklouti-Fujiwara [5] et Baklouti-Ludwig [6]. Dans le cas où $G$ et $H$ sont des groupes réductifs, F. Knop [18] donne une réponse satisfaisante et remarquable à cette conjecture. Dans le cas ou $H$ est compact et $G=H \ltimes N$ est un produit semi-direct de $H$ par un groupe d'Heisenberg $N$, Rybnikov [30] utilise le résultat de Knop pour conclure positivement à la conjecture de Duflo.

Cet article propose une approche nouvelle sur ces questions, basée sur la construction de Kontsevich [19] et ses extensions aux cas des sous-variétés co-isotropes par Cattaneo-Felder [7], [8]. Nous abordons ici la problématique des paires symétriques mais nous pensons que

(4) On a $\beta\left(X_{1} \ldots X_{n}\right)=\frac{1}{n !} \sum_{\sigma \in S_{n}} X_{\sigma(1)} \ldots X_{\sigma(n)}$. 
nos méthodes ont un champ d'applications plus vaste, notamment dans le cas nilpotent homogène ${ }^{(5)}$.

On peut voir ce mémoire comme un pont entre les méthodes de quantification par déformation et la méthode des orbites en théorie de Lie.

Résultats détaillés de l'article. - La section 1 rappelle les constructions de quantification de Kontsevich et l'extension au cas des variétés co-isotropes par Cattaneo-Felder. On détaillera l'argumentation sur la compatibilité en cohomologie. Cette section est utile pour les experts en algèbre de Lie qui ne sont pas familiers avec les constructions de quantification par déformation.

On étudie ensuite dans le cas linéaire la dépendance de ces constructions par rapport au choix du supplémentaire. Nous montrons que les espaces de réduction sont isomorphes et nous décrivons l'isomorphisme (Proposition 2 et Théorème 1) : c'est un élément du groupe de jauge qui s'obtient via la résolution d'une équation différentielle.

Dans la section 2, on précisera les graphes qui interviennent dans le cas linéaire et on donnera trois exemples fondamentaux d'espaces de réduction que l'on peut rencontrer en théorie de Lie : le cas des paires symétriques (Proposition 4), le cas des décompositions d'Iwasawa (Proposition 5) et le cas des polarisations (Proposition 6). Ces exemples sont nouveaux et démontrent que ces nouvelles méthodes de quantification sont adaptées à la théorie de Lie.

Rappelons que dans une série d'articles, F. Rouvière [26, 27, 29] a introduit pour les paires symétriques, suivant les méthodes de Kashiwara-Vergne [17], une fonction mystérieuse $e(X, Y)$ définie pour $X, Y \in \mathfrak{p}$. Cette fonction calcule, à une conjugaison près, le star-produit :

$$
\underset{\text { Rou }}{\sharp} Q=\beta^{-1}(\beta(P) \cdot \beta(Q) \text { modulo } U(\mathfrak{g}) \cdot \mathfrak{k})
$$

pour $P, Q$ dans $S(\mathfrak{p})^{\mathfrak{k}}$.

Dans la section 3, on définira une fonction $E(X, Y)$ pour $X, Y \in \mathfrak{p}$ en termes de graphes, qui aura des propriétés analogues à la fonction $e(X, Y)$ de Rouvière. La comparaison de ces deux fonctions est un point important de cet article. Cette fonction $E(X, Y)$ exprime le star-produit $\underset{C F}{\star}$ de Cattaneo-Felder dans le cas des paires symétriques. On déduira, à partir des graphes intervenant dans la construction de cette fonction, une propriété de symétrie (Lemme 11) et des propriétés supplémentaires dans le cas résoluble (Proposition 8), dans le cas des paires symétriques d'Alekseev-Meinrenken (Proposition 10) ou le cas très symétrique quadratique (Proposition 11). Dans tous ces cas on montre que la fonction $E$ vaut identiquement 1 . Ces propriétés remarquables et élémentaires donnent des démonstrations nouvelles et unifiées de résultats démontrés par Rouvière dans le cas résoluble (Théorème 2 et Proposition 9) et généralisent un théorème d'Alekseev-Meinrenken (Théorème 3) dans le cas quadratique (anti-invariant).

Dans la section 4, on montrera que le star-produit $\underset{C F}{\star}$ de Cattaneo-Felder et celui de Rouvière coïncident (Théorème 4). Ce résultat est nouveau. On en déduira (Théorème 5) la commutativité pour tout $z \in \mathbb{R}$ des algèbres d'opérateurs différentiels invariants sur les $z$-densités $\left(U(\mathfrak{g}) / U(\mathfrak{g}) \cdot \mathfrak{k}^{z \operatorname{tr}_{k}}\right)^{\mathfrak{k}}$ généralisant ainsi un résultat de Duflo [12] et Lichnerowicz [21].

(5) Un des nos étudiants travaille sur ce cas.

$4^{\mathrm{e}}$ SÉRIE - TOME $41-2008-\mathrm{N}^{\mathrm{o}} 5$ 
Un autre problème, proposé par M. Duflo dans [13], est résolu dans la section 4 : l'écriture, en coordonnées exponentielles, des opérateurs différentiels invariants (Théorème 6). Notre réponse s'exprime en termes de graphes de Kontsevich.

En fin de section 4, on définit une déformation le long des axes de la formule de CampbellHausdorff pour les paires symétriques, dans l'esprit de la conjecture de Kashiwara-Vergne. On montre (Théorème 7 et Proposition 13) que dans le cas des algèbres de Lie quadratiques, considérées comme des paires très symétriques quadratiques, notre déformation implique la conjecture de Kashiwara-Vergne. Plus généralement on conjecture que notre fonction $E$ vaut 1 dans le cas des algèbres de Lie, considérées comme des paires symétriques. Cette conjecture implique alors la conjecture de Kashiwara-Vergne.

Dans la section 5, on s'intéresse à l'homomorphisme d'Harish-Chandra pour les paires symétriques. En effet il existe deux choix de supplémentaires de $\mathfrak{k}^{\perp}$, essentiellement celui donné par la décomposition de Cartan et l'autre donné par la décomposition d'Iwasawa. On montre que ces deux choix conduisent à l'homomorphisme d'Harish-Chandra pour les paires symétriques générales. Dans ce langage, l'homomorphisme d'Harish-Chandra consiste en la restriction à la petite paire symétrique dans la décomposition d'Iwasawa. La première décomposition et l'entrelacement donneront alors une formule pour l'homomorphisme d'HarishChandra en termes de graphes. On en déduit au vu de l'expression que l'homomorphisme d'Harish-Chandra généralisé est invariant par l'action du groupe de Weyl généralisé. On espère que cette formule permettra de résoudre la conjecture polynomiale pour les paires symétriques.

Enfin dans la section 6 de cet article, on appliquera le principe de bi-quantification [7] au cas des triplets $f+\mathfrak{b}^{\perp}, \mathfrak{g}^{*}, \mathfrak{k}^{\perp}$ où $\mathfrak{b}$ est une polarisation pour $f \in \mathfrak{k}^{\perp}$. Ces constructions fournissent des caractères pour les algèbres d'opérateurs différentiels invariants (Proposition 19). C'est une nouvelle méthode, que l'on espère prometteuse dans d'autres situations ${ }^{(6)}$.

Dans le cas où les polarisations sont en position d'intersections normales, on montre, par une méthode d'homotopie sur les coefficients (faisant intervenir une forme à 8 couleurs), que les caractères sont indépendants du choix des polarisations (Proposition 21). On retrouve ainsi des résultats classiques de la méthode des orbites dans le cas des algèbres de Lie.

On en déduit, pour les paires symétriques qui admettent des polarisations $\sigma$-stables, que l'isomorphisme de Rouvière calcule les caractères de la méthode des orbites (Théorème 8).

On peut voir ces nouvelles méthodes comme un substitut à la méthode des orbites.

\section{Rappels sur la construction de Cattaneo-Felder}

Soit $\mathfrak{g}$ une algèbre de Lie de dimension finie sur $\mathbb{R}$. L'espace dual $\mathfrak{g}^{*}$ est alors muni d'une structure de Poisson linéaire. On note $\pi$ le bi-vecteur de Poisson associé.

Supposons donnée une sous-algèbre $\mathfrak{h}$ de $\mathfrak{g}$. Son orthogonal $\mathfrak{h}^{\perp}$ est alors une sous-variété co-isotrope de $\mathfrak{g}^{*}$. Dans [7], Cattaneo et Felder décrivent une construction pour une quantification de $(S(\mathfrak{g}) / S(\mathfrak{g}) \cdot \mathfrak{h})^{\mathfrak{h}}$ ou d'une sous-algèbre de cette dernière.

(6) Ces méthodes s'appliquent dans certains cas d'espaces homogènes. 


\subsection{Quantification}

Les constructions ne sont pas intrinsèques et dépendent du choix d'un supplémentaire de $\mathfrak{h}$ dans $\mathfrak{g}$. Notons $\mathfrak{q}$ un tel supplémentaire. On peut alors identifier $\mathfrak{h}^{*}$ avec $\mathfrak{q}^{\perp}$.

Deux constructions sont données par Cattaneo et Felder, l'une en termes de série de type Feynman avec diagrammes colorés et l'autre en terme de transformée de Fourier partielle. Ces constructions sont locales mais on peut les globaliser. On va rappeler ces dernières dans le cadre qui nous intéresse à savoir le cas des sous-algèbres, mais il suffira de remplacer $\mathfrak{h}^{\perp}$ par une sous-variété $C$ pour obtenir la construction plus générale.

Rappel des constructions. - La variété qui intervient dans cette construction est une supervariété :

$$
M:=\mathfrak{h}^{\perp} \oplus \Pi \mathfrak{h},
$$

où $\Pi$ désigne le foncteur de changement de parité. L'algèbre des fonctions est donc

$$
\mathcal{A}:=\mathcal{C}^{\infty}\left(\mathfrak{h}^{\perp}\right) \otimes \bigwedge\left(\mathfrak{g}^{*} / \mathfrak{h}^{\perp}\right) \simeq \mathcal{C}^{\infty}\left(\mathfrak{h}^{\perp}\right) \otimes \bigwedge \mathfrak{h}^{*}
$$

Si $C=\mathfrak{h}^{\perp}$, la fibre du fibré normal $N_{C}$ vaut alors $\mathfrak{g}^{*} / \mathfrak{h}^{\perp}$, celle du fibré cotangent (conormal) $T_{C}^{\perp}=N_{C}^{*}$ vaut $\mathfrak{h}$.

\subsection{Construction en terme de transformée de Fourier}

La dg-algèbre des poly-champs sur $M$ est l'algèbre symétrique décalée de l'algèbre des dérivations de $\mathcal{A}$

$$
\mathcal{T}(A):=S_{\mathcal{A}}(\operatorname{Der}(\mathcal{A})[-1])[1] .
$$

Par transformée de Fourier dans la fibre impaire ( $c f$. plus loin), cette dg-algèbre est isomorphe à l'algèbre des poly-champs de vecteurs formels le long de $\mathfrak{h}^{\perp}$, c'est-à-dire

$$
\mathcal{T}(\mathcal{B}):=S_{\mathcal{B}}(\operatorname{Der}(\mathcal{B})[-1])[1]
$$

où $\mathcal{B}=\mathcal{C}^{\infty}\left(\mathfrak{h}^{\perp}\right) \otimes S\left(\left(\mathfrak{g}^{*} / \mathfrak{h}^{\perp}\right)^{*}\right)=\mathcal{C}^{\infty}\left(\mathfrak{h}^{\perp}\right) \otimes S(\mathfrak{h})$ est l'algèbre des fonctions sur

$$
\widehat{M}:=\mathfrak{h}^{\perp} \oplus \mathfrak{g}^{*} / \mathfrak{h}^{\perp}
$$

polynomiales dans la fibre.

Une fois que l'on a fixé un voisinage tubulaire de $\mathfrak{h}^{\perp}$, on peut identifier ce voisinage avec le fibré normal et on peut identifier $\mathfrak{g}^{*} / \mathfrak{h}^{\perp}$ avec un supplémentaire de $\mathfrak{h}^{\perp}$. On a besoin ici de choisir un supplémentaire de $\mathfrak{h}$.

Les fonctions au voisinage de $\mathfrak{h}^{\perp}$ s'identifient avec leur développement de Taylor partiel dans la direction normale, c'est-à-dire des éléments de $\mathcal{B}$. L'algèbre $\mathcal{T}(\mathcal{B})$ s'identifie alors aux poly-champs de vecteurs formels ${ }^{(7)}$ le long de $\mathfrak{h}^{\perp}$.

Le théorème de Formalité [19] nous dit que la dg-algèbre $\mathcal{T}(\mathcal{A})$ est $L_{\infty}$ quasi-isomorphe à la dg-algèbre $\mathcal{D}_{\text {poly }}(\mathcal{A})$. En combinant la transformée de Fourier et le théorème de Formalité dans le cas des super-espaces, Cattaneo-Felder démontrent le théorème suivant.

(7) On entend par poly-champs de vecteurs dans un voisinage formel de $\mathfrak{h}^{\perp}$ une complétion des $\mathcal{V}\left(\mathfrak{g}^{*}\right) / I_{\mathfrak{h}} \perp$, où $\mathcal{V}\left(\mathfrak{g}^{*}\right)$ désigne les poly-champs de vecteurs sur $\mathfrak{g}^{*}$ et $I_{\mathfrak{h} \perp}$ désigne les poly-champs avec développement de Taylor nul sur $\mathfrak{h}^{\perp}$.

$4^{\mathrm{e}}$ SÉRIE - TOME $41-2008-\mathrm{N}^{\mathrm{o}} 5$ 
Théorème (Cattaneo-Felder [8]). - La dg-algèbre des poly-champs de vecteurs formels le long de $\mathfrak{h}^{\perp}$ est $L_{\infty}$ quasi-isomorphe à la dg-algèbre $\mathcal{D}_{\text {poly }}(\mathcal{A})$.

Si on dispose d'une solution de l'équation de Maurer-Cartan sur $\mathfrak{g}^{*}$, alors on disposera d'une solution de Maurer-Cartan sur $M$ (par transformée de Fourier) puis d'une solution de Maurer-Cartan dans $\mathcal{D}_{\text {poly }}(\mathcal{A})$, c'est-à-dire en général ( $c f$. plus loin) d'une $A_{\infty}$-structure $\operatorname{sur} \mathcal{A}$.

Cas des paires symétriques. - Dans le cas des paires symétriques, le supplémentaire $\mathfrak{p}$ étant canonique, on dispose des équations suivantes.

Notons $\left(K_{i}\right)_{i}$ une base de $\mathfrak{k},\left(P_{j}\right)_{j}$ une base de $\mathfrak{p}$. On note $\left(K_{i}^{*}\right)_{i},\left(P_{j}^{*}\right)_{j}$ les bases duales. On identifie $\mathfrak{k}^{\perp}$ et $\mathfrak{p}^{*}$. On identifie de même $\mathfrak{g}^{*} / \mathfrak{k}^{\perp}=\mathfrak{k}^{*}$ et $\mathfrak{p}^{\perp}$. La super-variété étant

$$
M:=\mathfrak{k}^{\perp} \oplus \Pi \mathfrak{k}=\mathfrak{p}^{*} \oplus \Pi \mathfrak{k},
$$

on aura

$$
\widehat{M}:=\mathfrak{k}^{\perp} \oplus \mathfrak{g}^{*} / \mathfrak{k}^{\perp}=\mathfrak{p}^{*} \oplus \mathfrak{k}^{*} .
$$

On notera $\theta_{i}:=\Pi K_{i}^{*}$ les fonctions de coordonnées sur $\Pi \mathfrak{k}$. La dérivée dans la direction $K_{i}^{*}$ sera notée $\partial_{K_{i}^{*}}$; c'est un champ de vecteurs constant sur $\widehat{M}$. De même la dérivée dans la direction $\Pi K_{i}$ sera notée $\partial_{\Pi K_{i}}$; c'est un champ de vecteurs constant sur $M$.

La transformée de Fourier (avec changement de parité) change la dérivée $\partial_{K_{i}^{*}}$ (c'est un champ de degré impair de $\mathcal{T}(\mathcal{B})$ ) en la fonction $\theta_{i}=\Pi K_{i}^{*}$ (c'est une variable impaire de $\mathcal{A}$ ). De même $K_{i}$ fonction de coordonnée sur $\widehat{M}$ (variable paire de $\mathcal{B}$ ) est changée en $\partial_{\Pi K_{i}}$ (variable paire de $\mathcal{T}(\mathcal{A})$ ).

Le bi-vecteur de Poisson sur $\mathfrak{g}^{*}$ associé à la structure de Poisson s'écrit concrètement ${ }^{(8)}$ :

$$
\pi=\left[K_{i}, K_{j}\right] \partial_{K_{i}^{*}} \wedge \partial_{K_{j}^{*}}+\left[P_{i}, P_{j}\right] \partial_{P_{i}^{*}} \wedge \partial_{P_{j}^{*}}+2\left[K_{i}, P_{j}\right] \partial_{K_{i}^{*}} \wedge \partial_{P_{j}^{*}}
$$

Sa transformée de Fourier partielle, notée $\widehat{\pi}$, est somme d'un champ de vecteurs et d'un 3 -vecteur sur la variété $\mathfrak{k}^{\perp} \oplus \Pi \mathfrak{k}$ :

$$
\hat{\pi}=\theta_{i} \theta_{j} \partial_{\Pi\left[K_{i}, K_{j}\right]}+2\left[K_{i}, P_{j}\right] \theta_{i} \partial_{P_{j}^{*}}+\partial_{P_{i}^{*}} \wedge \partial_{P_{j}^{*}} \wedge \partial_{\Pi\left[P_{i}, P_{j}\right]} .
$$

Comme on le constate, la variété $\mathfrak{k}^{\perp} \oplus \Pi \mathfrak{k}$ ne porte pas de structure de Poisson (car $\hat{\pi}$ n'est pas un 2-vecteur), mais une structure vérifiant l'équation de Maurer-Cartan et qui est homogène si on tient compte de tous les degrés impairs dans $\mathcal{T}(\mathcal{A})$, c'est-à-dire des variables $\theta_{i}$ et $\partial_{P_{i}^{*}}$.

Remarque 1. - La partie 1-champ de $\hat{\pi}$ est clairement associée à la paire symétrique dégénérée (abélianisée, i.e. on a $[\mathfrak{p}, \mathfrak{p}]=0$ ) produit semi-direct de $\mathfrak{k}$ et $\mathfrak{p}: \mathfrak{k} \ltimes \mathfrak{p}$. Ce 1-champ est de carré nul. Compte tenu de la graduation, on peut voir la paire symétrique comme une déformation de la partie abélianisée. Si on considérait la structure déformée formelle $[x, y]=t^{2}[x, y]$ pour $x \in \mathfrak{p}$ et $y \in \mathfrak{p}$ (les autres crochets restant inchangés) on trouverait

$$
\hat{\pi}_{t}=\theta_{i} \theta_{j} \partial_{\Pi\left[K_{i}, K_{j}\right]}+2\left[K_{i}, P_{j}\right] \theta_{i} \partial_{P_{j}^{*}}+t^{2} \partial_{P_{i}^{*}} \wedge \partial_{P_{j}^{*}} \wedge \partial_{\Pi\left[P_{i}, P_{j}\right]} .
$$

Pour $t=0$, on retrouve la paire symétrique abélianisée.

(8) On utilise la convention $\partial_{1} \wedge \cdots \wedge \partial_{n}=\frac{1}{n !} \sum_{\sigma \in S_{n}} \partial_{\sigma(1)} \otimes \cdots \otimes \partial_{\sigma(n)}$. Les indices répétés sont sommés. 
Remarque 2. - L'utilisation de la transformée de Fourier impaire est bien connue pour les algèbres Lie; on considère $\Pi \mathfrak{g}$ muni du 1-champ impair quadratique

$$
Q=\xi_{i} \xi_{j} \partial_{\Pi\left[e_{i}, e_{j}\right]} .
$$

Il vérifie $Q^{2}=\frac{1}{2}[Q, Q]=0$. On peut alors appliquer la quantification de Kontsevich dans ce contexte. Les formules de Kontsevich sont plus simples et on peut par exemple décrire de manière plus naturelle le $L_{\infty}$ quasi-isomorphisme tangent qui, par l'argument d'homotopie, réalisera dans le cas des algèbres de Lie, l'isomorphisme de Duflo généralisé

$$
H(\mathfrak{g}, S(\mathfrak{g})) \underset{\text { algèbre }}{\sim} H(\mathfrak{g}, U(\mathfrak{g})) .
$$

Cette méthode a été expérimentée par Shoikhet [31] (voir aussi [24]).

Remarque 3. - Le $L_{\infty}$ morphisme agit aussi sur les poly-champs dont la restriction à $\mathfrak{h}^{\perp}$ est nulle sur $\bigwedge \mathfrak{h}$, c'est-à-dire les poly-champs de vecteurs dont la restriction est nulle sur la puissance extérieure du fibré conormal; ce sont les poly-vecteurs dits relatifs; par exemple le bi-vecteur de Poisson $\pi$ vérifie cette propriété (voir [7]).

\subsection{Construction en termes de diagrammes de Feynman}

La formule proposée est semblable à celle de Kontsevich [19] pour $\mathbb{R}^{n}$. Elle fait intervenir des graphes numérotés, des coefficients obtenus par intégration de formes différentielles sur des variétés de configurations dans le demi-plan de Poincaré et des opérateurs polydifférentiels associés à ces graphes ${ }^{(9)}$. Pour simplifier la lecture de cet article on rappelle brièvement les ingrédients de la construction générale.

Variétés de configurations. - On note $C_{n, m}$ l'espace des configurations de $n$ points distincts dans $\mathcal{H}$ le demi-plan de Poincaré (points de première espèce ou points aériens) et $m$ points distincts sur la droite réelle (points de seconde espèce ou points terrestres), modulo l'action du groupe $a z+b$ (pour $a \in \mathbb{R}^{+*}, b \in \mathbb{R}$ ). Dans son article [19], Kontsevich construit des compactifications de ces variétés notées $\bar{C}_{n, m}$. Ce sont des variétés à coins de dimension $2 n-2+m$. Ces variétés ne sont pas connexes pour $m \geq 2$. On notera $\bar{C}_{n, m}^{+}$la composante qui contient les configurations où les points terrestres sont ordonnés dans l'ordre croissant (i.e. on a $\overline{1}<\overline{2}<\cdots<\bar{m}$ ).

Fonctions d'angle à deux couleurs. - Les graphes de Kontsevich vont être colorés en fonction de la variable de dérivation associée dans l'opérateur; on aura donc besoin d'une fonction d'angle qui dépend de deux couleurs notées + et - . Dans les figures, la couleur + sera représentée en traits pleins et la couleur - sera représentée en traits pointillés.

DÉfinition 1. - On définit les deux fonctions d'angles de $C_{2,0}$ dans $\mathbb{S}^{1}$

$$
\left\{\begin{array}{l}
\phi_{+}(p, q)=\underset{p}{\bullet} \longrightarrow \underset{q}{\bullet}=\arg (p-q)+\arg (p-\bar{q}) \\
\phi_{-}(p, q)=\dot{\bullet}_{p}-\rightarrow \underset{q}{\bullet}=\arg (p-q)-\arg (p-\bar{q}) .
\end{array}\right.
$$

(9) On pourra consulter les références [4], [9] pour une description détaillée de la construction de Kontsevich.

$4^{\text {e }}$ SÉRIE - TOME $41-2008-\mathrm{N}^{\mathrm{o}} 5$ 
Ces fonctions d'angle s'étendent à la compactification $\bar{C}_{2,0}$. La fonction d'angle $\phi_{+}$sera associée aux variables tangentes (i.e. dans $\mathfrak{h}^{\perp}$ ) tandis que la fonction d'angle $\phi_{-}$sera associée aux variables normales (i.e. dans $\left.\mathfrak{g}^{*} / \mathfrak{h}^{\perp}=\mathfrak{h}^{*}\right)^{(10)}$. Remarquons que l'on a

$$
\mathrm{d} \phi_{-}(p, q)=\mathrm{d} \phi_{+}(q, p) .
$$

Le fonction d'angle $\phi_{+}$est celle définie par Kontsevich.

Graphes et opérateurs différentiels associés. - Les graphes qui vont intervenir dans la définition du $L_{\infty}$ quasi-isomorphisme sont analogues à ceux de Kontsevich [19] avec la différence essentielle suivante, les arêtes sont colorées par nos deux couleurs + (dans les dessins $\longrightarrow$ ) et - (dans les dessins $\rightarrow \rightarrow$ ).

Soit $\Gamma$ un graphe (quiver) avec $n$ sommets de première espèce (aériens) numérotés $1,2, \ldots, n$ et $m$ sommets de seconde espèce (terrestres) numérotés $\overline{1}, \ldots, \bar{m}$.

Par construction $\Gamma$ n'a pas de boucles ${ }^{(11)}$, ni d'arêtes doubles (même source, même but, même couleur). Les arêtes, issues des sommets de seconde espèce, sont colorées par la couleur - et ne reçoivent que des arêtes colorées par la couleur + . Par ailleurs un certain nombre d'arêtes, portant la couleur -, n'ont pas de but. On dira qu'elles vont à l'infini ( $c f$. Fig. 1).

Notons $k_{1}, \ldots k_{n}$ le nombre d'arêtes sortant des sommets de première espèce et $k_{\overline{1}}, \ldots, k_{\bar{m}}$ le nombre d'arêtes sortant des sommets de seconde espèce. Soient $\xi_{1}, \ldots, \xi_{n}$ des polyvecteurs, avec $k_{i}$ le degré de $\xi_{i}$ et $f_{\overline{1}}, \ldots, f_{\bar{m}}$ des fonctions avec $f_{\bar{j}} \in \mathcal{C}^{\infty}\left(\mathfrak{h}^{\perp}\right) \otimes \bigwedge^{k_{\bar{j}}} \mathfrak{h}^{*}$. En plaçant au sommet aérien $i$ le poly-vecteur $\xi_{i}$ et au sommet terrestre $\bar{j}$ la fonction $f_{\bar{j}}$, on définit après restriction à $\mathfrak{h}^{\perp}$, une fonction (poly-champ)

$$
B_{\Gamma}\left(\xi_{1}, \ldots, \xi_{n}\right)\left(f_{\overline{1}}, \cdots, f_{\bar{m}}\right) \in \mathcal{C}^{\infty}\left(\mathfrak{h}^{\perp}\right) \otimes \bigwedge^{p} \mathfrak{h}^{*}
$$

où $p$ désigne le nombre d'arêtes qui partent à l'infini. La règle complémentaire est la suivante : si l'arête porte la couleur + , on ne dérive que selon les variables tangentes (i.e. dans $\mathfrak{h}^{\perp}$ ) et si la couleur est - , on ne dérive que selon les variables normales (i.e. dans $\mathfrak{h}^{*}$ ). On définit ainsi un opérateur poly-différentiel $B_{\Gamma}\left(\xi_{1}, \ldots, \xi_{n}\right)$. Remarquons que les arêtes, qui partent à l'infini, contribuent à la définition de cet opérateur.

Coefficients. - Soit $\Gamma$ un graphe coloré avec $n$ sommets de première espèce et $m$ sommets de seconde espèce. On dessine le graphe dans $C_{n, m}^{+}$. Toute arête colorée $e$, qui ne part pas à l'infini, définit par restriction une fonction d'angle notée $\phi_{e}$ sur la variété $\bar{C}_{n, m}^{+}$. Si la couleur est $\varepsilon \in\{+,-\}$, on choisit la fonction d'angle $\phi_{\varepsilon}$. On note $E_{\Gamma}$ l'ensemble des arêtes du graphe $\Gamma$ qui ne partent pas à l'infini. Le produit ordonné $\Omega_{\Gamma}=\bigwedge_{e \in E_{\Gamma}} \mathrm{d} \phi_{e}$ est donc une $\sharp E_{\Gamma}$-forme régulière $\operatorname{sur} \bar{C}_{n, m}^{+}$, variété compacte de dimension $2 n+m-2$.

DÉfINITION 2. - Le poids, associé à un graphe coloré $\Gamma$, est défini par l'intégrale

$$
w_{\Gamma}=\frac{1}{(2 \pi)^{\sharp E_{\Gamma}}} \int_{\bar{C}_{n, m}^{+}} \Omega_{\Gamma} .
$$

(10) On a besoin ici de faire un choix d'un supplémentaire de $\mathfrak{h}$.

(11) Arête dont la source et le but sont identiques. 
Ce coefficient est nul si $\sharp E_{\Gamma} \neq 2 n+m-2$. Remarquons que les arêtes qui partent (resp. qui arrivent) de l'axe réel portent la couleur - (resp. +); par conséquent les différentielles des fonctions d'angle associées ne sont pas nulles.

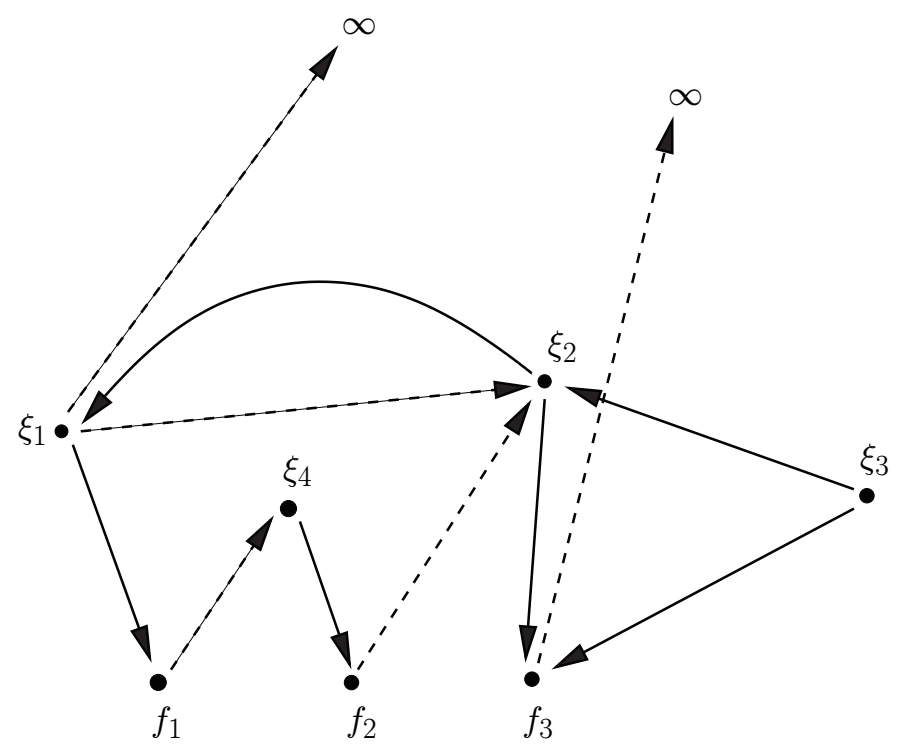

FIGURE 1. Graphe type intervenant dans le calcul de $U_{4}$

Construction du $L_{\infty}$-quasi-isomorphisme. - Pour $n \geq 0$, on note

$$
U_{n}\left(\xi_{1}, \ldots, \xi_{n}\right):=\sum_{\Gamma} w_{\Gamma} B_{\Gamma}\left(\xi_{1}, \ldots, \xi_{n}\right),
$$

où la somme porte sur tous les graphes avec $n$ sommets aériens sur lesquels on a placé les polyvecteurs $\xi_{1}, \ldots, \xi_{n}$ et un nombre quelconque de sommets terrestres.

L'opérateur $U_{n}\left(\xi_{1}, \ldots, \xi_{n}\right)$ n'est pas homogène pour la graduation de Hochschild, car la diminution du nombre d'arguments peut être compensée par l'augmentation du degré en $\mathfrak{h}^{*}$. Pour $n=0$, nécessairement $m$ vaut 2 et on retrouve la multiplication dans $\mathcal{C}^{\infty}\left(\mathfrak{h}^{\perp}\right) \otimes \wedge \mathfrak{h}^{*}$.

THÉORÈme 1.1 (Cattaneo-Felder). - La somme $U=\sum_{n \geq 1} \frac{1}{n !} U_{n}$ définit un $L_{\infty}$ quasiisomorphisme de la dg-algèbre des poly-champs de vecteurs formels dans un voisinage de $\mathfrak{h}^{\perp}$ dans $\mathcal{D}_{\text {poly }}\left(\mathcal{C}^{\infty}\left(\mathfrak{h}^{\perp}\right) \otimes \bigwedge \mathfrak{h}^{*}\right)$.

Comme dans le cas classique, c'est la formule de Stokes qui fournit l'équation du $L_{\infty}$ quasi-isomorphisme.

$4^{\mathrm{e}}$ SÉRIE - TOME $41-2008-\mathrm{N}^{\mathrm{o}} 5$ 


\subsection{Cas des bi-vecteurs de Poisson et cas des bi-vecteurs de Poisson linéaires}

Cas des bi-vecteurs de Poisson. - Lorsqu'on applique la construction précédente dans le cas où les poly-vecteurs $\xi_{i}$ sont égaux à un 2-vecteur de Poisson $\pi$, on trouve un opérateur polydifférentiel formel non homogène du complexe de Hochschild. Mais cet opérateur est homogène de degré 1 si l'on tient compte des degrés impairs.

Dans le cas des variétés de Poisson, étudié dans [19], l'opérateur est de degré 1 dans le complexe de Hochschild, c'est donc un produit associatif. Ici la situation est plus compliquée. Comme la graduation tient compte du degré dans les variables impaires, la structure obtenue est en fait une $A_{\infty}$-structure sur l'espace $\mathcal{A}=\mathcal{C}^{\infty}\left(\mathfrak{h}^{\perp}\right) \otimes \wedge \mathfrak{h}^{*}$ avec premier terme non nul $a$ priori, c'est-à-dire une structure ${ }^{(12)}$

$$
\mu=\mu_{-1}+\mu_{0}+\mu_{1}+\mu_{2}+\ldots
$$

vérifiant $\frac{1}{2}[\mu, \mu]_{G H}=0$ ou en terme de bar-construction $\bar{\mu} \circ \bar{\mu}=0$, où $\bar{\mu}$ désigne la codérivation, associée à $\mu$, de l'algèbre tensorielle.

A priori, il existe donc des composantes en tout degré de Hochschild (mais ces composantes sont homogènes de degré 1 si l'on tient compte de la graduation $\wedge \mathfrak{h}^{*}$ ); on a donc en général :

- Une composante $\mu_{-1}$, que l'on note aussi $F_{\pi}$ (degré -1 dans le complexe de Hochschild, c'est-à-dire un élément de $\mathcal{A}$ ) : c'est une sorte de courbure.

- Une composante $\mu_{0}$, qui ne prend qu'un argument que l'on note aussi $A_{\pi}$ (degré 0 dans le complexe de Hochschild, c'est-à-dire un opérateur différentiel formel sur $\mathcal{A}$ ) : c'est presque une différentielle.

- Une composante $\mu_{1}$, qui prend deux arguments que l'on note aussi $B_{\pi}$ (degré 1 dans le complexe de Hochschild, c'est-à-dire un opérateur bi-différentiel formel sur $\mathcal{A}$ ) : c'est presque un produit (associatif).

- etc.

On écrit alors $\mu=F_{\pi}+A_{\pi}+B_{\pi}+B_{2}+\ldots$

Faisons l'inventaire des arêtes. - Un graphe avec $n$ sommets aériens et $m$ points terrestres produit (dans sa partie aérienne) $2 n$ arêtes pour une dimension de variété de configurations $2 n+m-2$. Il faut donc disposer, sur l'axe réel, $m$ poly-vecteurs fournissant au moins $m-2$ arêtes. $\mathrm{Si}$ au total on a plus de $2 n+m-2$ arêtes, alors il faut faire sortir à l'infini le nombre adéquat d'arêtes, colorées par $\mathfrak{h}^{*}$.

Dans le cas $m=0$, il faut donc 2 arêtes sortantes, donc $F_{\pi}$ est un 2-vecteur. On remarquera que $F_{\pi}$ est alors de degré $1=-1+2$. C'est une sorte de courbure.

Toutefois si $F_{\pi}=0$, alors $A_{\pi}$ sera une différentielle vérifiant $\left[A_{\pi}, B_{\pi}\right]_{G H}=0$ et on aura l'équation ${ }^{(13)}$

$$
\left[B_{\pi}, B_{\pi}\right]_{G H}+2\left[B_{2}, A_{\pi}\right]_{G H}=0
$$

où $B_{2}$ désigne la composante de degré 2 (qui prend 3 arguments). En conséquence $B_{\pi}$ sera un produit associatif dans l'espace de cohomologie défini par $A_{\pi}$.

(12) On note $[,]_{G H}$ le crochet de Gerstenhaber.

(13) On fera attention que l'on n'a pas $\left[B_{2}, A_{\pi}\right]_{G H}=-\left[A_{\pi}, B_{2}\right]_{G H}$, car il faut tenir compte de la graduation des coefficients; on a donc $\left[B_{2}, A_{\pi}\right]_{G H}=\left[A_{\pi}, B_{2}\right]_{G H}$. 
Cas linéaire. - On se place dans le cas où $\pi$, le bi-vecteur de Poisson placé aux sommets aériens, est égal à la moitié ${ }^{(14)}$ du bivecteur de Poisson linéaire associé à l'algèbre de Lie $\mathfrak{g}$. On suppose toujours que $\mathfrak{h}$ est une sous-algèbre. Toutes les constructions se restreignent à l'algèbre des fonctions polynomiales notée $C_{\text {poly }}\left(\mathfrak{h}^{\perp}\right) \otimes \wedge \mathfrak{h}^{*}$.

Nullité de la courbure

Lemme 1. - Dans le cas linéaire, pour toute sous-algèbre $\mathfrak{h}$, on a $F_{\pi}=0$.

Démonstration. - On doit avoir $2 n-2$ arêtes dans le graphe mais on ne dispose que de $n$ sommets à dériver. On doit donc avoir par linéarité de $\pi, 2 n-2 \leq n$, c'est-à-dire $n=1$ ou $n=2$.

- Si $n=1$, la restriction de $\pi$ à $\bigwedge^{2} \mathfrak{h}$ est nulle si et seulement si h est une sous-algèbre (on retrouve la condition de co-isotropie de la variété $\left.C=\mathfrak{h}^{\perp}\right)$.

- Si $n=2$, les graphes qui interviennent sont comme dans (1.12) (dessins de gauche) car le 2-vecteur est linéaire. Dans ce cas le coefficient est nul, car on a une arête double ou deux arêtes qui se suivent ${ }^{(15)}$.

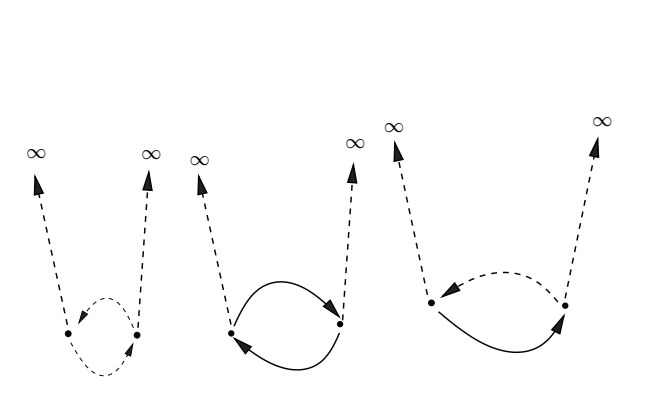

Graphes pour $F_{\pi}$

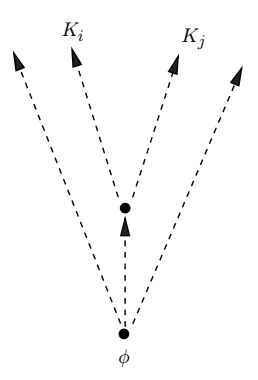

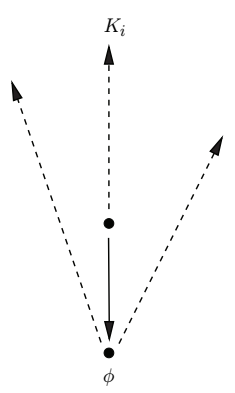

Graphes pour $A_{\pi}$

Déformation de la différentielle de Cartan-Eilenberg. - Il existe une graduation liée au nombre de sommets aériens dans les graphes de Kontsevich. On note $\varepsilon$ le paramètre de graduation; cela revient à changer $\pi$ en $\varepsilon \pi$ dans les formules.

Lemme 2. - Le terme $A_{\pi}$ est une différentielle. On a $A_{\pi}=\varepsilon d_{C E}+o(\varepsilon)$, où $d_{C E}$ désigne la différentielle de Cartan-Eilenberg de $\mathfrak{h}$ agissant dans le $\mathfrak{h}$-module $S(\mathfrak{g}) / S(\mathfrak{g}) \cdot \mathfrak{h}=S(\mathfrak{g} / \mathfrak{h})=$ $\mathcal{C}_{\text {poly }}\left(\mathfrak{h}^{\perp}\right)$.

Démonstration. - Pour $n=1$, les seuls graphes qui interviennent sont comme dans (1.12) (les deux dessins de droite). Pour $\phi \in C_{\text {poly }}\left(\mathfrak{h}^{\perp}\right) \otimes \wedge \mathfrak{h}^{*}$ de degré $q-1$ les graphes dessinés contribuent comme

$$
\sum_{i<j}(-1)^{i+j} \phi\left(\left[K_{i}, K_{j}\right], K_{1}, \ldots, \hat{K}_{i}, \ldots, \hat{K}_{j}, \ldots, K_{q}\right)
$$

\footnotetext{
(14) Les graphes « géométriques » apparaissent de nombreuses fois à cause de la numérotation, ce qui explique cette normalisation.

(15) Remarquons que pour $n=1$ ou $n=2$ les contributions sont toujours nulles dans $F_{\pi}$, même si $\pi$ n'est pas linéaire.

$4^{\text {e }}$ SÉRIE - TOME $41-2008-\mathrm{N}^{\mathrm{o}} 5$
} 
et

$$
\sum_{i}(-1)^{i+1} \operatorname{ad} K_{i}\left(\phi\left(K_{1}, \ldots, \hat{K}_{i}, \ldots, K_{q}\right)\right) .
$$

On reconnaît la différentielle de Cartan-Eilenberg.

Définition 3. - On note $H_{\varepsilon}^{\bullet}\left(\mathfrak{h}^{\perp}\right)$ l'espace de cohomologie de réduction formelle pour la différentielle $A_{\pi}$ agissant dans $\mathcal{C}_{\text {poly }}\left(\mathfrak{h}^{\perp}\right)[[\varepsilon]] \otimes \wedge \mathfrak{h}^{*}$. L'espace $H_{\varepsilon}^{0}\left(\mathfrak{h}^{\perp}\right)$ sera appelé l'espace de réduction.

Pour $\phi \in H_{\varepsilon}^{\bullet}\left(\mathfrak{h}^{\perp}\right)$, on a $\phi=\phi_{o}+\varepsilon \phi_{1}+\varepsilon^{2} \phi_{2}+\cdots$. Le premier terme $\phi_{o}$ est dans l'espace de cohomologie relative

$$
H_{C E}^{\bullet}(\mathfrak{h}, S(\mathfrak{g} / \mathfrak{h}))
$$

car on a $A_{\pi}=\varepsilon d_{C E}+o(\varepsilon)$. Toutefois, on ne peut pas affirmer que $H_{\varepsilon}^{\bullet}\left(\mathfrak{h}^{\perp}\right)$ est une déformation de $H^{\bullet}(\mathfrak{h}, S(\mathfrak{g} / \mathfrak{h}))$, car l'application $\phi \mapsto \phi_{o}$ n'est pas a priori surjective.

En particulier pour $f \in H_{\varepsilon}^{0}\left(\mathfrak{h}^{\perp}\right)$, c'est-à-dire une fonction $f=f_{0}+\varepsilon f_{1}+\varepsilon^{2} f_{2}+\ldots$ dans $\mathcal{C}_{\text {poly }}\left(\mathfrak{h}^{\perp}\right)[[\varepsilon]]$ solution de $A_{\pi}(f)=0$, on aura $f_{0} \in S(\mathfrak{g} / \mathfrak{h})^{\mathfrak{h}}$. L'application symbole, $f \mapsto f_{0}$ n'est pas surjective a priori et on ne pourra pas considérer $H_{\varepsilon}^{0}\left(\mathfrak{h}^{\perp}\right)$ comme une déformation de l'espace $S(\mathfrak{g} / \mathfrak{h})^{\mathfrak{h}}$. C'est toutefois le cas pour les paires symétriques et plus généralement lorsque $\mathfrak{h}$ admet un supplémentaire $\mathfrak{h}$-invariant ( $c f$. Proposition 4$)$.

Structure associative en cohomologie. - La courbure étant nulle dans notre situation linéaire, l'équation générale

$$
\left[B_{\pi}, B_{\pi}\right]_{G H}+2\left[B_{2}, A_{\pi}\right]_{G H}=0
$$

montre que $B_{\pi}$ définit un produit associatif en cohomologie. Cette formule résulte de la formule de Stokes avec 3 points sur l'axe réel, il faut tenir compte des strates de bord qui contiennent tout le chemin : cette strate fournit un co-bord pour la différentielle $A_{\pi}$. Donc ce n'est qu'en cohomologie que ces formules fournissent un produit associatif. On a donc la proposition suivante ${ }^{(16)}$.

Proposition 1. - Dans le cas linéaire, $B_{\pi}$ définit un produit associatif dans $H_{\varepsilon}^{\bullet}\left(\mathfrak{h}^{\perp}\right)$.

\subsection{Dépendance par rapport au choix du supplémentaire}

On examine, dans le cas linéaire, la dépendance du produit associatif de la Proposition 1, par rapport au choix du supplémentaire.

(16) $\mathrm{Si} \pi$ est un bi-vecteur quelconque, cette proposition est encore vraie dès que l'on a $F_{\pi}=0$ (cf. [7]) 
Action du champ de vecteurs de déformation. - On fixe une base $\left(K_{i}\right)_{i}$ de $\mathfrak{h}$. Fixons un supplémentaire $\mathfrak{q}_{0}$ de $\mathfrak{h}$. On fixe une base $\left(P_{a}\right)_{a}$ de $\mathfrak{q}_{0}$, ce qui permet de considérer $\left(P_{a}^{*}\right)_{a}$ base de $\mathfrak{h}^{\perp}$.

Faisons le choix d'un autre supplémentaire $\mathfrak{q}_{1}$ dont on fixe une base $\left(Q_{a}\right)_{a}$, ce qui permet de considérer $\left(Q_{a}^{*}\right)_{a}$. On suppose que l'on a $P_{a}^{*}=Q_{a}^{*}$, i.e. on a $P_{a}-Q_{a} \in \mathfrak{h}$.

Notons $\mu_{\mathfrak{q}_{0}}$ et $\mu_{\mathfrak{q}_{1}}$ les structures $A_{\infty}$ construites pour ces choix de supplémentaires. On notera dorénavant $\mathcal{A}$ l'algèbre des fonctions polynomiales sur la super-variété $\mathfrak{h}^{\perp} \oplus \Pi \mathfrak{h}$ :

$$
\mathcal{A}:=\mathcal{C}_{\text {poly }}\left(\mathfrak{h}^{\perp}\right) \otimes \bigwedge\left(\mathfrak{g}^{*} / \mathfrak{h}^{\perp}\right) \simeq \mathcal{C}_{\text {poly }}\left(\mathfrak{h}^{\perp}\right) \otimes \bigwedge \mathfrak{h}^{*}
$$

Changer de supplémentaire revient à faire un changement de coordonnées linéaires sur $\mathfrak{g}$. La matrice de changement de bases de $\left(K_{i}, P_{a}\right)$ vers $\left(K_{i}, Q_{a}\right)$ a la forme suivante :

$$
\mathbb{M}=\left(\begin{array}{cc}
I d & \mathbb{D} \\
0 & I d
\end{array}\right)
$$

Notons $\mathbb{D}=\left[V_{1}, \ldots, V_{p}\right]$ les colonnes de la matrice $\mathbb{D}$ avec $V_{i} \in \mathfrak{h}$.

Par transformée de Fourier partielle, $\widehat{\partial_{K_{i}^{*}}}$ correspond à la fonction sur $\Pi \mathfrak{h}$ notée $\theta_{K_{i}^{*}}$, $\widehat{K}_{i}$ correspond à dérivée $\partial_{\Pi K_{i}}$ sur $\Pi \mathfrak{h}$. Les variables $P_{a}$ et $\partial_{P_{a}^{*}}$ ne sont pas changées.

La variété $\mathfrak{h}^{\perp} \oplus \Pi \mathfrak{h}$ est intrinsèque et ne dépend pas du choix de la décomposition. Les fonctions $P_{a}$ et $Q_{a}$ sont égales comme fonctions sur $\mathfrak{h}^{\perp}$. Les fonctions $\theta_{K_{i}^{*}}$ (pour les deux décompositions) sont égales sur $\Pi \mathfrak{h}$.

Les bi-vecteurs de Poisson pour les deux décompositions s'écrivent respectivement ${ }^{(17)}$ :

$$
\begin{aligned}
\pi & =\left[K_{i}, K_{j}\right] \partial_{K_{i}^{*}} \wedge \partial_{K_{j}^{*}}+2\left[K_{i}, P_{a}\right] \partial_{K_{i}^{*}} \wedge \partial_{P_{a}^{*}}+\left[P_{a}, P_{b}\right] \partial_{P_{a}^{*}} \wedge \partial_{P_{b}^{*}} \\
\pi_{1} & =\left[K_{i}, K_{j}\right] \partial_{K_{i}^{*}} \wedge \partial_{K_{j}^{*}}+2\left[K_{i}, Q_{a}\right] \partial_{K_{i}^{*}} \wedge \partial_{Q_{a}^{*}}+\left[Q_{a}, Q_{b}\right] \partial_{Q_{a}^{*}} \wedge \partial_{Q_{b}^{*}}
\end{aligned}
$$

Par transformée de Fourier partielle notée $\mathcal{F}$, on a :

$$
\begin{aligned}
\widehat{\pi} & =\partial_{\Pi\left[K_{i}, K_{j}\right]} \theta_{K_{i}^{*}} \theta_{K_{j}^{*}}+2 \mathcal{F}\left(\left[K_{i}, P_{a}\right]\right) \theta_{K_{i}^{*}} \partial_{P_{a}^{*}}+\mathcal{F}\left(\left[P_{a}, P_{b}\right]\right) \partial_{P_{a}^{*}} \wedge \partial_{P_{b}^{*}} \\
\widehat{\pi_{1}} & =\partial_{\Pi\left[K_{i}, K_{j}\right]} \theta_{K_{i}^{*}} \theta_{K_{j}^{*}}+2 \mathcal{F}\left(\left[K_{i}, Q_{a}\right]\right) \theta_{K_{i}^{*}} \partial_{Q_{a}^{*}}+\mathcal{F}\left(\left[Q_{a}, Q_{b}\right]\right) \partial_{Q_{a}^{*}} \wedge \partial_{Q_{b}^{*}} .
\end{aligned}
$$

Comme on a $\partial_{Q_{a}^{*}}=\partial_{P_{a}^{*}}$, le changement de supplémentaire n'opère finalement que sur les coefficients. Soient $\left(e_{i}\right)_{i}$ une base de $\mathfrak{g}$ adaptée à la première décomposition et $\left(e_{i}^{*}\right)_{i}$ sa base duale. Considérons le bivecteur :

$$
\pi_{\mathbb{M}}:=\mathbb{M}^{-1}\left[\mathbb{M} e_{i}, \mathbb{M} e_{j}\right] \partial_{e_{i}^{*}} \wedge \partial_{e_{j}^{*}}
$$

Alors on aura

$$
\widehat{\pi_{\mathbb{M}}}=\widehat{\pi_{1}} .
$$

Lemme 3. - Notons $v=-V_{a} \partial_{P_{a}^{*}}$ le champ de vecteurs sur $\mathfrak{g}^{*}$. On $a[v, v]_{S N}=0^{(18)}$ et

$$
\pi_{\mathbb{M}}=e^{\mathrm{ad} v} \cdot \pi=\pi+[v, \pi]_{S N}+\frac{1}{2}[v,[v, \pi]]_{S N} .
$$

L'action du champ -v sur le bivecteur $\pi$ correspond au changement de supplémentaire.

(17) On convient, comme d'habitude, que les indices répétés sont sommés.

(18) On note $[,]_{S N}$ le crochet de Schouten-Nijenhuis. 
Démonstration. - Vérifions ce fait dans le cas des paires symétriques (mais le résultat reste vrai en général). On a, avec les notations introduites pour la matrice $\mathbb{M}$, les équations suivantes :

$$
\begin{gathered}
\mathbb{M}^{-1}\left[\mathbb{M} K_{i}, \mathbb{M} K_{j}\right]=\left[K_{i}, K_{j}\right] \\
2 \mathbb{M}^{-1}\left[\mathbb{M} K_{i}, \mathbb{M} P_{a}\right]=2 \mathbb{M}^{-1}[K_{i}, P_{a}+\underbrace{\mathbb{D} P_{a}}_{\in \mathfrak{k}}]=2\left[K_{i}, P_{a}\right]-\underbrace{2 \mathbb{D}\left[K_{i}, P_{a}\right]}_{(1)}+\underbrace{2\left[K_{i}, \mathbb{D} P_{a}\right]}_{(2)}
\end{gathered}
$$

$$
\begin{aligned}
\mathbb{M}^{-1}\left[\mathbb{M} P_{a}, \mathbb{M} P_{b}\right]=\mathbb{M}^{-1}\left[P_{a}+\mathbb{D} P_{a}, P_{b}+\mathbb{D} P_{b}\right]= \\
\underbrace{\left[P_{a}, P_{b}\right]}_{\in \mathfrak{k}}+\underbrace{\left[\mathbb{D} P_{a}, \mathbb{D} P_{b}\right]}_{(3)}+\underbrace{\left(\left[\mathbb{D} P_{a}, P_{b}\right]+\left[P_{a}, \mathbb{D} P_{b}\right]\right)}_{\in \mathfrak{k}}-\underbrace{\mathbb{D}(\underbrace{\left(\left[\mathbb{D} P_{a}, P_{b}\right]\right.}_{(4)}+\left[P_{\in \mathfrak{p}}, \mathbb{D} P_{b}\right])}_{\in \mathfrak{p}} \underbrace{\underbrace{}_{\in \mathfrak{p}}}_{(5)}
\end{aligned}
$$

Calculons $[v, \pi]_{S N}=v \bullet \pi+\pi \bullet v$. On a les formules suivantes :

$$
\begin{aligned}
& -v \bullet \pi=2 V_{a}\left\langle P_{a}^{*},\left[K_{i}, P_{b}\right]\right\rangle \partial_{K_{i}^{*}} \wedge \partial_{P_{b}^{*}} \quad=2 \underbrace{\mathbb{D}\left[K_{i}, P_{b}\right]}_{\in \mathfrak{k}} \partial_{K_{i}^{*}} \wedge \partial_{P_{b}^{*}} \\
& -\pi \bullet v=2[\underbrace{\left[V_{a}, K_{i}\right]}_{\in \mathfrak{k}} \partial_{K_{i}^{*}} \wedge \partial_{P_{a}^{*}}+2 \underbrace{\left[V_{a}, P_{b}\right]}_{\in \mathfrak{p}} \partial_{P_{b}^{*}} \wedge \partial_{P_{a}^{*}} \\
& v \bullet(v \bullet \pi)= \\
& 0 \\
& v \bullet(\pi \bullet v)=\quad 2 V_{c}\left\langle P_{c}^{*},\left[V_{a}, P_{b}\right]\right\rangle \partial_{P_{b}^{*}} \wedge \partial_{P_{a}^{*}} \quad=2 \underbrace{\mathbb{D}\left[V_{a}, P_{b}\right]}_{\in \mathfrak{k}} \partial_{P_{b}^{*}} \wedge \partial_{P_{a}^{*}} \\
& (v \bullet \pi) \bullet v=\quad \underbrace{\mathbb{D}\left[K_{i}, P_{b}\right]}_{\in \mathfrak{k}}\left\langle K_{i}^{*}, V_{c}\right\rangle \partial_{P_{b}^{*}} \wedge \partial_{P_{c}^{*}} \quad=2 \underbrace{\mathbb{D}\left[V_{c}, P_{b}\right]}_{\in \mathfrak{k}} \partial_{P_{b}^{*}} \wedge \partial_{P_{c}^{*}} \\
& (\pi \bullet v) \bullet v=\quad 2[\underbrace{\left[V_{a}, K_{i}\right]}_{\in \mathfrak{k}}\left\langle K_{i}^{*}, V_{c}\right\rangle \partial_{P_{a}^{*}} \wedge \partial_{P_{c}^{*}} \quad=2[\underbrace{\left[V_{a}, V_{c}\right]}_{\in \mathfrak{k}} \partial_{P_{a}^{*}} \wedge \partial_{P_{c}^{*}} .
\end{aligned}
$$

On a donc

$$
[v, \pi]_{S N}=\underbrace{-2 \underbrace{\mathbb{D}\left[K_{i}, P_{a}\right]}_{\in \mathfrak{k}} \partial_{K_{i}^{*}} \wedge \partial_{P_{a}^{*}}}_{(1)}+\underbrace{2 \underbrace{\left[K_{i}, \mathbb{D} P_{a}\right]}_{\in \mathfrak{k}} \partial_{K_{i}^{*}} \wedge \partial_{P_{a}^{*}}}_{(2)}+\underbrace{2 \underbrace{\left[\mathbb{D} P_{a}, P_{b}\right]}_{\in \mathfrak{p}} \partial_{P_{a}^{*}} \wedge \partial_{P_{b}^{*}}}_{(4)}
$$

et

$$
\frac{1}{2}[v,[v, \pi]]_{S N}=-\underbrace{-\underbrace{\mathbb{D}\left[\mathbb{D} P_{a}, P_{b}\right]}_{\in \mathfrak{k}} \partial_{P_{a}^{*}} \wedge \partial_{P_{b}^{*}}}_{(5)}-\underbrace{\left[\mathbb{D} P_{a}, \mathbb{D} P_{b}\right]}_{(3)} \partial_{P_{a}^{*}} \wedge \partial_{P_{b}^{*}} .
$$

On retrouve bien les termes de $\pi_{\mathbb{M}}$ des équations (1.17) et (1.18).

Dépendance par rapport au supplémentaire. - Considérons maintenant la transformée de Fourier partielle. L'action infinitésimale est donnée alors par $[\widehat{v}, \widehat{\pi}]_{S N}$. L'expression

$$
\widehat{\pi}+[\widehat{v}, \widehat{\pi}]_{S N}+\frac{1}{2}[\widehat{v},[\widehat{v}, \widehat{\pi}]]_{S N}
$$

représente la transformée de Fourier du bi-vecteur $\pi$ pour le deuxième choix de supplémentaire, exprimée dans les coordonnées intrinsèques de $\mathfrak{h}^{\perp} \oplus \Pi \mathfrak{h}$. 
La transformée de Fourier partielle $\widehat{v}$ du champ $v$, s'écrit $-\partial_{\Pi V_{a}} \partial_{P_{a}^{*}}$. C'est donc un 2 -vecteur à coefficients constants, qui vérifie aussi l'équation $[\widehat{v}, \widehat{v}]_{S N}=0$.

Appliquons le $L_{\infty}$ quasi-isomorphisme de Kontsevich noté ici $U$. On note $m$ la multiplication dans $\mathcal{C}_{\text {poly }}\left(\mathfrak{h}^{\perp}\right)[[\varepsilon]] \otimes \wedge \mathfrak{h}^{*}$. On définit $\pi_{t} \operatorname{par}^{(19)}$

$$
\left.\pi_{t}:=e^{t \mathrm{ad} v} \cdot \pi=\pi+t[v, \pi]+\frac{t^{2}}{2}[v,[v, \pi]]\right] .
$$

On a $\pi_{t=0}=\pi$ et $\pi_{t=1}=\pi_{1}$. On désigne par $\mu_{t}$ la structure $A_{\infty}$ correspondante :

$$
\mu_{t}:=m+U\left(e^{\widehat{\pi_{t}}}-1\right)=m+\sum_{n \geq 1} \frac{\varepsilon^{n}}{n !} U_{n}\left(\widehat{\pi_{t}}, \ldots, \widehat{\pi_{t}}\right) .
$$

Il vient en conséquence :

$$
\begin{aligned}
\mu_{\mathfrak{q}_{1}} & =\mu_{t=1}=m+U\left(e^{\widehat{\pi}+[\widehat{v}, \widehat{\pi}]+\frac{1}{2}[\widehat{v},[\widehat{v}, \widehat{\pi}]]}-1\right) \\
& =m+\sum_{n \geq 1} \frac{\varepsilon^{n}}{n !} U_{n}\left(\widehat{\pi}+[\widehat{v}, \widehat{\pi}]+\frac{1}{2}[\widehat{v},[\widehat{v}, \widehat{\pi}]], \ldots, \widehat{\pi}+[\widehat{v}, \widehat{\pi}]+\frac{1}{2}[\widehat{v},[\widehat{v}, \widehat{\pi}]]\right) .
\end{aligned}
$$

La question est de savoir comment écrire $\mu_{t=1}$ en fonction de $\mu_{t=0}$. La dérivée $D U_{\widehat{\pi}_{t}}$ au point $\widehat{\pi_{t}}$ est un morphisme de complexes. En dérivant en $t$, on obtient l'équation différentielle :

$$
\frac{\partial \mu_{t}}{\partial t}=\varepsilon D U_{\widehat{\pi_{t}}}\left(\left[\widehat{v}, \widehat{\pi_{t}}\right]_{S N}\right)=\left[D U_{\widehat{\pi_{t}}}(\widehat{v}), \mu_{t}\right]_{G H} .
$$

Lemme 4. - L'opérateur $D U_{\widehat{\pi}}(\widehat{v})$ est de degré 0 (si l'on tient compte de tous les degrés impairs) et n'a pas de composantes de degré -1 dans le complexe de Hochschild.

Démonstration. - Dans notre situation linéaire, c'est le même raisonnement que dans le lemme 1 car les graphes qui interviennent ont 1 ou 2 sommets. Pour le graphe réduit à $v$, la contribution est nulle car on a une arête sortante colorée par la couleur + . Remarquons que même en degré 0 ce graphe n'intervient pas, car $v$ est nul sur $\mathfrak{h}^{\perp}{ }^{(20)}$

Remarque 4. - Notons toutefois que l'opérateur $D U_{\widehat{\pi}}(\widehat{v})$ possède a priori des composantes en tout degré positif pour le complexe de Hochschild.

Notons $Y$ le champ sur la variété de Maurer-Cartan défini au point $\nu=m+U\left(e^{\widehat{\pi}}-1\right)$ par :

$$
Y(\nu)=\left[D U_{\widehat{\pi}}(\widehat{v}), \nu\right]_{G H} .
$$

C'est plus généralement un champ de vecteurs sur la variété formelle image $U\left(T_{\text {poly }}(\mathcal{A})_{1}\right)$ (l'image par $U$ des poly-vecteurs de degré total 1 ), c'est-à-dire une co-dérivation de la cogèbre image $U\left(S^{+}\left(T_{\text {poly }}(\mathcal{A})_{1}\right)\right){ }^{(21)}$

L'équation précédente montre que la courbe intégrale du champ $Y$ relie $\mu_{t=0}$ et $\mu_{t=1}$. On en déduit que les deux structures $\mu_{t=0}$ et $\mu_{t=1}$ sont conjuguées par un élément du groupe formel des difféomorphismes de la variété formelle pointée $U\left(T_{\text {poly }}(\mathcal{A})_{1}\right)$ (le groupe à un paramètre qui intègre l'action du champ $Y$ ).

(19) On oublie l'indice $S N$ s'il n'y a pas de confusion possible.

(20) Lorsqu'on travaille sur un espace affine $f+\mathfrak{h}^{\perp}$ avec $f[\mathfrak{h}, \mathfrak{h}]=0$, ce graphe contribue comme une translation. On ne doit pas tenir compte de ce terme dans le calcul de $D U_{\widehat{\pi}}(\widehat{v})$, car le changement de supplémentaire est aussi affine.

(21) Si $V$ est un espace gradué on note $S^{+}(V)$ les éléments non constants dans $S(V[-1])$. 
Toutefois (dans le cas non gradué) d'après [19], §4.5.2, les deux structures $\mu_{t=0}$ et $\mu_{t=1}$ sont équivalentes par le groupe de jauge (des star-produits formels). En effet l'équation d'évolution s'écrit aussi

$$
\frac{\partial \mu_{t}}{\partial t}=\left[D U_{\widehat{\pi_{t}}}(\widehat{v}), Q\right]_{\mu_{t}}
$$

où $Q$ désigne le champ impair sur la variété formelle correspondant à la structure d'algèbre de Lie différentielle graduée. C’est bien l'équation de [19], §4.5.2. Par conséquent les structures sont bien conjuguées (comme série formelle en $t$ ) par le groupe de jauge. L'élément du groupe de jauge se construit de proche en proche par rapport au degré formel de la variable ${ }^{(22)} t$. Cet élément dépend évidemment du point $\mu_{t=0}$, tandis que l'élément du groupe des transformations formelles (qui est un groupe plus gros) ne dépend que du champ $\widehat{v}$ [23].

Pour avoir les idées claires, tout élément du groupe de jauge (des star-produits formels ou des $A_{\infty}$-structures) s'écrit sous la forme

$$
g=\exp \left(\varepsilon \Delta_{1}+\varepsilon^{2} \Delta_{2}+\ldots\right)
$$

où les $\Delta_{i}$ sont des éléments de degré (total) 0 dans $D_{\text {poly }}(\mathcal{A})$. Le produit se fait grâce à la formule de Campbell-Hausdorff formelle pour l'algèbre de Lie graduée de $D_{\text {poly }}(\mathcal{A})$. Pour $g=\exp (D)$, on a par définition

$$
g \cdot \mu=e^{\operatorname{ad} D} \mu .
$$

Proposition 2. - Lorsque l'on change de supplémentaire, les espaces de réduction sont isomorphes. L'isomorphisme (comme série formelle en $t$ ) est donné par l'action d'un élément du groupe de jauge de la forme

$$
\exp \left(\sum_{n \geq 1} t^{n} E_{n}\right)
$$

où les opérateurs formels (en $\varepsilon$ ) $E_{n}$ sont de degré total 0 (on tient compte de tous les degrés impairs). En particulier les structures $A_{\infty}$ données par $\mu_{t=0}$ et $\mu_{t=1}$ sont $A_{\infty}$ quasi-isomorphes (formellement en $t$ ).

Le problème délicat est que la composition pour les opérateurs polydifférentiels n'est pas associative. Par ailleurs il faut faire intervenir la notion d'éléments de type super-groupe dans les cogèbres cocommutatives colibres (voir [23] et [4]). Grâce à la bar- construction, on peut composer les morphismes de cogèbres. Si $D=\left(D_{i}\right)_{i \geq 0}$ est un élément plat (c'est-à-dire sans terme de degré -1 ) de $D_{\text {poly }}(\mathcal{A})$, on considère $\bar{D}$ la co-dérivation de cogèbre (sans co-unité) dont les coefficients de Taylor sont les $D_{i}$.

Alors $e^{\bar{D}}$ a un sens et c'est un morphisme de cogèbres. On vérifie que l'on a pour $\bar{\mu}$ (la co-dérivation de carré nul associée à $\mu$ ) :

$$
e^{\bar{D}} \bar{\mu} e^{-\bar{D}}=\overline{e^{\operatorname{ad} D} \mu}
$$

ce qui montre que, dans notre situation, les structures $A_{\infty}$ sont bien $A_{\infty}$ quasi-isomorphes.

COROLlaire 1. - Les structures $A_{\infty}$ correspondant à deux choix de supplémentaires sont $A_{\infty}$-quasi-isomorphes.

(22) Voir plus loin la construction perturbative. 
Entrelacement des espaces de cohomologie. - Fixons deux supplémentaires et notons dans cette sous-section $\mu_{1}$ et $\mu_{2}$ les structures $A_{\infty}$ correspondantes.

D'après la partie précédente il existe une série formelle en $t, D=t E_{1}+t^{2} E_{2} \ldots$ de degré total 0 tel que l'on ait $e^{\bar{D}} \bar{\mu} e^{-\bar{D}}=\overline{e^{\text {ad } D} \mu}$. On écrit $D=D_{0}+D_{1}+\cdots$ pour la décomposition dans le complexe de Hochschild décalé. L'opérateur $D_{0}$ ne prend qu'un seul argument, $D_{1}$ prend 2 arguments mais est de degré total 0 et par conséquent $D_{1}(f, g)=0$ si $f, g$ n'ont pas de composantes dans $\bigwedge \mathfrak{h}^{*}$.

On aura alors

$$
e^{\operatorname{ad} D} \mu_{1}=\mu_{2} .
$$

Si on note $\mu_{1}=\mu_{1}^{(0)}+\mu_{1}^{(1)}+\ldots$ les composantes homogènes (pour la graduation de Hochschild décalée) de $\mu_{1}$, on aura alors

$$
\left[D, \mu_{1}\right]_{G H}=\left[D_{0}, \mu_{1}^{(0)}\right]_{G H}+\ldots,
$$

par conséquent la différentielle $\mu_{1}^{(0)}$ se transforme selon le champ de la variété formelle $\xi \mapsto\left[D_{0}, \xi\right]_{G H}$. On en déduit que les deux différentielles vérifient

$$
e^{\operatorname{ad} D_{0}} \mu_{1}^{(0)}=\mu_{2}^{(0)} .
$$

Les différentielles $\mu_{1}^{(0)}$ et $\mu_{2}^{(0)}$ sont donc conjuguées par l'élément du groupe de jauge pour le complexe de Hochschild

$$
\phi: f \mapsto e^{D_{0}} f=f+D_{0}(f)+\frac{1}{2} D_{0}^{2}(f)+\cdots,
$$

c'est-à-dire que l'on a $\phi \circ \mu_{1}^{(0)}=\mu_{2}^{(0)} \circ \phi$. En particulier $\phi$ est un isomorphisme de $H^{\bullet}\left(\mathcal{A}, \mu_{1}^{(0)}\right) \operatorname{sur} H^{\bullet}\left(\mathcal{A}, \mu_{2}^{(0)}\right)$.

Calculons à l'ordre 1 dans le complexe de Hochschild (c'est-à-dire que l'on ne garde que les opérateurs au plus 2-différentiels) : on utilise la formule à l'ordre 1 en $Y$

$$
e^{X+Y}=e^{X}\left(1+\frac{1-e^{-\operatorname{ad} X}}{\operatorname{ad} X} Y\right)
$$

On calcule à l'ordre 1 :

$$
\begin{aligned}
\mu_{2}^{(0)}+\mu_{2}^{(1)} & =e^{\operatorname{ad} D_{0}+\operatorname{ad} D_{1}}\left(\mu_{1}^{(0)}+\mu_{1}^{(1)}\right)= \\
& =e^{\operatorname{ad} D_{0}}\left(1+\operatorname{ad}\left(\frac{1-e^{-\operatorname{ad} D_{0}}}{\operatorname{ad} D_{0}} D_{1}\right)\right)\left(\mu_{1}^{(0)}+\mu_{1}^{(1)}\right) \\
& =e^{\operatorname{ad} D_{0}} \mu_{1}^{(0)}+e^{\operatorname{ad} D_{0}}\left(\mu_{1}^{(1)}+\operatorname{ad}\left(\frac{1-e^{-\operatorname{ad} D_{0}}}{\operatorname{ad} D_{0}} D_{1}\right) \mu_{1}^{(0)}\right)
\end{aligned}
$$

On en déduit que l'on a

$(1.23)$

$$
\mu_{2}^{(1)}=e^{\operatorname{ad} D_{0}}\left(\mu_{1}^{(1)}+\operatorname{ad}\left(\frac{1-e^{-\operatorname{ad} D_{0}}}{\operatorname{ad} D_{0}} D_{1}\right) \mu_{1}^{(0)}\right)=e^{\operatorname{ad} D_{0}} \mu_{1}^{(1)}+\underbrace{\left[\frac{e^{\operatorname{ad} D_{0}}-1}{\operatorname{ad} D_{0}} D_{1}, \mu_{2}^{(0)}\right]}_{\text {cobord pour } \mu_{2}^{(0)}} .
$$


En conclusion, les deux produits $e^{\operatorname{ad} D_{0}} \mu_{1}^{(2)}$ et $\mu_{2}^{(2)}$ définissent les mêmes produits dans l'espace de cohomologie défini par $\mu_{2}^{(0)}$ et $\phi$ réalise un isomorphisme d'algèbres de $H^{\bullet}\left(\mathcal{A}, \mu_{1}^{(0)}\right)$ muni du produit $\mu_{1}^{(1)}$ sur $H^{\bullet}\left(\mathcal{A}, \mu_{2}^{(0)}\right)$ muni du produit $\mu_{2}^{(1)}$.

En particulier, lorsque $f, g$ sont deux fonctions dans l'espace de réduction (i.e. sans composantes dans $\left.\bigwedge \mathfrak{h}^{*}\right)$, on aura ${ }^{(23)}$ :

$$
\mu_{2}^{(1)}\left(e^{D_{0}} f, e^{D_{0}} g\right)=\left(e^{\operatorname{ad} D_{0}} \mu_{1}^{(1)}\right)\left(e^{D_{0}} f, e^{D_{0}} g\right)=e^{D_{0}}\left(\mu_{1}^{(1)}(f, g)\right),
$$

ce qui montre que l'application $\phi=e^{D_{0}}$ réalise un isomorphisme d'algèbres sur les espaces de réduction.

On résume cette section en énonçant le théorème suivant :

THÉORÈme 1. - Lorsque l'on change de supplémentaire, les espaces de réduction sont isomorphes. L'isomorphisme est donné par l'exponentielle d'un opérateur différentiel (série formelle en $t$ ) de degré 0 . C'est aussi un isomorphisme d'algèbres de l'espace de cohomologie $\left(H^{\bullet}\left(\mathcal{A}, \mu_{1}^{(0)}\right), \mu_{1}^{(1)}\right)$ sur l'espace de cohomologie $\left(H^{\bullet}\left(\mathcal{A}, \mu_{2}^{(0)}\right), \mu_{2}^{(1)}\right)$.

\subsection{Bi-quantification}

C'est une idée essentielle de l'article de Cattaneo-Felder [8].

Dans leur article, les auteurs définissent une fonction d'angle dépendant de 4 couleurs. En considérant deux sous-variétés co-isotropes de $\mathfrak{g}^{*}$, par exemple $\mathfrak{h}_{1}^{\perp}$ et $\mathfrak{h}_{2}^{\perp}$, cette fonction d'angle à 4 couleurs permet de définir sur un espace de déformation modelé $\operatorname{sur}\left(\mathfrak{h}_{1}+\mathfrak{h}_{2}\right)^{\perp}=$ $\mathfrak{h}_{1}^{\perp} \cap \mathfrak{h}_{2}^{\perp}$ une action de l'espace de réduction $H_{\varepsilon}^{\bullet}\left(\mathfrak{h}_{1}^{\perp}\right)$ à droite et une action de l'espace de réduction $H_{\varepsilon}^{\bullet}\left(\mathfrak{h}_{2}^{\perp}\right)$ à gauche.

Définition de la fonction d'angle à 4 couleurs. - Soient $\varepsilon_{1}, \varepsilon_{2}$ dans $\{-1,1\}$. Les couples $\left(\varepsilon_{1}, \varepsilon_{2}\right)$ sont les couleurs. Pour $p, q$ dans le premier quadrant ${ }^{(24)}$, on définit la fonction d'angle à 4 couleurs :

$$
\phi_{\varepsilon_{1}, \varepsilon_{2}}(p, q)=\arg (p-q)+\varepsilon_{1} \arg (p-\bar{q})+\varepsilon_{2} \arg (p+\bar{q})+\varepsilon_{1} \varepsilon_{2} \arg (p+q) .
$$

Cette fonction d'angle vérifie les propriétés de nullité résumées dans Fig. 2 où on a représenté les fonctions d'angle non nulles a priori ${ }^{(25)}$.

On va considérer les intégrales des formes d'angle associées aux graphes de Kontsevich colorés. Les variétés, sur lesquelles on intègre ces formes, sont les variétés de configurations de points dans le premier quadrant, modulo l'action des dilatations. On utilise les mêmes compactifications que celles décrites dans Kontsevich mais adaptées à notre situation.

(23) Rappelons que l'on a $\tilde{D}_{1}(f, g)=0$ lorsque le degré total de $\tilde{D}_{1}$ est nul.

(24) Les nombres complexes vérifiant $\operatorname{Im}(p)>0, \operatorname{Imp}(q)>0, \operatorname{Re}(p)>0$ et $\operatorname{Re}(q)>0$.

(25) Bien que cela soit redondant par rapport à la couleur $\left(\varepsilon_{1}, \varepsilon_{2}\right)$, dans la figure ci-dessous les arêtes en trait plein « dérivent» au bord, tandis que les arêtes en pointillé « sortent» des bords. Cette convention est compatible avec les fonctions d'angle à 2 couleurs. 

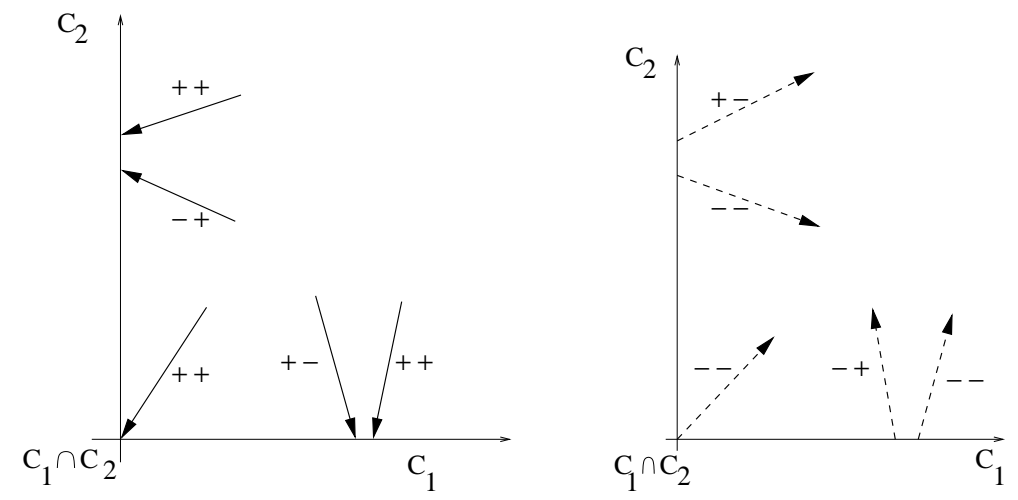

FIGURE 2. Fonction d'angle à 4 couleurs

\section{Propriétés de bord}

1. Lorsque $p, q$ se concentrent sur l'axe horizontal, les formes d'angle $\mathrm{d} \phi_{\varepsilon_{1}, \varepsilon_{2}}(p, q)$ tendent vers la 1 -forme d'angle

$$
\mathrm{d} \phi_{\varepsilon_{1}}(p, q)=\mathrm{d} \arg (p-q)+\varepsilon_{1} \mathrm{~d} \arg (p-\bar{q}) .
$$

2. Lorsque $p, q$ se concentrent sur l'axe vertical, les formes d'angle $\mathrm{d} \phi_{\varepsilon_{1}, \varepsilon_{2}}(p, q)$ tendent vers la 1-forme d'angle

$$
\mathrm{d} \phi_{\varepsilon_{2}}(p, q)=\mathrm{d} \arg (p-q)+\varepsilon_{2} \mathrm{~d} \arg (p+\bar{q}) .
$$

En conséquence, dans la formule de Stokes, les variétés qui apparaissent dans les concentrations près de l'axe horizontal et de l'axe vertical correspondent aux variétés de configurations dans le demi-plan de Poincaré avec formes d'angle à 2 couleurs décrites dans le $\$ 1.3$. On retrouve la situation avec une seule variété co-isotrope $C_{1}$ ou $C_{2}$.

Graphes à 4 couleurs. - On considère un graphe $\Gamma$ numéroté et coloré par nos 4 couleurs. Ce graphe est dessiné dans le premier quadrant. On place les points de première espèce à l'intérieur du quadrant et les points de seconde espèce sur les axes ou à l'origine. Les arêtes issues des points de seconde espèce (ainsi que les arêtes arrivant sur les points de seconde espèce) sont colorées comme dans la figure 2. Certaines arêtes, de couleur $(-,-)$, peuvent ne pas avoir de but, on dira qu'elles partent à l'infini.

Ces données permettent de définir un coefficient $w_{\Gamma}$ comme dans le $\S 1.3$.

Définition de la structure de bi-module. - Soient $C_{1}$ (par exemple $\mathfrak{h}_{1}^{\perp}$ ) et $C_{2}$ (par exemple $\mathfrak{h}_{2}^{\perp}$ ) deux sous-variétés co-isotropes. On fixe un système de coordonnées et on suppose que l'on a localement une situation d'intersection normale. On peut donc identifier le fibré normal de $C_{i}$ à un voisinage de $C_{i}(i=1,2)$.

On considère un graphe de Kontsevich à 4 couleurs. Aux sommets de première espèce on place un bivecteur de Poisson ${ }^{(26)}$.

(26) Ici $\pi$ est un bi-vecteur de Poisson général. Dans le cas des algèbres de Lie, on considérera la moitié du crochet de Lie.

$4^{\mathrm{e}}$ SÉRIE - TOME $41-2008-\mathrm{N}^{\mathrm{o}} 5$ 
Aux sommets de l'axe horizontal, on place des poly-vecteurs restreints à $C_{1}$ avec dérivées normales par rapport à $C_{1}$ (couleurs $(-,+)$ ou $(-,-)$ ). Dans notre cas il s'agit d'éléments $\operatorname{de} \mathcal{A}_{1}:=\mathcal{C}_{\text {poly }}\left(\mathfrak{h}_{1}^{\perp}\right)[[\varepsilon]] \otimes \bigwedge \mathfrak{h}_{1}^{*}$.

Aux sommets de l'axe vertical, on place des poly-vecteurs restreints à $C_{2}$ avec dérivées normales par rapport à $C_{2}$ (couleurs $(+,-)$ ou $(-,-)$ ). Dans notre cas il s'agit d'éléments $\operatorname{de} \mathcal{A}_{2}:=\mathcal{C}_{\text {poly }}\left(\mathfrak{h}_{2}^{\perp}\right)[[\varepsilon]] \otimes \bigwedge \mathfrak{h}_{2}^{*}$.

À l'origine, on place un poly-vecteur restreint à $C_{1} \cap C_{2}$ avec dérivées normales à $C_{1}$ et $C_{2}$ (couleur - - ). Dans notre cas il s'agit d'un élément de $\mathcal{A}_{1,2}:=\mathcal{C}_{\text {poly }}\left(\mathfrak{h}_{1}^{\perp} \cap \mathfrak{h}_{2}^{\perp}\right)[[\varepsilon]] \otimes \bigwedge\left(\mathfrak{h}_{1}^{*} \cap \mathfrak{h}_{2}^{*}\right)$.

Comme au $\S 1.3$ on utilise la règle complémentaire de dérivation suivante : si la variable est

1. dans $C_{1}$ et dans $C_{2}$, la couleur sera $\left(\varepsilon_{1}, \varepsilon_{2}\right)=(+,+)$

2. dans $C_{1}$ mais pas dans $C_{2}$, la couleur sera $\left(\varepsilon_{1}, \varepsilon_{2}\right)=(+,-)$

3. dans $C_{2}$ mais pas dans $C_{1}$, la couleur sera $\left(\varepsilon_{1}, \varepsilon_{2}\right)=(-,+)$

4. ni dans $C_{1}$ ni dans $C_{2}$, la couleur sera $\left(\varepsilon_{1}, \varepsilon_{2}\right)=(-,-)$.

Après restriction à $C_{1} \cap C_{2}$, on définit un élément de $\mathcal{A}_{1,2}$ et un opérateur

$$
B_{\Gamma}: \mathcal{A}_{2} \otimes \mathcal{A}_{1,2} \otimes \mathcal{A}_{1} \longrightarrow \mathcal{A}_{1,2}
$$

On considère alors $\sum_{\Gamma} w_{\Gamma} B_{\Gamma}$, où la somme porte sur tous les graphes colorés à 4 couleurs. C'est une opération tri-linéaire de $\mathcal{A}_{2} \otimes \mathcal{A}_{1,2} \otimes \mathcal{A}_{1}$ dans $\mathcal{A}_{1,2}$.

On note $\underset{1}{\star}$ le produit de droite et $\underset{2}{\star}$ le produit de gauche. 
Définition de l'espace de réduction de deuxième espèce de l'intersection $C_{1} \cap C_{2}$

Hypothèse 1. - On fait l'hypothèse de courbure nulle, c'est-à-dire $F_{\pi, C_{i}}=0$ pour $i=1$ et $i=2$ (procédure de quantification du $\S 1.4$ ).

On considère l'opérateur associé aux contributions de tous les graphes avec un sommet terrestre placé à l'origine. C'est un opérateur qui agit sur les poly-vecteurs $\operatorname{sur} C_{1} \cap C_{2}$ (avec dérivées transverses à $C_{1} \cup C_{2}$ ). Cet opérateur est encore, grâce à la formule de Stokes et l'hypothèse 1 ci-dessus, une différentielle notée $\mathcal{A}_{\pi, C_{1}, C_{2}}$. L'espace de réduction pour $C_{1} \cap C_{2}$, correspond à la cohomologie associée à cet opérateur.

Hypothèse 2. - On suppose que la fonction constante égale à 1 est dans l'espace de cohomologie, c'est-à-dire $\mathcal{A}_{\pi, C_{1}, C_{2}}(1)=0 .{ }^{(27)}$

Remarque 5. - Ces hypothèses sont automatiquement vérifiées dans le cas des bi-vecteurs de Poisson linéaires.

Compatibilité en cohomologie. - Expliquons, par exemple, la compatibilité à droite de l'action $\star$ en cohomologie : il suffit d'examiner les strates terrestres qui vont intervenir dans les différents bords.

Soit $f$ dans l'espace de cohomologie pour la différentielle $\mathcal{A}_{\pi, C_{1}, C_{2}}$ et soient $g$ et $h$ dans l'espace de cohomologie pour la différentielle $\mathcal{A}_{\pi, C_{1}}$.

On place $f$ à l'origine, $g$ et $h$ sur l'axe horizontal.

La formule de Stokes, appliquée dans ce contexte, va donner une compatibilité en cohomologie.

- Les concentrations sur les axes sont nulles (Fig. 3 CAS G, Fig. 3 CAS H) grâce à l'hypothèse 1 de courbure nulle ci-dessus.

- Il est presque clair qu'avec ces conventions, les concentrations horizontales (Fig. 3 CAS A, Fig. 3 CAS B) redonnent la différentielle $\mathcal{A}_{\pi, C_{1}}$ d'une seule variété co-isotrope agissant sur $g$ ou $h$.

- Les concentrations (Fig. 3 CAS C) donnent la différentielle $\mathcal{A}_{\pi, C_{1}, C_{2}}$ de la variété $C_{1} \cap C_{2}$ agissant $\operatorname{sur} f$.

- La concentration (Fig. 3 CAS E) donne un terme de la forme

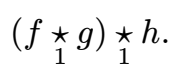

- Les concentrations (Fig. 3 CAS F) donnent un terme de la forme

$$
f \underset{1}{\star}(g \underset{\mathrm{CF}}{\star} h) .
$$

- Il reste les concentrations (Fig. 3 CAS D) qui produisent un cobord pour $C_{1} \cap C_{2}$.

(27) Cette hypothèse est analogue à l'hypothèse sur la courbure pour les sous-variétés $C_{i}$. En d'autres termes on suppose que la contribution des graphes, avec aucun point sur les axes ni à l'origine, est nulle. Elle assure que notre espace de réduction n'est pas nul.

$4^{\mathrm{e}}$ SÉRIE - TOME $41-2008-\mathrm{N}^{\mathrm{o}} 5$ 


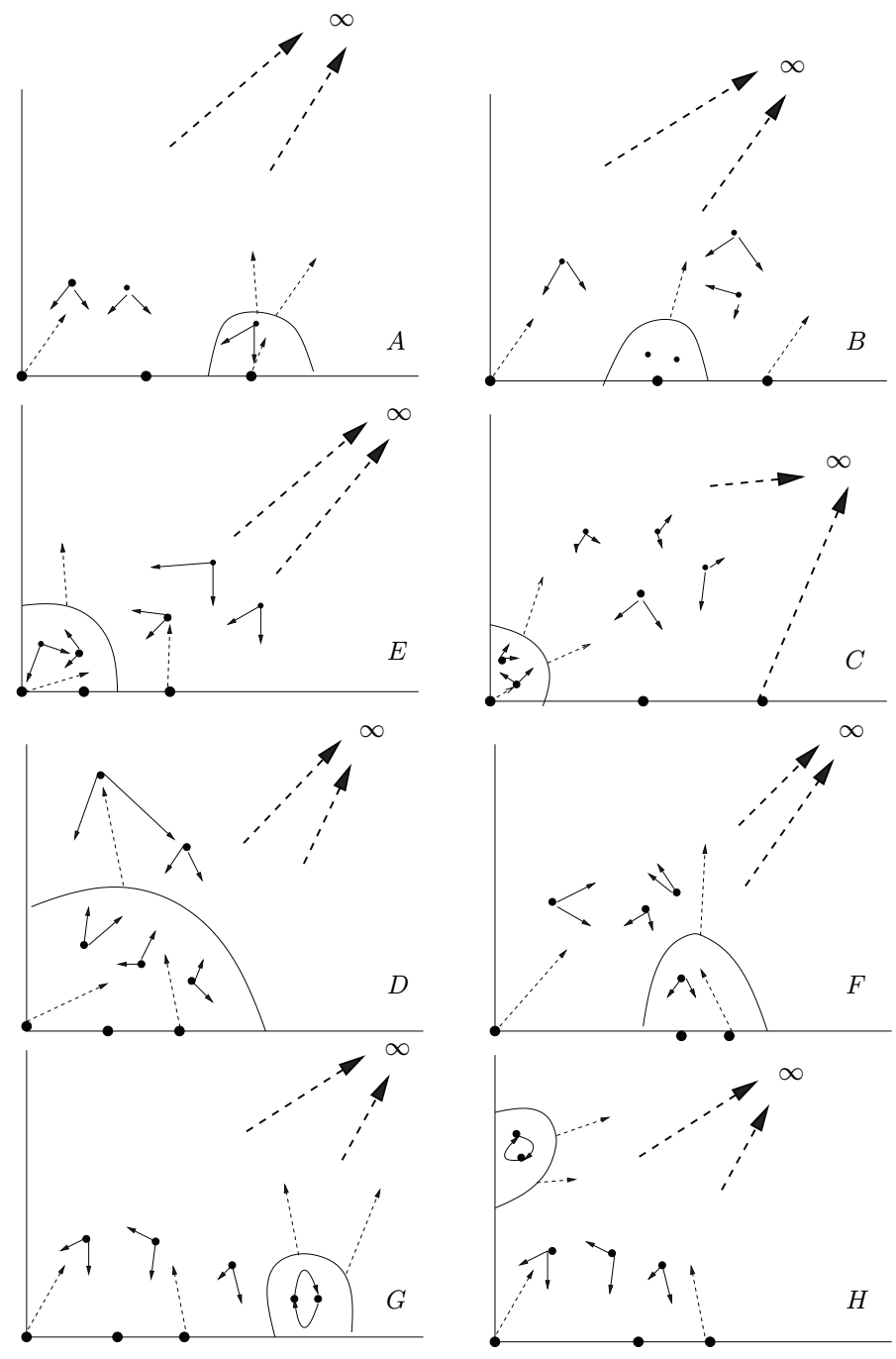

FIGURE 3. Diverses strates pour la compatibilité de l'action à droite en cohomologie

La compatibilité des actions à gauche et à droite résulte de la formule de Stokes comme toujours. Les strates de bord de type Fig. 3 CAS D fournissent un cobord de l'espace de réduction de $C_{1} \cap C_{2}$, donc la compatibilité des actions à droite n'est vraie qu'en cohomologie.

En considérant deux points terrestres (un à l'origine et l'autre sur l'axe des abscisses) on en déduirait le lemme suivant.

Lemme 5. - L'action des cobords à droite (ou à gauche) sur les cocycles fournit un cobord dans l'espace de $C_{1} \cap C_{2}$.

On peut donc conclure cette section par la compatibilité en cohomologie. 
Proposition 3. - La description en termes de diagrammes de Feynman fournit une action en cohomologie des espaces de réduction de $C_{1}$ à droite et de $C_{2}$ à gauche sur l'espace de cohomologie de réduction de deuxième espèce associé à $C_{1} \cap C_{2}$.

En degré 0 on retrouve le résultat de Cattaneo-Felder [7].

Corollaire 2. - Au niveau des fonctions, on a une action à droite et à gauche des espaces de réduction de $C_{1}$ et de $C_{2}$ sur l'espace de réduction de deuxième espèce de $C_{1} \cap C_{2}$.

Remarque 6. - On fera attention au fait que la définition de l'espace de réduction de l'intersection, appelé de deuxième espèce, est différent de l'espace de réduction de première espèce (pour les variétés co-isotropes). D'ailleurs $C_{1} \cap C_{2}$ n'est pas une sous-variété co-isotrope, on ne peut donc pas appliquer la procédure de quantification $\mathrm{du} \S 1.4$.

\section{Exemples d'espaces de réduction}

Prenons $\Gamma$ un graphe coloré. Énonçons un lemme utile dans la simplification des calculs.

Lemme 6. - Si $\Gamma$ a un sommet de la forme $\stackrel{x}{\bullet} \leftarrow \boldsymbol{\bullet}_{\bullet--\rightarrow}^{\boldsymbol{y}}$, alors $w_{\Gamma}=0$.

Démonstration. - Pour un tel graphe, la forme $\Omega_{\Gamma}$ (que l'on intègre) présente à ce sommet la configuration suivante :

$$
\stackrel{x}{\bullet} \stackrel{z}{\bullet}-\rightarrow^{y}=\stackrel{x}{=} \leftarrow^{z} \leftarrow \leftarrow^{y} .
$$

Les dérivées en $z$ dans $\Omega_{\Gamma}$ ne proviennent que de cette partie du diagramme, donc le calcul se fait à $x, y$ fixés. On a donc $w_{\Gamma}=0$ d'après le lemme du $§ 7.3 .3 .1$ de [19].

\subsection{Cas des paires symétriques (ou cas d'un supplémentaire stable)}

On se place dans le cas des paires symétriques. On considère un caractère $\lambda$ de $\mathfrak{k}$, c'est-àdire une forme linéaire sur $\mathfrak{k}$ telle que $\lambda([\mathfrak{k}, \mathfrak{k}])=0$. Alors $\lambda+\mathfrak{k}^{\perp}$ est l'espace affine des formes linéaires dont la restriction à $\mathfrak{k}$ vaut $\lambda$. C'est une sous-variété co-isotrope.

Proposition 4. - Dans le cas des paires symétriques (et plus généralement dans le cas où $\mathfrak{h}$ admet un supplémentaire stable sous l'action adjointe de $\mathfrak{h}$ ), on a $A_{\pi}=\varepsilon d_{C E}$. Par conséquent l'espace de réduction pour la sous-variété $\lambda+\mathfrak{k}^{\perp}$ est

$$
H_{\varepsilon}^{0}\left(\lambda+\mathfrak{k}^{\perp}\right)=\mathcal{C}_{\text {poly }}\left(\lambda+\mathfrak{k}^{\perp}\right)^{\mathfrak{k}}[[\varepsilon]]=S(\mathfrak{p})^{\mathfrak{k}}[[\varepsilon]] .
$$

Démonstration. - La démonstration se base sur l'inventaire des graphes qui peuvent apparaître dans $A_{\pi}$. Pour une paire symétrique (et plus généralement si $\mathfrak{h}$ admet un supplémentaire stable), les crochets vérifient $[\mathfrak{k}, \mathfrak{p}] \subset \mathfrak{p}$, ce qui impose certaines restrictions sur les couleurs des graphes. On place au point terrestre un élément $\phi \in \mathcal{C}_{\text {poly }}\left(\lambda+\mathfrak{k}^{\perp}\right)[[\varepsilon]] \otimes \bigwedge^{\mathfrak{k}^{*}}$ de degré $q$. Il sort nécessairement $q+1$ arêtes à l'infini, donc au moins une arête sort d'un point aérien du graphe $\Gamma$. Pour $n \geq 2$ les graphes qui interviennent sont de deux types.

(i) Si dans le graphe aérien on a une arête colorée park* qui sort à l'infini, alors au sommet de sortie on a dans le graphe $\Gamma$ :

- soit deux arêtes de même couleur qui se suivent (car on a $[\mathfrak{k}, \mathfrak{p}] \subset \mathfrak{p}$ et $[\mathfrak{k}, \mathfrak{k}] \subset \mathfrak{k}$ ) et le coefficient $w_{\Gamma}$ vaut 0 d'après le lemme 6 ,

$4^{\text {e }}$ SÉRIE - TOME $41-2008-\mathrm{N}^{\mathrm{o}} 5$ 
- soit une seule arête (colorée nécessairement par $\mathfrak{p}^{*} \operatorname{car} \lambda[\mathfrak{k}, \mathfrak{k}]=0$ ), auquel cas le coefficient $w_{\Gamma}$ est encore nul car $n \geq 2$ et ce sommet ne contribue qu'une fois ${ }^{(28)}$ dans la forme $\Omega_{\Gamma}$.

(ii) Si deux arêtes colorées par $\mathfrak{k}^{*}$ sortent d'un même sommet, alors ce sommet ne contribue qu'au plus une fois dans la forme différentielle. Comme $n \geq 2$, le coefficient sera nul.

On conclut que les arêtes qui sortent à l'infini proviennent de l'axe réel. Comme au moins une arête sort d'un sommet aérien, toutes les contributions pour $n \geq 2$ sont nulles.

En conséquence seuls les graphes pour $n=1$ interviennent et on trouve $\mathrm{d}_{C E}(\phi)=0$, où $d_{C E}$ est la différentielle de Cartan-Eilenberg.

En spécialisant en $\varepsilon=1$ pour les polynômes en $\varepsilon$, on obtient le corollaire suivant :

Corollaire 3. - Les constructions de Cattaneo-Felder munissent $H^{\bullet}(\mathfrak{k}, S(\mathfrak{p}))$ d'une structure d'algèbre associative.

\subsection{Cas linéaire pour les fonctions}

Plaçons-nous dans le cas linéaire et prenons $\mathfrak{h}^{\perp}$ comme variété co-isotrope. On ne suppose pas que $\mathfrak{h}$ admet un supplémentaire stable.

Graphes intervenant dans le calcul de $A_{\pi}$ pour les fonctions. - Lorsqu'on place au sommet terrestre une fonction, les graphes qui interviennent dans le calcul de la différentielle $A_{\pi}$ sont de trois types ${ }^{(29)}$ :

$(2.1)$

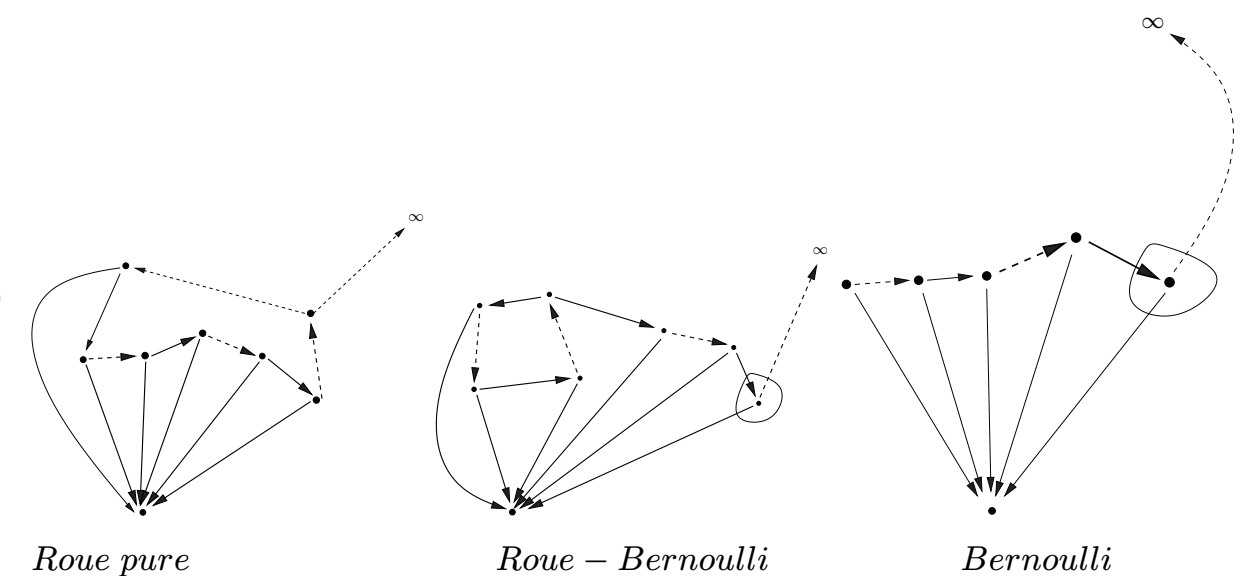

1. les graphes de type Bernoulli, avec la dernière arête partant à l'infini ( $c f$. (2.1) dessin de droite);

2. les graphes de type Roue, avec des rayons attachés directement à l'axe réel sauf pour l'un d'entre eux qui est attaché à un graphe de type Bernoulli dont la dernière arête part à l'infini (cf. (2.1) dessin du milieu);

3. les graphes de type Roue avec des rayons attachés directement à l'axe réel sauf pour l'un d'entre eux qui part à l'infini (cf. (2.1) dessin de gauche).

(28) Ce sommet se déplace sur 2 dimensions, tandis que cette variable ne contribue qu'une fois dans $\Omega_{\Gamma}$.

(29) On pourrait regrouper les deux derniers types. 
En effet ce sont les mêmes graphes que ceux que l'on rencontre dans la formule de Kontsevich $f_{\text {Kont }}^{\star} g$ pour les algèbres de Lie, lorsqu'on prend pour $g$ une forme linéaire. La forme linéaire correspond ici à la direction qui part à l'infini.

\section{Parité et homogénéité}

Lemme 7. - Les coefficients associés aux graphes intervenant dans le calcul de $A_{\pi}(f)$ sont nuls si le nombre de sommets aériens est pair.

Démonstration. - La variété de configurations admet une symétrie par rapport à l'axe vertical. S'il y a $n$ sommets aériens, il y a $2 n-1$ arêtes (car l'une d'entre elles part à l'infini). En faisant agir la symétrie, on trouve un facteur $(-1)^{n+1}$, ce qui montre que $n$ doit être impair pour que le coefficient soit non nul.

Lemme 8. - Si $f=f_{0}+\varepsilon f_{1}+\varepsilon^{2} f_{2}+\ldots$ est dans l'espace de réduction, alors $F=f_{0}+$ $\varepsilon^{2} f_{2}+\varepsilon^{4} f_{4}+\ldots$ l'est aussi et l'on peut prendre $F$ homogène si l'on tient compte du degré de $\varepsilon$ (qui vaut par convention 1 ).

Démonstration. - Les graphes qui interviennent dans les équations de réduction sont de trois types :

1. Bernoulli $B_{n}$ : l'opérateur dérive $n$ fois mais ajoute un degré (à cause de la racine), le degré total (y compris $\varepsilon$ ) est donc 1 .

2. Roue (attachée ou non à un Bernoulli ) : l'opérateur dérive $n-1$ fois, le degré total (y compris $\varepsilon$ ) est donc 1 .

Les graphes avec un nombre pair de sommets n'interviennent pas, donc les termes modulo 2 vérifient encore le système de réduction.

Les équations s'écrivent (où les $D_{n}$ désignent les contributions des graphes avec $n$ sommets) :

$$
D_{1}\left(f_{0}\right)=0, \quad D_{1}\left(f_{2}\right)+D_{3}\left(f_{0}\right)=0 \quad \ldots
$$

donc les composantes homogènes (en tenant compte de $\varepsilon$ ) vérifient encore le système.

\subsection{Cas linéaire pour les poly-vecteurs}

Décrivons les graphes qui vont intervenir dans le calcul de la différentielle $A_{\pi}$ lorsqu'on l'applique sur un élément de $\mathcal{A}=\mathcal{C}^{\infty}\left(\mathfrak{h}^{\perp}\right)[[\varepsilon]] \otimes \wedge \mathfrak{h}^{*}$, où $\mathfrak{h}$ est encore une sous-algèbre quelconque de $\mathfrak{g}$.

Lemme 9. - Les graphes intervenant dans le calcul de la différentielle des éléments de $\mathcal{A}$ sont de deux types: les graphes déjà rencontrés pour les fonctions (la composante dans $\bigwedge \mathfrak{h}^{*}$ n'intervient pas) et les graphes qui sont le crochet de deux Bernoulli, avec une dérivation unique de la racine par une arête issue de l'axe réel (voir (2.2) dessin de droite).

Démonstration. - Deux cas se présentent.

1. Toutes les arêtes issues de l'axe réel vont à l'infini; dans ce cas les graphes qui interviennent sont ceux que l'on a détaillés pour les fonctions. 
2. Une ou plusieurs arêtes, issues de l'axe réel, vont sur un sommet aérien. Ces sommets sont des racines de graphes de type Lie (dont certaines arêtes pourraient partir à l'infini).

Il n'y a qu'une arête issue de l'axe réel qui va vers un sommet aérien, sinon la forme à intégrer serait à variables séparées et on aurait un problème de dimension (à cause du groupe de dilatation).

De ce graphe il part 2 arêtes à l'infini. Raisonnons sur le symbole $\Gamma(X, Y)$ de ce graphe. Le mot $\Gamma(X, Y)$ doit être de type Lie et de degré deux en $Y$ (représentant les deux arêtes qui partent à l'infini). Le mot s'écrit sous la forme $[A, B]$. Comme l'arête issue de l'axe réel dérive le sommet du graphe, les mots $A$ et $B$ doivent contenir $Y$, sinon $A=X$ ou $B=X$ et on aurait une arête double comme dans (2.2) (dessin de gauche ${ }^{(30)}$ ). En conséquence les mots $A$ et $B$ sont des mots de type Bernoulli c'est-à-dire de la forme

$$
[\underbrace{X,[X, \ldots,[X}_{n \text { fois }}, Y]]]
$$

l'arête partant à l'infini étant placée en dernière position. Ces graphes sont comme dans (2.2) (dessin de droite). Lorsque les mots sont réduits à $Y$ on retrouve le graphe intervenant dans la différentielle de Hochschild.

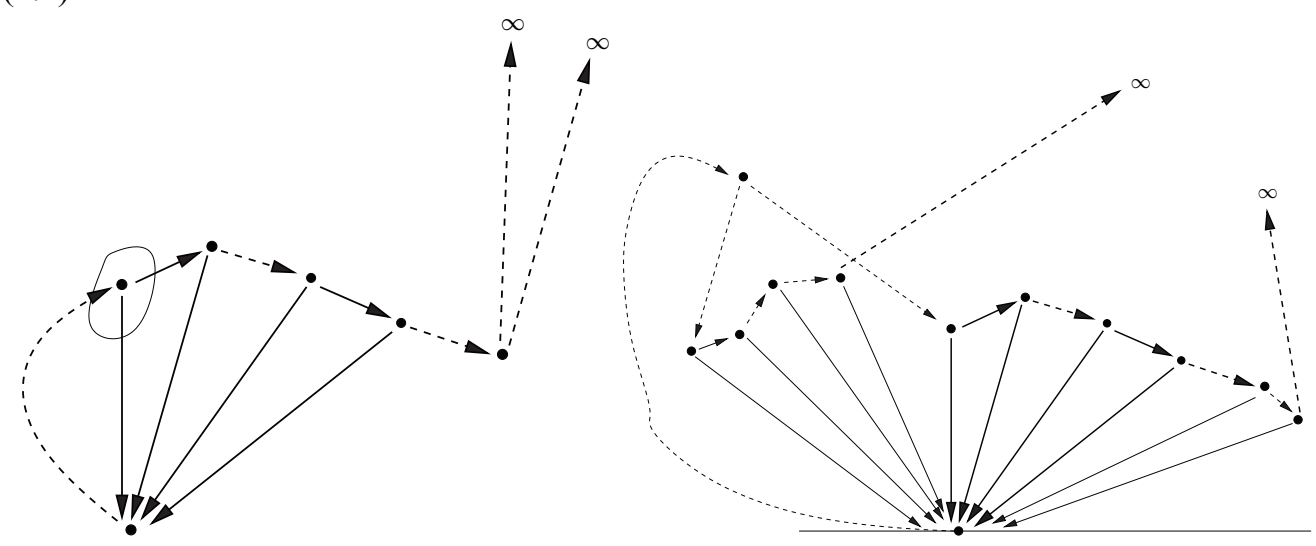

\subsection{Cas Iwasawa}

Considérons une paire symétrique. Nous avons défini dans [32], une notion de décomposition d'Iwasawa, généralisant la décomposition d'Iwasawa pour les paires symétriques réductives. Pour simplifier la lecture de cet article, rappelons brièvement cette construction qui intervient dans la définition de l'homomorphisme d'Harish-Chandra généralisé.

(30) N'oublions pas que la couleur inverse le sens de l'arête. 
Rappels sur la notion de décomposition d'Iwasawa généralisée. - Pour $\xi \in \mathfrak{k}^{\perp}$ on note $\mathfrak{g}(\xi)$ le centralisateur de $\xi$ pour l'action co-adjointe. C'est une sous-paire symétrique algébrique ; elle admet une décomposition de Cartan notée $\mathfrak{k}(\xi) \oplus \mathfrak{p}(\xi)$.

Lorsque la dimension de $\mathfrak{g}(\xi)$ est minimale pour $\xi$ parcourant $\mathfrak{k}^{\perp}$, on dira que $\xi$ est régulier. Les éléments réguliers forment un ouvert de Zariski. On a alors $[\mathfrak{k}(\xi), \mathfrak{p}(\xi)]=0$, ce qui permet de montrer que la sous-algèbre engendrée $\operatorname{par} \mathfrak{p}(\xi)$ est nilpotente. On note $\mathfrak{s}_{\xi}$ sa partie semi-simple, c'est donc une sous-algèbre abélienne formée d'éléments semi-simples (un tore) et qui est dans $\mathfrak{p}$. On dira que $\xi$ est générique lorsque la dimension de ce tore est maximale. Ce tore généralise la notion de sous-espace de Cartan.

On peut alors décomposer $\mathfrak{g}$ sous l'action adjointe de $\mathfrak{s}_{\xi}$ lorsque ce tore est diagonalisable (déployé ${ }^{(31)}$ ). C'est ce que nous supposerons ${ }^{(32)}$. On note $\mathfrak{g}_{o}$ le centralisateur de $\mathfrak{s}_{\xi}$. C'est une sous-paire symétrique, appelée petite paire symétrique. On note $\mathfrak{g}_{o}=\mathfrak{k}_{o} \oplus \mathfrak{p}_{o}$ sa décomposition de Cartan. Comme $\mathfrak{s}_{\xi} \subset \mathfrak{p}$, si $\alpha$ est une racine alors $-\alpha$ aussi; ceci permet d'exhiber, en séparant les racines et leurs opposées, des décompositions triangulaires $: \mathfrak{g}=\mathfrak{n}_{-} \oplus \mathfrak{g}_{o} \oplus \mathfrak{n}_{+}$ et des décompositions d'Iwasawa $\mathfrak{g}=\mathfrak{k} \oplus \mathfrak{p}_{o} \oplus \mathfrak{n}_{+}$.

Espace de réduction. - On fixe une décomposition d'Iwasawa généralisée dans le cas des paires symétriques

$$
\mathfrak{g}=\mathfrak{k} \oplus \mathfrak{p}_{o} \oplus \mathfrak{n}_{+}
$$

et on note $\mathfrak{g}_{o}=\mathfrak{k}_{o} \oplus \mathfrak{p}_{o}$.

Proposition 5. - L'espace de réduction pour la sous-variété co-isotrope $\left(\mathfrak{k}_{o} \oplus \mathfrak{n}_{+}\right)^{\perp}$ est

$$
H_{\varepsilon}^{0}\left(\left(\mathfrak{k}_{o} \oplus \mathfrak{n}^{+}\right)^{\perp}\right)=\mathcal{C}_{\text {poly }}\left(\mathfrak{k}_{o}^{\perp}\right)^{\mathfrak{k}_{o}}[[\varepsilon]]=S\left(\mathfrak{p}_{o}\right)^{\mathfrak{k}_{o}}[[\varepsilon]] .
$$

Démonstration. - On fixe un supplémentaire de $\mathfrak{k}_{o}$ dans $\mathfrak{k}$ noté abusivement $\mathfrak{k} / \mathfrak{k}_{o}$. Les couleurs des arêtes sont donc:

- les arêtes colorées par $-\rightarrow$ correspondent aux variables dans $\mathfrak{n}_{+}^{*}, \mathfrak{k}_{o}^{*}$

- les arêtes colorées par $\longrightarrow$ correspondent aux variables $\mathfrak{p}_{o}^{*}$ et $\mathfrak{k}^{*} / \mathfrak{k}_{o}^{*}$.

Soit $f$ vérifiant l'équation $A_{\pi}(f)=0$. D'après le lemme 8 , on peut écrire $f=f_{0}+\varepsilon^{2} f_{2}+$ $\varepsilon^{4} f_{4}+\ldots$ avec $D_{1}\left(f_{0}\right)=0$. Donc $f_{0}$ est $\mathfrak{k}_{0} \oplus \mathfrak{n}_{+}$-invariant. D'après [32] un élément de $S\left(\mathfrak{p}_{0}\right)^{\mathfrak{k}_{0}}$.

Les graphes qui interviennent dans $D_{n}$ sont de type Bernoulli ou de type Roue attachée à un Bernoulli.

Examinons l'action de ces opérateurs sur $f_{0} \in S\left(\mathfrak{p}_{0}\right)^{\mathfrak{k}_{0}}$. Comme on ne peut dériver $f_{0}$ qu'en les directions $\mathfrak{p}_{o}^{*}$, toutes les arêtes qui arrivent sur le sommet terrestre sont colorées

(31) C'est le cas si le corps de base est $\mathbb{C}$.

(32) Cette restriction n'est pas très sévère, car il suffit de tout complexifier et de considérer la paire symétrique complexe obtenue comme une paire symétrique réelle.

$4^{\text {e }}$ SÉRIE - TOME $41-2008-\mathrm{N}^{\mathrm{o}} 5$ 
par $\longrightarrow$. L'arête sortant à l'infini est soit dans $\mathfrak{k}_{o}^{*}$, soit dans $\mathfrak{n}_{+}^{*}$.

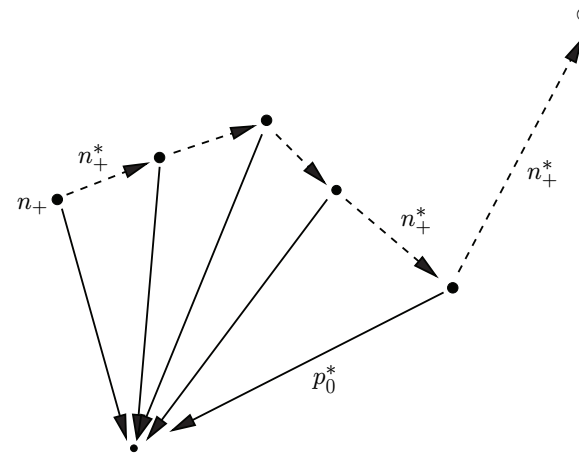

Cas 1

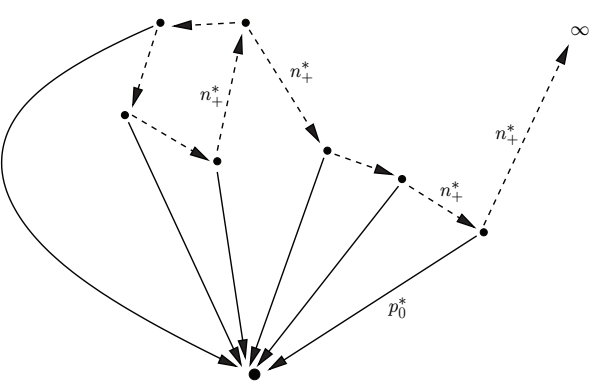

Cas 2

Premier cas. - C'est le type Bernoulli comme (2.3) (dessin de gauche).

Le coefficient sera nul, si on a deux arêtes de type $\longrightarrow$ qui se suivent au sommet où l'arête part à l'infini. Par conséquent l'arête qui part à l'infini est colorée par $\mathfrak{n}_{+}^{*}$. Par ailleurs on a $\left[\mathfrak{p}_{0}, \mathfrak{n}_{+}\right] \subset \mathfrak{n}_{+}$, donc la couleur $\rightarrow$ se propage dans le graphe. La racine du graphe représente, pour l'opérateur différentiel, un coefficient dans $\mathfrak{n}_{+}$qui est donc nul par restriction à $\left(\mathfrak{k}_{o} \oplus \mathfrak{n}_{+}\right)^{\perp}$.

Deuxième cas. - Comme (2.3) (dessin de droite), le graphe à considérer de manière générale est une roue attachée à l'axe réel et à un sous-graphe de Bernoulli (ce graphe pouvant être vide auquel cas l'arête part directement à l'infini et est colorée par $\mathfrak{n}_{+}^{*}$ ). Comme précédemment, pour que le coefficient soit non nul, l'arête qui part à l'infini est colorée par $\mathfrak{n}_{+}^{*}$. Ceci implique que l'attache sur la roue se fait dans des dérivations selon $\mathfrak{n}_{+}^{*}$ (voir (2.3) dessin de droite). La dérivation dans la roue se fait alors soit de manière homogène selon les dérivées $\mathfrak{n}_{+}^{*}$ pour la couleur $\rightarrow$, soit selon des dérivées dans la direction $\mathfrak{k}^{*} / \mathfrak{k}_{o}^{*}$ avec couleur $\rightarrow$. Dans les deux cas l'opérateur fera apparaître un terme de la forme

$$
\operatorname{tr}_{\mathfrak{n}_{+}}\left(\operatorname{ad} P_{1} \ldots \operatorname{ad} P_{l} \operatorname{ad} N_{1}\right)=0
$$

ou un terme de la forme

$$
\operatorname{tr}_{\mathfrak{g} /\left(\mathfrak{g}_{o} \oplus \mathfrak{n}_{+}\right)}\left(\operatorname{ad} P_{1} \ldots \operatorname{ad} P_{l} \operatorname{ad} N_{1}\right)=0 .
$$

L'opérateur différentiel sera alors nul quand on l'applique à des fonctions qui sont dans $S\left(\mathfrak{p}_{o}\right)^{\mathfrak{k}_{o}}$. Par conséquent le système d'équations est tout simplement $D_{1}\left(f_{p}\right)=0$.

\subsection{Cas des polarisations}

On prend $\xi \in \mathfrak{g}^{*}$ une forme linéaire. La forme bilinéaire alternée $B_{\xi}$, définie par $B_{\xi}(X, Y)=<\xi,[X, Y]>$ pour $X, Y \in \mathfrak{g}$, admet pour noyau $\mathfrak{g}(\xi)$. Une polarisation en $\xi$ dans $\mathfrak{g}$ est une sous-algèbre isotrope pour $B_{\xi}$ et de dimension maximale parmi les sous-espaces isotropes. C'est automatiquement une sous-algèbre algébrique. On dit que $\mathfrak{b}$ vérifie la condition de Pukanszky si on $\mathfrak{a} \mathfrak{b}=\mathfrak{g}(\xi)+\mathfrak{b}_{u}$, où $\mathfrak{b}_{u}$ désigne le radical unipotent de $\mathfrak{b}$.

Alors $\xi+\mathfrak{b}^{\perp}$ est une sous-variété co-isotrope. On note $B$ un sous-groupe algébrique connexe de $G$ d'algèbre de Lie $\mathfrak{b}$. 
Proposition 6. - L'espace de réduction pour la sous-variété $\xi+\mathfrak{b}^{\perp}$ est le corps $\mathbb{R}$, c'està-dire que l'on a

$$
H_{\varepsilon}^{0}\left(\xi+\mathfrak{b}^{\perp}\right)=\mathbb{R}[[\varepsilon]] .
$$

Démonstration. - Considérons une fonction $f=f_{0}+\varepsilon^{1} f_{1}+\varepsilon^{2} f_{2}+\cdots$ dans l'espace de réduction. La première équation fournit des fonctions $B$-invariantes sur $\xi+\mathfrak{b}^{\perp}$, c'est-à-dire des fonctions constantes, car $B \cdot \xi$ est ouvert dans $\xi+\mathfrak{b}^{\perp}$. Comme précédemment les autres équations se résument par récurrence aux équations $D_{1}\left(f_{n}\right)=0$ pour tout $n$.

\section{Fonction $E(X, Y)$ pour les paires symétriques}

On se place dans le cas des paires symétriques $\mathfrak{g}=\mathfrak{k} \oplus \mathfrak{p}$ et on considère le cas de la sousvariété $\lambda+\mathfrak{k}^{\perp}$ où $\lambda$ désigne un caractère de $\mathfrak{k}\left(\right.$ i.e. $\left.\lambda \in(\mathfrak{k} /[\mathfrak{k}, \mathfrak{k}])^{*}\right)$. On considère le produit $B_{\pi}$ (qui prend deux arguments) introduit au $\S 1.4$ pour $\pi$ le bi-vecteur de Poisson associé à la moitié du crochet de Lie.

\subsection{Définition de la fonction $E(X, Y)$ pour les paires symétriques}

Pour $X, Y \in \mathfrak{p}$ on considère les fonctions exponentielles $e^{X}$ et $e^{Y}$. On considère alors $B_{\pi}\left(e^{X}, e^{Y}\right)$ que l'on restreint à $\lambda+\mathfrak{k}^{\perp}$. On pourra consulter [3] pour une description précise des graphes dans le cas linéaire.

Lemme 10. - Dans le calcul de $B_{\pi}\left(e^{X}, e^{Y}\right)$ les graphes de type Lie avec racine dans $\mathfrak{p}$ n'interviennent pas.

Démonstration. - D'après le lemme 6, si la racine d'un tel graphe est dans $\mathfrak{p}$, alors le poids associé est nul car le graphe présente, à la racine, un sommet de la forme

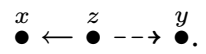

Dans le cas des paires symétriques, seuls les graphes avec racine dans $\mathfrak{k}$ ou de type Roue interviennent, donc par restriction on trouve l'action du caractère. Comme d'habitude, les graphes sont des superpositions de graphes simples de type Lie ou Roue. Le résultat est donc une exponentielle.

La composante logarithmique de $B_{\pi}\left(e^{X}, e^{Y}\right)$ dans p est donc $X+Y$ et celle dans ḱ s'écrit $H(X, Y)$, avant restriction à $\lambda+\mathfrak{k}^{\perp}$.

Lorsque $\lambda=0$, seuls les graphes de type Roue vont intervenir de manière significative.

DÉfinition 4. - On note $E(X, Y)$ les contributions de tous les graphes de type Roue. C'est une fonction analytique en $X, Y$ près de 0 .

On a donc :

$$
B_{\pi}\left(e^{X}, e^{Y}\right)=E_{\lambda}(X, Y) e^{X+Y},
$$

où on a noté $E_{\lambda}(X, Y):=e^{\lambda(H(X, Y))} E(X, Y)$.

La fonction $E_{\lambda}(X, Y)$ est le symbole formel du star-produit de Cattaneo-Felder associé à la sous-variété co-isotrope $\lambda+\mathfrak{k}^{\perp}$.

Lemme 11. - On a les symétries suivantes $E_{\lambda}(X, Y)=E_{-\lambda}(Y, X)$ et $E_{\lambda}(-X,-Y)=$ $E_{\lambda}(X, Y)$

$4^{\text {e }}$ SÉRIE - TOME $41-2008-\mathrm{N}^{\mathrm{o}} 5$ 
Démonstration. - Dans le cycle d'une roue, lorsqu'une sortie est colorée par $\mathfrak{p}^{*}$ il se produit un changement de couleur dans le cycle. En définitive, le nombre de changements de couleur est pair, c'est-à-dire qu'un tel cycle est attaché à un nombre pair de sorties dans $\mathfrak{p}$, disons $2 p$ et à un nombre $q$ de sorties dans $\mathfrak{k}$. Par ailleurs un sous-graphe (de type Lie) dont la racine est dans $\mathfrak{p}$ a un nombre pair de sommets, et chaque graphe dont la racine est dans $\mathfrak{k}$ a un nombre impair de sommets (car les "pattes" de ces graphes sont attachées sur $e^{X}, e^{Y}$, c'est-à-dire que les pattes sont colorées par $\left.\mathfrak{p}^{*}\right)$. Il y a donc $2 p+q$ sommets dans le cycle et $q(\bmod 2)$ sommets en dehors du cycle. Au total on a un nombre pair de sommets dans $\Gamma$ et donc $E(-X,-Y)=E(X, Y)$.

Faisons une symétrie axiale. On a

$$
\Gamma^{\vee}(X, Y)=\Gamma(Y, X) \quad \text { et } \quad w_{\Gamma^{\vee}}=(-1)^{\sharp\left\{\mathrm{E}_{\Gamma}\right\}} w_{\Gamma}=w_{\Gamma},
$$

ce qui montre la symétrie recherchée.

Pour les graphes de type Lie, qui contribuent pour la composante dans $\mathfrak{k}$, on obtient $w_{\Gamma^{\vee}}=$ $-w_{\Gamma}$, car on a un nombre impair de sommets aériens. Les mots de Lie qui sont dans $\mathfrak{k}$ sont nécessairement pairs.

Définition 5. - On définit le produit de Cattaneo-Felder $\underset{C F, \lambda}{\star}$ sur $S(\mathfrak{p})$ par la relation pour $f, g \in S(\mathfrak{p})=\mathcal{C}_{\text {poly }}\left(\mathfrak{k}^{\perp}\right)$ et $\xi \in \mathfrak{k}^{\perp}=\mathfrak{p}^{*}$ :

$$
\left(f_{\mathrm{CF}, \lambda}^{\star} g\right)(\xi)=B_{\pi}(f, g)=\left.E_{\lambda}\left(\partial_{\nu}, \partial_{\eta}\right)(f \otimes g)\right|_{\nu=\eta=\xi} .
$$

Pour $\lambda=0$, on notera ce produit $\underset{\mathrm{CF}}{\star}$. D'après la proposition 4 et $(3.2)$ on déduit le lemme suivant.

Lemme 12. - Le produit $\underset{\mathrm{CF}, \lambda}{\star}$ est associatif sur $S(\mathfrak{p})^{\mathfrak{k}}$ et vérifie $f_{C F, \lambda}^{\star} g=g_{\mathrm{CF},-\lambda}^{\star} f$.

Le corollaire suivant précise le corollaire 3 (§2.1) et décrit ce qui se passe en cohomologie.

Corollaire 4. - Le produit associatif de Cattaneo-Felder dans $H^{\bullet}(\mathfrak{k}, S(\mathfrak{p}))$ est donné par l'action de l'opérateur E sur les coefficients. Le produit en cohomologie est donc l'extension du produit sur $S(\mathfrak{p})^{\mathfrak{k}}$.

Démonstration. - Si une arête issue de l'axe réel intervient dans un graphe, il existerait une arête colorée par $\mathfrak{k}^{*}$ qui sortirait à l'infini. Or au sommet d'où part une telle arête, on aurait deux arêtes de même couleur qui se suivent et donc le coefficient serait nul par le lemme 6 . 


\subsection{Contributions dans $E(X, Y)$}

Dans cette section, nous exploitons une symétrie pour les graphes qui interviennent dans $E(X, Y)$. Pour $X, Y \in \mathfrak{p}$ on note $x=\operatorname{ad} X$ et $y=\operatorname{ad} Y$ les opérateurs adjoints.

Proposition 7. - Dans le logarithme de $E(X, Y)$, interviennent des termes de la forme

$$
w_{\Gamma}\left(\operatorname{tr}_{\mathfrak{p}}\left(x_{1} \cdots x_{n}\right)+(-1)^{n-1} \operatorname{tr}_{\mathfrak{k}}\left(x_{n} \cdots x_{2} x_{1}\right)\right)
$$

où $w_{\Gamma}$ est le coefficient associé à un graphe coloré de type Roue, $x_{i}=\operatorname{ad} X_{i}$ et $X_{i}$ est un mot de type Lie en $X, Y$.

Démonstration. - On change l'orientation et la coloration des arêtes dans le cycle pour conserver, au signe près, la forme $\Omega_{\Gamma}$.

Par exemple dans Fig. 4, les arêtes 2, 4, 6, 8, 10 se retrouvent dans la forme $\Omega_{\Gamma}$, en position $10,8,6,4,2$. Comme on a inversé le sens des arêtes, la forme est la même au signe près, donné par la signature de l'inversion de numérotation dans le cycle; sur l'exemple Fig. 4 le signe est donc $(-1)^{4}=1$. On trouve, dans $\ln (E(X, Y))$, une contribution de la forme

$$
w_{\Gamma}\left(\operatorname{tr}_{\mathfrak{p}}\left(x_{5} x_{1} x_{2} x_{3} x_{4}\right)+\operatorname{tr}_{\mathfrak{p}}\left(x_{5} x_{4} x_{3} x_{2} x_{1}\right)\right) .
$$
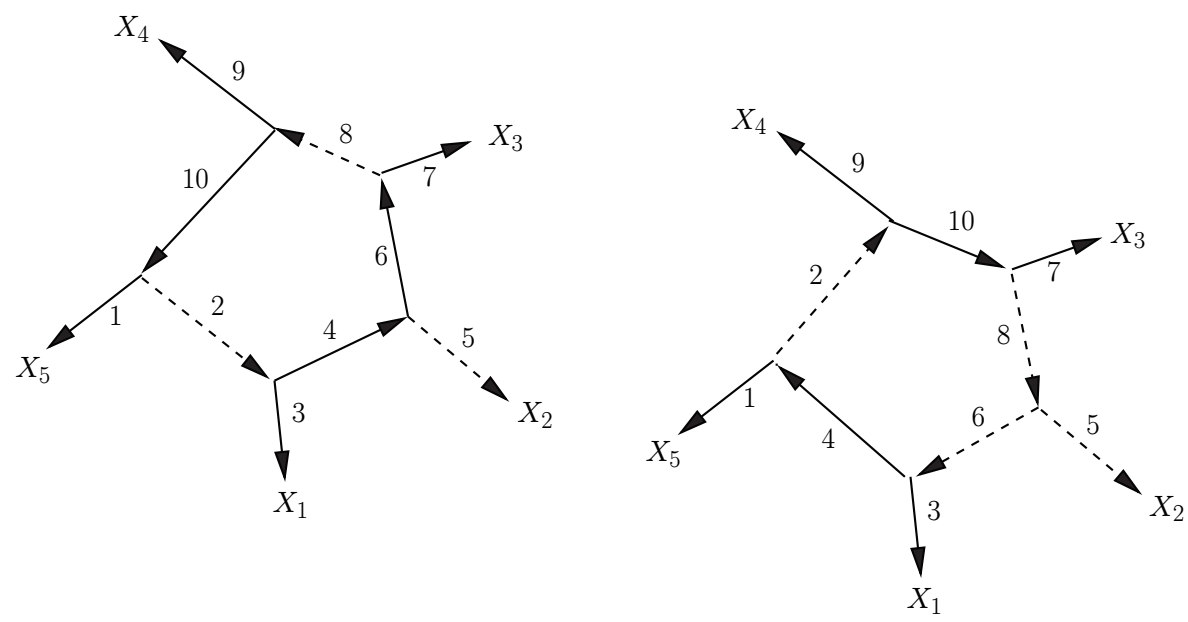

Figure 4. Roue dans un sens et dans l'autre

En général si l'un des $X_{i}$ est dans $\mathfrak{p}$, par exemple $X_{1}$ recevant la couleur $\mathfrak{p}^{*}$ dans la roue, alors on trouve des contributions de la forme :

$$
\operatorname{tr}_{\mathfrak{p}}\left(x_{1} \cdots x_{n}\right)
$$

et

$$
(-1)^{n-1} \operatorname{tr}_{\mathfrak{p}}\left(x_{1} \cdot x_{n} \cdot x_{n-1} \cdots x_{2}\right)=(-1)^{n-1} \operatorname{tr}_{\mathfrak{k}}\left(x_{n} \cdots x_{2} x_{1}\right) .
$$

$\mathrm{Au}$ total on a une contribution de la forme :

$$
\operatorname{tr}_{\mathfrak{p}}\left(x_{1} \cdots x_{n}\right)+(-1)^{n-1} \operatorname{tr}_{\mathfrak{p}}\left(x_{1} x_{n} \cdots x_{2}\right)=\operatorname{tr}_{\mathfrak{p}}\left(x_{1} \cdots x_{n}\right)+(-1)^{n-1} \operatorname{tr}_{\mathfrak{k}}\left(x_{n} \cdots x_{2} x_{1}\right) .
$$


Si tous les $X_{i}$ sont dans $\mathfrak{k}$ on trouve

$$
\operatorname{tr}_{\mathfrak{p}}\left(x_{1} \cdots x_{n}\right)+(-1)^{n-1} \operatorname{tr}_{\mathfrak{k}}\left(x_{1} x_{n} \cdots x_{2}\right)=\operatorname{tr}_{\mathfrak{p}}\left(x_{1} \cdots x_{n}\right)+(-1)^{n-1} \operatorname{tr}_{\mathfrak{k}}\left(x_{n} \cdots x_{2} x_{1}\right) .
$$

\subsection{Cas résoluble}

Proposition 8. - Quand $(\mathfrak{g}, \sigma)$ est une paire symétrique résoluble on a $E=1$. Plus généralement quand $X$ est dans un idéal résoluble on a $E(X, Y)=1$ pour tout $Y \in \mathfrak{p}$.

Corollaire 5. - Pour toute paire symétrique $(\mathfrak{g}, \sigma)$, on a $\underset{\mathrm{CF}}{\star} g=f g$ pour $f, g \in S(\mathfrak{p})^{\mathfrak{k}}$ dès que $f \in S(J)^{\mathfrak{k}}$ où $J$ est idéal $\sigma$-stable et résoluble de $\mathfrak{g}$.

Démonstration. - En effet tous les crochets en $X, Y$ sont unipotents. Donc il n'y a que des roues pures, nécessairement de taille $2 n$. On a donc des termes de la forme (avec $x_{i}=\operatorname{ad} X$ ou $\operatorname{ad} Y$ )

$$
w_{\Gamma}\left(\operatorname{tr}_{\mathfrak{p}}\left(x_{1} \ldots x_{2 n}\right)-\operatorname{tr}_{\mathfrak{k}}\left(x_{1} \ldots x_{2 n}\right)\right) .
$$

Cette expression est nulle car on peut permuter les termes deux à deux (ça fait apparaître un élément unipotent), et on peut changer la trace sur $\mathfrak{k}$ en une trace sur $\mathfrak{p}$ pour la même raison.

On peut appliquer la proposition au cas où $X= \pm Y$ et on obtient

$$
E(X, X)=E(X,-X)=1 .
$$

On montrera au théorème 4 (§4.1) que le produit $\underset{\mathrm{CF}}{\star}$ et le produit de Rouvière coïncident $\operatorname{sur} S(\mathfrak{p})^{\mathfrak{k}}$. On déduit de la proposition précédente une autre preuve du résultat fondamental de Rouvière [25] :

THÉORÈme 2 ([25], Théorème 5.1). - La formule de Rouvière est un isomorphisme pour la convolution des germes de distributions $K$-invariantes sur l'espace symétrique résoluble $G / K$.

\subsection{Propriétés supplémentaires}

Lemme 13. - La fonction $E(X, Y)$ ne dépend que d'une composante réductive $\mathfrak{r}$ de $\mathfrak{g}$. Cette fonction est la même que pour la paire symétrique dégénérée $\hat{\mathfrak{g}}=\mathfrak{r} \ltimes\left(\mathfrak{g}_{u}\right)_{a b}$.

Corollaire 6. - Le produit $\underset{\mathrm{CF}}{\star}$ ne fait pas intervenir de dérivées d'éléments unipotents.

Une autre conséquence de la proposition 7 est que $\ln (E(X, Y))$ s'écrit $\operatorname{tr}_{\mathfrak{p}}(A)$ où $A$ est dans l'idéal bilatère $\mathcal{I}$ engendré par ad $[X, Y]=x y-y x .{ }^{(33)}$ En effet considérons une roue $\Gamma$ attachée aux mots $X_{1}, \ldots, X_{n}$. Si l'un des mots $X_{i}$ est de longueur plus grande que 1 , alors $A=\operatorname{ad} X_{1} \operatorname{ad} X_{2} \ldots \operatorname{ad} X_{n} \in \mathcal{I}$. Si tous les $X_{i}$ valent $X$ ou $Y$ alors, d'après la proposition 7 , les contributions se regroupent sous la forme

$$
w_{\Gamma}\left(\operatorname{tr}_{\mathfrak{p}}\left(x_{1} \ldots x_{2 n}\right)-\operatorname{tr}_{\mathfrak{k}}\left(x_{1} \ldots x_{2 n}\right)\right)=w_{\Gamma}\left(\operatorname{tr}_{\mathfrak{p}}\left(x_{1} \ldots x_{2 n}\right)-\operatorname{tr}_{\mathfrak{p}}\left(x_{2 n} x_{1} \ldots x_{2 n-1}\right)\right) .
$$

On écrit $\operatorname{tr}_{\mathfrak{p}}\left(x_{1} \ldots x_{2 n}\right)=\operatorname{tr}_{\mathfrak{p}}\left(x_{2} x_{1} x_{3} \ldots x_{2 n}\right)+\operatorname{tr}_{\mathfrak{p}}\left(\left[x_{1}, x_{2}\right] x_{3} \ldots x_{2 n}\right)$, ce qui montre que, modulo des termes de la forme $\operatorname{tr}_{\mathfrak{p}}(\mathcal{I})$, on $\operatorname{atr}_{\mathfrak{p}}\left(x_{1} \ldots x_{2 n}\right)=\operatorname{tr}_{\mathfrak{p}}\left(x_{2 n} x_{1} \ldots x_{2 n-1}\right)$. On retrouve ainsi le résultat de [25] théorème 3.15 .

(33) Ce genre de résultat devrait s'énoncer plus correctement dans l'algèbre libre engendrée par $\operatorname{ad} X, \operatorname{ad} Y$. 


\subsection{Calculs numériques à l'ordre 4 pour $\ln \left(E_{\lambda}(X, Y)\right)$}

Composante surk $\mathfrak{k}$. - On calcule d'abord le poids des graphes qui vont intervenir à l'ordre 4 . Ces calculs se font en utilisant la formule de Stokes. On trouve le développement suivant :

$\frac{1}{2}[X, Y]+\frac{-1}{24}[X,[X,[X, Y]]]+\frac{-1}{24}[Y,[Y,[X, Y]]]+\frac{-1}{48}[X,[Y,[X, Y]]]+\frac{-1}{48}[Y,[X,[X, Y]]]$.

Lorsque le caractère $\lambda$ de la sous-algèbre $\mathfrak{k}$ vaut $\frac{1}{2} \operatorname{tr}_{\mathfrak{k}} \mathrm{ad}$, on peut écrire ce terme à l'ordre 4 sous la forme

$$
\frac{1}{4} \operatorname{tr}_{\mathfrak{k}}(x y-y x)+\frac{1}{12} \operatorname{tr}_{\mathfrak{k}}\left(y x^{3}-x^{3} y+y^{3} x-x y^{3}\right)+\frac{1}{24} \operatorname{tr}_{k}\left((y x)^{2}-(x y)^{2}\right),
$$

où $x=\operatorname{ad} X$ et $y=\operatorname{ad} Y$. C'est bien l'expression que l'on trouve dans [26] page 256.

Composante scalaire. - D'après le lemme 11, la fonction $\ln (E(X, Y)$ est paire. Les contributions à l'ordre 2 sont nulles, car d'après la proposition 7 elles sont de la forme $\operatorname{tr}_{\mathfrak{p}}(\operatorname{ad} X \operatorname{ad} Y)-$ $\operatorname{tr}_{\mathfrak{k}}(\operatorname{ad} Y \operatorname{ad} X)=0$.

Afin de comparer notre fonction $E(X, Y)$ à celle de Rouvière $e(X, Y)$, on regarde les contributions à l'ordre 4 en $X, Y$, en fonction de la taille des roues.

Lorsque la roue est de taille 2 , les arêtes sortantes sont colorées par $\mathfrak{k}^{*}$, sinon on aurait une arête double. On trouve, pour le terme de degré 4 en $X, Y$, une expression de la forme :

$$
w_{\Gamma}\left(\operatorname{tr}_{\mathfrak{p}}-\operatorname{tr}_{\mathfrak{k}}\right)(\operatorname{ad}[X, Y] \operatorname{ad}[X, Y])=w_{\Gamma} b([X, Y],[X, Y]),
$$

où $b(A, B)=K_{\mathfrak{g}}(A, B)-2 K_{\mathfrak{k}}(A, B)$ est la différence des formes de Killing sur $\mathfrak{g}$ et $\mathfrak{k}$, comme dans [26].

Pour les roues de taille 3 , les contributions à l'ordre 4 font intervenir 2 sorties dans $\mathfrak{p}^{*}$. Compte tenu des symétries, le seul cas non trivial concerne les sorties $[X, Y], X, Y$ (dessin de gauche de (3.7)). On trouve une contribution de la forme précédente $C_{3} b([X, Y],[X, Y])$.

Pour les roues de taille 4 , qui sont nécessairement des roues pures, les seules contributions non triviales pour le symbole font intervenir des sorties attachées sur $X, X, X, Y$ ou $Y, Y, Y, X$ (dessin de droite de (3.7)). Toutefois le coefficient associé est nul car on peut intervertir les positions 2 et 4 ce qui échange 3 groupes de 2 arêtes.

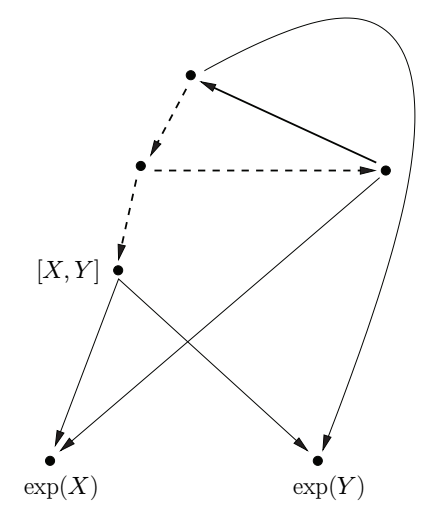

Roue taille 3 sur $[X, Y], X, Y$

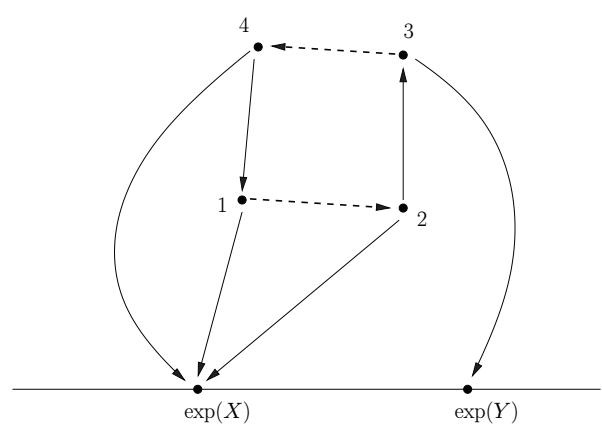

Roue pure taille 4 sur $X, X, X, Y$

On résume ces calculs par la proposition suivante : 
Proposition 9. - Jusqu'à l'ordre 4 au moins, la fonction $E_{\frac{1}{2} \operatorname{tr}_{k}}(X, Y)$ cö̈ncide avec la fonction $e(X, Y)$ de Rouvière.

Démonstration. - D'après les calculs précédents, les contributions scalaires à l'ordre 4 correspondent à un terme de la forme

$$
\exp \left(c\left(\operatorname{tr}_{\mathfrak{p}}-\operatorname{tr}_{\mathfrak{k}}\right)(\operatorname{ad}[X, Y])^{2}+\ldots\right) .
$$

On verra au théorème 4 (§4.1) que le produit de Cattaneo-Felder coïncide avec celui de Rouvière, ce qui montre que la constante $c$ est uniquement déterminée par un calcul explicite. On peut le faire dans $s l(2)$ et on trouve $c=1 / 240$, résultat conforme (au signe près) à celui de Rouvière ([27] page 257).

Voici le détail du calcul : $\widehat{H}, \widehat{X}, \widehat{Y}$ désigne la base de $\operatorname{sl}(2)$ et $\mathfrak{k}=\langle\widehat{X}-\widehat{Y}\rangle$ et $\mathfrak{p}=\langle\widehat{H}, \widehat{X}+\widehat{Y}\rangle$. On pose $\omega=\widehat{H}^{2}+(\widehat{X}+\widehat{Y})^{2}$ et $\Omega=\beta(\omega)$. On a pour $X \in \mathfrak{p}$

$$
J^{1 / 2}(X)=1+\frac{1}{12} \operatorname{tr}_{\mathfrak{p}}(\operatorname{ad} X)^{2}+\frac{1}{360} \operatorname{tr}_{\mathfrak{p}}(\operatorname{ad} X)^{4}+\cdots
$$

On trouve $\partial_{J^{1 / 2}}\left(\omega^{2}\right)=\omega^{2}+\frac{16}{3} \omega+\frac{128}{45}$ et $\beta\left(\omega^{2}\right)=\Omega^{2}-\frac{8}{3} \Omega(\bmod U(\mathfrak{g}) \cdot \mathfrak{k})$. Modulo $U(\mathfrak{g}) \cdot \mathfrak{k}$ on a donc

$$
\partial_{J^{1 / 2}}\left(\omega^{2}\right) \cdot \partial_{J^{1 / 2}}\left(\omega^{2}\right)=\Omega^{2}+\frac{8}{3} \Omega+\frac{16}{3}=\beta\left(\partial_{J^{1 / 2}}\left(\omega^{2}-\frac{16}{15}\right)\right) .
$$

Pour $\operatorname{sl}(2)$ on calcule $\left(\operatorname{tr}_{\mathfrak{p}}-\operatorname{tr}_{\mathfrak{k}}\right)(\operatorname{ad}[X, Y])^{2}$ agissant comme opérateur bidifférentiel sur $\omega \otimes \omega$. On trouve -256 , la constante vaut donc $\frac{16}{15 \times 256}=\frac{1}{240}$.

\subsection{Cas quadratique}

On dit qu'une paire symétrique $(\mathfrak{g}, \sigma)$ est quadratique si elle est munie d'une forme bilinéaire invariante et non dégénérée (mais on ne suppose pas que c'est la forme de Killing!).

Cas des paires d'Alekseev-Meinrenken. - C'est par définition le cas des paires quadratiques pour lesquelles la forme bilinéaire est $\sigma$-anti-invariante. On a alors les identifications $\mathfrak{p}^{*} \sim \mathfrak{k}$ et $\mathfrak{k}^{*} \sim \mathfrak{p}$. En utilisant la proposition 7 on trouve :

$$
\begin{aligned}
& \operatorname{tr}_{\mathfrak{p}}\left(x_{1} \cdots x_{n}\right)+(-1)^{n-1} \operatorname{tr}_{\mathfrak{k}}\left(x_{n} \cdots x_{1}\right) \\
& \quad=\operatorname{tr}_{\mathfrak{p}}\left(x_{1} \cdots x_{n}\right)+(-1)^{n-1} \operatorname{tr}_{\mathfrak{p}^{*}}\left(x_{n} \cdots x_{1}\right)=\operatorname{tr}_{\mathfrak{p}}\left(x_{1} \cdots x_{n}\right)-\operatorname{tr}_{\mathfrak{p}}\left(x_{1} \cdots x_{n}\right)=0 .
\end{aligned}
$$

On en déduit le résultat important suivant :

Proposition 10. - Pour une paire symétrique d'Alekseev-Meinrenken la fonction E vaut 1 .

Dans le théorème 4, comme déjà annoncé, on fera le lien entre la fonction $E$ et le produit de Rouvière, ce qui entraînera notamment que notre fonction $E$ décrit la convolution des distributions $K$-invariantes sur l'espace symétrique $G / K$. Par conséquent on retrouve un résultat démontré par Alekseev-Meinrenken [1] et Torossian [35], à savoir que la symétrisation, modifiée par la racine carrée du jacobien, est un isomorphisme d'algèbres dans le cas des paires symétriques avec forme $\sigma$-anti-invariante. Dans notre situation on démontre une conjecture formulée dans [35] : cet isomorphisme s'étend aux germes de distributions invariantes car la fonction $E(X, Y)$ vaut 1 . On résume la situation par le théorème suivant. 
ThÉorème 3. - Pour une paire symétrique d' Alekseev-Meinrenken, la formule de Rouvière est encore un isomorphisme au niveau des germes de distributions $K$-invariantes.

Cas quadratique $\sigma$-invariant. - C'est par définition le cas des paires quadratiques pour lesquelles la forme bilinéaire est $\sigma$-invariante. On a alors les identifications $\mathfrak{p} \sim \mathfrak{p}^{*}$ et $\mathfrak{k} \sim \mathfrak{k}^{*}$ et l'endomorphisme transposé vérifie ${ }^{t} \operatorname{ad} X=-\operatorname{ad} X$. On a donc :

$$
\begin{aligned}
\operatorname{tr}_{\mathfrak{p}}\left(x_{1} \cdots x_{n}\right)+(-1)^{n-1} \operatorname{tr}_{\mathfrak{k}}\left(x_{n} \cdots x_{1}\right)= \\
\quad \operatorname{tr}_{\mathfrak{p}}\left(x_{1} \cdots x_{n}\right)+(-1)^{n-1} \operatorname{tr}_{\mathfrak{k}^{*}}\left(x_{n} \cdots x_{1}\right)=\operatorname{tr}_{\mathfrak{p}}\left(x_{1} \cdots x_{n}\right)-\operatorname{tr}_{\mathfrak{k}}\left(x_{1} \cdots x_{n}\right)
\end{aligned}
$$

Si de plus, la paire symétrique est très-symétrique ${ }^{(34)}$, alors par un calcul élémentaire en termes de matrices blocs $2 \times 2$, on trouve :

$$
\operatorname{tr}_{\mathfrak{p}}\left(x_{1} \cdots x_{n}\right)-\operatorname{tr}_{\mathfrak{k}}\left(x_{1} \cdots x_{n}\right)=0 .
$$

On en déduit la proposition suivante.

Proposition 11. - Si $(\mathfrak{g}, \sigma)$ est une paire très-symétrique et quadratique avec forme $\sigma$-invariante, alors la fonction $E$ vaut 1.

Par exemple une algèbre de Lie considérée comme un espace symétrique est une paire trèssymétrique, car on a $\sigma(x, y)=(y, x)$ et $A(x, y)=(x,-y)$. Pour une paire symétrique complexe, on peut prendre pour $A$ la multiplication par $i$. La proposition ci-dessus s'applique notamment dans le cas des algèbres de Lie quadratiques vues comme des paires symétriques $\mathfrak{g} \times \mathfrak{g} /$ diagonale.

Corollaire 7. - Si $\mathfrak{g}$ est une algèbre de Lie quadratique vue comme une paire symétrique $\mathfrak{g} \times \mathfrak{g} /$ diagonale, alors la fonction $E$ vaut 1 .

Ce corollaire nous suggère la conjecture suivante.

Conjecture 1. - Pour les algèbres de Lie, vues comme paires symétriques $\mathfrak{g} \times \mathfrak{g} /$ diagonale, la fonction $E$ vaut 1 .

\section{4. Écriture en coordonnées exponentielles des opérateurs différentiels invariants sur un espace symétrique}

On considère $(\mathfrak{g}, \sigma)$ une paire symétrique. Dans le diagramme de Cattaneo-Felder pour la bi-quantification, on considère le couple de variétés co-isotropes $C_{2}=\mathfrak{k}^{\perp}$, mis en position verticale, et $C_{1}=0^{\perp}=\mathfrak{g}^{*}$, mis en position horizontale ( $c f$. § 1.6). Comme dans [19] on considère pour le bi-vecteur de Poisson, la moitié du crochet de Lie. Alors $(U(\mathfrak{g}), \cdot)$ est isomorphe à $(S(\mathfrak{g}), \underset{\mathrm{DK}}{\star})$.

(34) Une paire symétrique est dite très-symétrique s'il existe un opérateur $A \operatorname{sur} \mathfrak{g}$ tel que $A \circ \operatorname{ad} X=\operatorname{ad} X \circ A$ avec $A: \mathfrak{k} \longrightarrow \mathfrak{p}$ et $\mathfrak{p} \longrightarrow \mathfrak{k}$.

$4^{\mathrm{e}}$ SÉRIE - TOME $41-2008-\mathrm{N}^{\mathrm{o}} 5$ 


\subsection{Liens entre les produits de Rouvière et de Cattaneo-Felder}

Produit de Duflo-Kontsevich. - Rappelons que le produit de Duflo-Kontsevich sur $S(\mathfrak{g})$ est donné par la formule

$$
\beta\left(\partial_{q^{1 / 2}}(f \underset{\mathrm{DK}}{\star} g)\right)=\beta\left(\partial_{q^{1 / 2}} f\right) \cdot \beta\left(\partial_{q^{1 / 2}} g\right),
$$

où $q$ désigne la fonction

$$
q(X)=\operatorname{det}_{\mathfrak{g}}\left(\frac{\sinh \frac{\operatorname{ad} X}{2}}{\frac{\operatorname{ad} X}{2}}\right) .
$$

Roues horizontales et verticales. - L'espace de réduction, pour la partie verticale, est l'algèbre $S(\mathfrak{p})^{\mathfrak{k}}$ munie du produit $\underset{\mathrm{CF}}{\star}$. L'espace de réduction, pour la partie horizontale, est l'algèbre $S(\mathfrak{g})$ munie du produit de Duflo-Kontsevich.

Pour $f \in S(\mathfrak{g})$, notons $A(f)$ les contributions sur l'axe horizontal $\mathfrak{g}^{*}(c f$. Fig. 5)

$$
1 \star f=A(f) .
$$

Alors $A$ est un opérateur à valeurs dans $S(\mathfrak{p})$, donné par des graphes de Kontsevich à 4 couleurs, qui ne se réduisent pas aux roues pures. Cet opérateur est compliqué et n'est pas à coefficients constants car les graphes avec arêtes doubles colorées par ++ et +- ne sont pas nuls a priori.
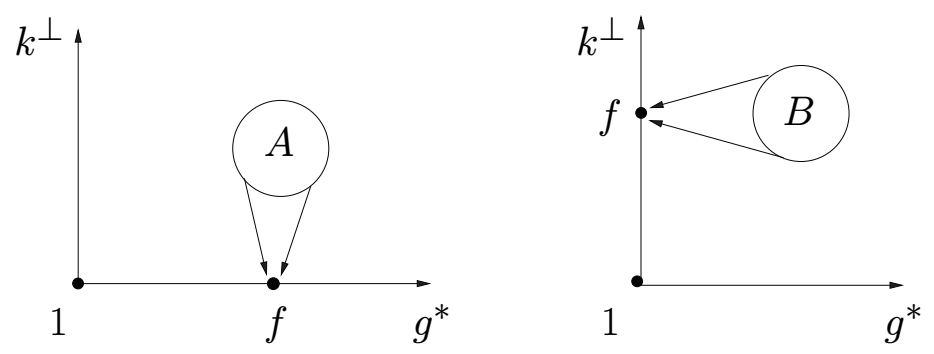

FIgURE 5. Contributions des roues pures sur les axes

En fait, l'opérateur $A$ correspond à une modification de la projection de $S(\mathfrak{g})$ sur $S(\mathfrak{p})$ via la symétrisation, c'est-à-dire la décomposition $U(\mathfrak{g})=\beta\left(\partial_{q^{1 / 2}} S(\mathfrak{p})\right) \oplus \mathfrak{k} \cdot U(\mathfrak{g})$. En effet, lorsqu'on munit $S(\mathfrak{g})$ du produit de Duflo-Kontsevich, on a $S(\mathfrak{g})=S(\mathfrak{p}) \oplus \underset{\text { DK }}{\star} S(\mathfrak{g})$ et donc, grâce à l'application $\beta \circ \partial_{q^{1 / 2}}$, on a :

$$
U(\mathfrak{g})=\beta \circ \partial_{q^{1 / 2}}(S(\mathfrak{p})) \oplus \mathfrak{k} \cdot U(\mathfrak{g}) .
$$

En particulier on a l'équivalence

$$
1 \star f=0 \Longleftrightarrow f \in \underset{1}{k}{ }_{1} S(\mathfrak{g}) .
$$

Lorsque $f$ est dans $S(\mathfrak{p})$, l'opérateur $A$ correspond aux contributions des roues pures; c'est un opérateur à coefficients constants que l'on notera encore $A$. 
Notons $B(f)$ les contributions sur l'axe vertical $\mathfrak{k}^{\perp}$; c'est un opérateur à coefficients constants correspondant aux contributions des roues pures attachées sur $\mathfrak{k}^{\perp}$ :

$$
f \underset{2}{\star 1}=B(f)
$$

On notera $A(X)$ et $B(X)$ les symboles associés. On a donc pour $X \in \mathfrak{p}$,

$$
A(X)=\left(1 \underset{1}{\star} e^{X}\right) e^{-X} \quad \text { et } B(X)=\left(e_{2}^{X} \star 1\right) e^{-X} .
$$

Ce sont des symboles inversibles et $\mathfrak{k}$-invariants.

Comparaison des star-produits $\sharp_{R o u}$ et $\star_{C F}$. - Soient $f, g \in S(\mathfrak{p})^{\mathfrak{k}}$. Par compatibilité en cohomologie des produits $\underset{1}{\star}$ et $\underset{2}{\star}$, on a $\underset{2}{\star} \underset{1}{\star} 1=1 \underset{1}{\star} A^{-1} B(f)$ et par suite

$$
(f \underset{\mathrm{CF}}{\star} g) \underset{2}{\star} 1=1 \underset{1}{\star} A^{-1} B(f \underset{\mathrm{CF}}{\star} g),
$$

puis

$$
(f \underset{\mathrm{CF}}{\star} g) \underset{2}{\star} 1=1 \underset{1}{\star}\left(A^{-1} B(f) \underset{\mathrm{DK}}{\star} A^{-1} B(g)\right) .
$$

On en déduit que l'on a

$$
A^{-1} B(f \underset{\mathrm{CF}}{\star} g)=A^{-1} B(f) \underset{\mathrm{DK}}{\star} A^{-1} B(g) \text { modulo } \underset{\mathrm{DK}}{\mathfrak{k}} S(\mathfrak{g}) .
$$

Comme $\beta \circ \partial_{q^{1 / 2}}$ transforme le produit de Duflo en le produit dans l'algèbre enveloppante, on en déduit que

$$
\beta \circ \partial_{q^{1 / 2}} \circ A^{-1} B
$$

transforme le produit $\underset{\mathrm{CF}}{\star}$ en le produit dans l'algèbre enveloppante modulo $\mathfrak{k} \cdot U(\mathfrak{g})$, c'est-àdire que l'on a modulo $\mathfrak{k} \cdot U(\mathfrak{g})$ :

$$
\left(\beta \circ \partial_{q^{1 / 2}} \circ A^{-1} B\right)(f \underset{\mathrm{CF}}{\star} g)=\left(\beta \circ \partial_{q^{1 / 2}} \circ A^{-1} B\right)(f)\left(\beta \circ \partial_{q^{1 / 2}} \circ A^{-1} B\right)(g)
$$

Soit $J(X)=\operatorname{det}_{\mathfrak{p}}\left(\frac{\sinh (\operatorname{ad} X)}{\operatorname{ad} X}\right)$. Remarquons que, pour $X \in \mathfrak{p}$, on a $q^{1 / 2}(X)=J\left(\frac{X}{2}\right)$. Le produit de Rouvière [25] est défini par la formule, pour $p, q \in S(\mathfrak{p})$ :

$$
\beta\left(\partial_{J^{\frac{1}{2}}}(p \underset{\text { Rou }}{\sharp q)})\right)=\beta\left(\partial_{J^{\frac{1}{2}}}(p)\right) \cdot \beta\left(\partial_{J^{\frac{1}{2}}}(p)\right) \text { modulo } \mathfrak{k} \cdot U(\mathfrak{g}) .
$$

On va voir que les deux produits coïncident sur les éléments k-invariants.

ThÉORÈme 4. - Les produits de Rouvière et de Cattaneo-Felder coïncident pour toute paire symétrique. Pour $f$ et $g$ dans $S(\mathfrak{p})^{\mathfrak{k}}$, on a

$$
\beta\left(\partial_{J^{\frac{1}{2}}}(f \underset{\mathrm{CF}}{\star} g)\right)=\beta\left(\partial_{J^{\frac{1}{2}}}(f)\right) \cdot \beta\left(\partial_{J^{\frac{1}{2}}}(g)\right) \text { modulo } \mathfrak{k} \cdot U(\mathfrak{g}) .
$$

Preuve du théorème. - La preuve se fait comme dans [19]. On compare deux isomorphismes. On démontre un lemme et une formule intermédiaires.

Lemme 14. - Il existe des algèbres de Lie résolubles pour lesquelles $\operatorname{tr}_{\mathfrak{p}}(\operatorname{ad} X)^{2 n}$ n'agit pas comme une dérivation sur $S(\mathfrak{p})^{\mathfrak{k}}$. 
Preuve du lemme. - Soit $\mathfrak{g}$ l'algèbre de Lie résoluble engendrée par $t, x, y, z$ et les relations

$$
[x, y]=z, \quad[t, x]=-x, \quad[t, y]=y .
$$

C'est une paire symétrique pour l'involution $\sigma(t)=-t, \quad \sigma(x)=y, \quad \sigma(z)=-z$. Le sousespace $\mathfrak{p}$ est engendré par $\langle t, x-y, z\rangle$ et le sous-espace $\mathfrak{k}$ est engendré par $\langle x+y\rangle$. On vérifie facilement que l'on a $S(\mathfrak{p})^{\mathfrak{k}}=\mathbb{C}\left[z, 4 z t+(x-y)^{2}\right]$. La forme $t^{*}$ est clairement $\mathfrak{k}$-invariante car on a $t^{*}([\mathfrak{k}, \mathfrak{p}])=0$. Par ailleurs l'application polynomiale $X \mapsto \operatorname{tr}_{\mathfrak{p}}(\operatorname{ad} X)^{2 n}$ s'identifie à $\left(t^{*}\right)^{2 n}$, qui n'agit pas comme une dérivation dans $S(\mathfrak{p})^{\mathfrak{k}}$.

Proposition 12. - On a l'égalité de fonctions universelles suivante pour $X \in \mathfrak{p}$,

$$
A(X) J^{\frac{1}{2}}(X)=B(X) q^{\frac{1}{2}}(X) .
$$

Preuve de la proposition. - Les fonctions $A, B, q, J$ ne font intervenir que les $\operatorname{tr}_{\mathfrak{p}}(\operatorname{ad} X)^{2 n}$ et sont universelles. Lorsque $\mathfrak{g}$ est résoluble, on sait que l'on a les égalités $E=e=1$ donc le produit de Rouvière et le produit $\underset{C F}{\star}$ correspondent au produit standard. Raisonnons par l'absurde. On disposerait d'un automorphisme non trivial pour le produit standard dans $S(\mathfrak{p})^{\mathfrak{k}}$ donné par l'exponentielle d'une série en $\operatorname{tr}_{\mathfrak{p}}(\operatorname{ad} X)^{2 n}$. On en déduirait qu'il existerait $n>0$ tel que $\operatorname{tr}_{\mathfrak{p}}(\operatorname{ad} X)^{2 n}$ agirait comme une dérivation sur $S(\mathfrak{p})^{\mathfrak{k}}$ ce qui n'est pas vrai d'après le lemme ci-dessus.

(Fin de la preuve du théorème) Grâce à la proposition 12 et à l'équation (4.6) le produit $\stackrel{\star}{\mathrm{CF}}$ et le produit de Rouvière coïncident sur les éléments $\mathfrak{k}$-invariants.

Remarque 7. - Pour $X \in \mathfrak{p}$ on a $q^{\frac{1}{2}}(X)=J\left(\frac{X}{2}\right)$ et on montrera dans la proposition 23 (§6.3) que l'on a $B(X)=1$. On en déduit la formule suivante pour $A(X)$

$$
A^{-1}(X) q^{\frac{1}{2}}(X)=A^{-1}(X) J\left(\frac{X}{2}\right)=J^{\frac{1}{2}}(X) .
$$

Remarque 8. - En inversant les rôles de $\mathfrak{g}^{*}$ et $\mathfrak{k}^{\perp}$ on montre de même, pour $f, g \in S(\mathfrak{p})^{\mathfrak{k}}$ :

$$
\beta\left(\partial_{J^{\frac{1}{2}}}(f \underset{\mathrm{CF}}{\star} g)\right)=\beta\left(\partial_{J^{\frac{1}{2}}}(f)\right) \cdot \beta\left(\partial_{J^{\frac{1}{2}}}(g)\right) \text { modulo } U(\mathfrak{g}) \cdot \mathfrak{k} .
$$

\subsection{Cas à paramètre}

Commutativité d'algèbres d'opérateurs différentiels invariants sur les $z$-densités. - Soit $\lambda$ un caractère de $\mathfrak{k}$ et notons $\mathfrak{k}^{-\lambda}$ le sous-espace de $S(\mathfrak{g})$ défini par $\{X-\lambda(X), X \in \mathfrak{k}\}$. On met la sous-variété co-isotrope $\lambda+\mathfrak{k}^{\perp}$ en position verticale et $\mathfrak{g}^{*}$ en position horizontale. On a $1 \star{ }_{1} K=\lambda(K)$, donc pour $f$ et $g$ deux éléments $\mathfrak{k}$-invariants, on a :

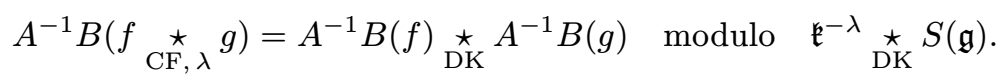

Action de la symétrie par rapport à la diagonale : Faisons agir $s$ la symétrie par rapport à la première diagonale. Les fonctions d'angle vérifient les relations suivantes :

$$
\begin{gathered}
\mathrm{d} \phi_{++}(p, q)=\mathrm{d} \phi_{--}(q, p) \\
\mathrm{d} \phi_{++}(s(p), s(q))=-\mathrm{d} \phi_{++}(p, q) \\
\mathrm{d} \phi_{+-}(s(p), s(q))=-\mathrm{d} \phi_{-+}(p, q) .
\end{gathered}
$$


Comme les roues pures $A$ et $B$ sont paires, l'action de la symétrie $s$ les préserve et nos notations restent cohérentes; $A$ est l'action des roues pures attachées sur $\mathfrak{g}^{*}$ que l'on choisisse la position horizontale ou verticale.

L'action de la symétrie revient à mettre en position horizontale la sous-variété $\lambda+\mathfrak{k}^{\perp}$ et en position verticale $\mathfrak{g}^{*}$.

Considérons deux fonctions $f, g$ positionnées sur l'axe horizontal et regardons le produit limite quand les positions de $f$ et $g$ se rapprochent. Dans la première configuration limite ( $\mathfrak{g}^{*}$ en position horizontale) on retrouve le produit de Duflo-Kontsevich $f_{\text {DK }}^{\star} g$. Par symétrie diagonale on trouvera dans la deuxième configuration limite ( $\mathfrak{g}^{*}$ en position verticale), $g_{\mathrm{DK}, \text { signes }} f$, les signes provenant de l'action de $s$ sur les fonctions d'angle. Or les coefficients avec fonction d'angle à une couleur (ou deux couleurs) ont une propriété de symétrie par rapport à l'axe vertical : $(-1)^{n} w_{\Gamma}=w_{\hat{\Gamma}}$. En tenant compte de cette symétrie on constate que $g_{\mathrm{DK}, \stackrel{\star}{\text { signes }}}^{\star} f$ vaut $f_{\mathrm{DK}}^{\star} g$.

Du côté du produit de Cattaneo-Felder pour le paramètre $\lambda$, lorsque $f$ et $g$ sont positionnées sur l'axe vertical, on trouvera dans la première configuration limite $\left(\lambda+\mathfrak{k}^{\perp}\right.$ en position verticale ) $f_{\mathrm{CF}, \lambda}^{\star} g$ et par symétrie dans l'autre configuration limite $\left(\lambda+\mathfrak{k}^{\perp}\right.$ en position horizontale) $g_{C F,-\lambda}^{\star} f$.

On en déduit les égalités, pour $f, g \in S(\mathfrak{p})^{\mathfrak{k}}$ :

$$
\begin{aligned}
A^{-1} B\left(f_{C F, \lambda}^{\star} g\right) & =A^{-1} B(f) \underset{\mathrm{DK}}{\star} A^{-1} B(g) & \text { modulo } & \mathfrak{k}^{-\lambda} \underset{\mathrm{DK}}{\star} S(\mathfrak{g}) \\
A^{-1} B\left(g_{\mathrm{CF},-\lambda}^{\star} f\right) & =A^{-1} B(f) \underset{\mathrm{DK}}{\star} A^{-1} B(g) & \text { modulo } & S(\mathfrak{g}) \underset{\mathrm{DK}}{\star} \mathfrak{k}^{-\lambda} .
\end{aligned}
$$

Or d'après le lemme 11 (§3.1) on a $f_{\mathrm{CF}, \lambda}^{\star} g=g_{\mathrm{CF},-\lambda}{ }_{\star}^{\star} f$ et d'après un résultat de Duflo [12], résultant de la dualité de Poincaré, on a :

$$
\mathfrak{k}^{-\lambda} \cdot U(\mathfrak{g}) \cap U(\mathfrak{g})^{\mathfrak{k}}=U(\mathfrak{g})^{\mathfrak{k}} \cap U(\mathfrak{g}) \cdot \mathfrak{k}^{-\lambda+\operatorname{tr}_{\mathfrak{k}}} .
$$

On en déduit le théorème suivant, qui étend le critère de commutativité des algèbres d'opérateurs différentiels sur les demi-densités ${ }^{(35)}$ et sur les fonctions ${ }^{(36)}$.

ThÉORÈMe 5. - Pour $f, g$ dans $S(\mathfrak{p})^{\mathfrak{k}}$ et pour tout $z \in \mathbb{R}$, on a la relation

$$
f_{\mathrm{CF}, \lambda}^{\star} g=f_{C F, \lambda+z \operatorname{tr}_{\mathfrak{k}}}^{\star} g .
$$

En particulier le produit naturel dans $\left(U(\mathfrak{g}) / U(\mathfrak{g}) \cdot \mathfrak{k}^{z \mathrm{tr}_{\mathfrak{k}}}\right)^{\mathfrak{k}}$, l'algèbre des opérateurs différentiels invariants sur les $z$-densités, est commutatif pour tout $z$.

Démonstration. - Les fonctions $E_{\lambda+\operatorname{tr}_{\mathfrak{e}}}$ et $E_{\lambda}$ définissent les mêmes star-produits sur les éléments ḱ-invariants d'après la relation de Duflo et les formules (4.10), (4.11). Il résulte par récurrence qu'il en est de même pour $E_{\lambda+n \operatorname{tr}_{\mathfrak{k}}}$ et par prolongement des identités polynomiales pour $E_{\lambda+z \operatorname{tr}_{\mathfrak{k}}}$.

(35) Cas $z=\frac{1}{2}$ [12], on utilise la relation $\frac{1}{2} \operatorname{tr}_{\mathfrak{k}}=-\frac{1}{2} \operatorname{tr}_{\mathfrak{g} / \mathfrak{k}}+\frac{1}{2} \operatorname{tr}_{\mathfrak{g}}$ et le fait que l'action du caractère $\operatorname{tr}_{\mathfrak{g}}$ n'est pas significative.

(36) Cas $z=0$ [21].

$4^{\mathrm{e}}$ SÉRIE - TOME $41-2008-\mathrm{N}^{\mathrm{o}} 5$ 
Comparaison des fonctions $E_{\lambda}(X, Y)$ et $e_{-\lambda}(X, Y)$. - On applique à l'équation (4.11) l'opérateur $\beta\left(\partial_{q^{1 / 2}}\right)$ qui transforme le produit $\underset{\mathrm{DK}}{\star}$ en le produit dans l'algèbre enveloppante. Compte tenu de (4.7), on trouve

$$
\beta\left(\partial_{J^{1 / 2}}\left(f_{\mathrm{CF}, \lambda}^{\star} g\right)\right)=\beta\left(\partial_{J^{1 / 2}} f\right) \cdot \beta\left(\partial_{J^{1 / 2}} g\right) \text { modulo } U(\mathfrak{g}) \cdot \mathfrak{k}^{-\lambda} .
$$

Les fonctions $e_{-\lambda}(X, Y)$ vérifient le même type de relation. On en déduit que $E_{\lambda}(X, Y)$ et $e_{-\lambda}(X, Y)$ doivent définir le même star-produit.

Or d'après [29], les fonctions $e_{-\lambda}(X, Y)$ ne sont symétriques que pour $-\lambda=\frac{1}{2} \operatorname{tr}_{\mathfrak{k}}$ et les premiers termes du développement calculé dans la proposition $9(\S 3.5)$ laissent à penser que l'on a l'égalité conjecturale $E_{\frac{1}{2} \operatorname{trk}_{\mathfrak{k}}}(X, Y) \stackrel{? ?}{=} e(X, Y)$. On devrait avoir aussi de manière conjecturale l'égalité $E_{\lambda}(X, Y) \stackrel{\text { ?? }}{=} e_{-\lambda+\frac{1}{2} \operatorname{tr}_{k}}(X, Y)$.

Conjecture 2. - On a $E_{\lambda}(X, Y)=e_{-\lambda+\frac{1}{2} \operatorname{tr}_{k}}(X, Y)$.

\subsection{Opérateurs différentiels invariants en coordonnées exponentielles}

On considère le couple de variétés co-isotropes $C_{1}=\mathfrak{k}^{\perp}$ et $C_{2}=0^{\perp}=\mathfrak{g}^{*}$. Le bi-vecteur de Poisson vaut la moitié du crochet de Lie et $(U(\mathfrak{g}), \cdot)$ est isomorphe à $(S(\mathfrak{g}), \underset{\mathrm{DK}}{\star})$.

Les espaces de réduction sont, pour la partie horizontale, l'algèbre $S(\mathfrak{p})^{\mathfrak{k}}$ munie du produit $\underset{\mathrm{CF}}{\star}=\underset{\text { Rou }}{\sharp}$ et, pour la partie verticale, l'algèbre $S(\mathfrak{g})$ munie du produit de Kontsevich. L'espace de réduction à l'origine est $S(\mathfrak{p})$, c'est-à-dire les distributions portées par l'origine sur l'espace symétrique $G / K$.

On en déduit pour $S(\mathfrak{p})$ l'existence d'une action à gauche de $(S(\mathfrak{g}), \underset{\text { Kont }}{\star})$ et d'une action à droite de $\left(S(\mathfrak{p})^{\mathfrak{k}}, \underset{\mathrm{CF}}{\star}\right)$.

Soit $R \in S(\mathfrak{p})^{\mathfrak{k}}$. On place dans le quadrant de Cattaneo-Felder, $e^{X}$ à l'origine et $R$ sur l'axe horizontal : on obtient donc $e^{X} \star R$. C'est donc un élément de $S(\mathfrak{p})$ qui dépend de $X$, que l'on voit comme un opérateur différentiel sur $\mathfrak{p}$ au point $X^{(37)}$. On a le résultat suivant qui résout de manière satisfaisante un problème posé par Duflo ([13] problème 7).

THÉORÈME 6. - La formule $e_{1}^{X} \underset{1}{ }$ R est l'écriture, en coordonnées exponentielles du conjugué par l'application $J^{1 / 2}(X)$ de l'opérateur différentiel invariant sur l'espace symétrique $G / K$, donné par $D_{\beta\left(J^{1 / 2}(\partial) R\right)}$.

Démonstration. - Soit $f \in \mathcal{C}^{\infty}(G / K)$ et $R \in S(\mathfrak{p})^{k}$. On a

$$
D_{\beta(R)} f(g K):=\left\langle R^{(Y)}, f(g \exp (Y) K)\right\rangle=\left.R\left(\partial_{Y}\right) f(g \exp (Y) K)\right|_{Y=0}
$$

où $R$ est vu comme une distribution de support $Y=0$. On note Exp la fonction $X \in \mathfrak{p} \mapsto$ $\exp _{G}(X) K \in G / K$ avec $X \in \mathfrak{p}$.

Si on tient compte du facteur $q^{1 / 2}$ entre $\mathcal{C}^{\infty}(G / K)$ et $\mathcal{C}^{\infty}(\mathfrak{p})$, on pose pour $X \in \mathfrak{p}$,

$$
\phi(X):=q^{1 / 2}(X) f\left(\exp _{G}(X) K\right)
$$

(37) Le terme $e^{X}$ est vu comme la distribution de Dirac au point $X$. 
Pour $u=\beta\left(\partial_{q^{1 / 2}} R\right)$, on note $D_{u}$ l'opérateur différentiel invariant à gauche sur l'espace symétrique associé à $u$. Il vient en écriture exponentielle

(4.12)

$$
D_{u}^{E x p}(\phi)(X):=q^{1 / 2}(X) D_{u}(f)\left(\exp _{G}(X) K\right)=\left\langle\left(\partial_{q^{1 / 2}} R\right)^{(Y)}, q^{1 / 2}(X)\left(\frac{\phi}{q^{1 / 2}}\right)(P(X, Y))\right\rangle,
$$

où on a écrit $\exp _{G}(X) \exp _{G}(Y)=\exp _{G}(P(X, Y)) \exp _{G}(K(X, Y))$ avec $P(X, Y) \in \mathfrak{p}$ et $K(X, Y) \in \mathfrak{k}$. Ces facteurs $P, K$ dépendent de manière analytique de $X, Y$ dans un voisinage de 0 . Le facteur $P(X, Y)$ est la série de Campbell-Hausdorff pour les espaces symétriques.

Rappelons que le produit de Kontsevich-Duflo est donné par la formule (4.1). Pour $X, Y$ dans $\mathfrak{p}$, on peut donc écrire :

$$
e^{X} \underset{\mathrm{DK}}{\star} e^{Y}=\frac{D(X, Y)}{D(P, K)} e^{P} \underset{\mathrm{DK}}{\star} e^{K},
$$

avec $D(X, Y)=\frac{q^{1 / 2}(X) q^{1 / 2}(Y)}{q^{1 / 2}(Z(X, Y))}$ la fonction de densité sur les algèbres de Lie.

On a $e_{2}^{K} \underset{2}{\star} 1=C(K)$, où $C(K)$ désigne les contributions des roues pures attachées à l'axe vertical avec rayons colorés par la couleur -+ . Comme $e^{K} \underset{\mathrm{DK}}{\star} e^{-K}=q(K)$ on doit avoir $e^{K} \underset{2}{\star}\left(e^{-K} \underset{2}{\star} 1\right)=C(K) C(-K)=q(K)$. On a $K(X, Y)=-K(Y, X)$, donc la partie symétrique de $C(K)$ vaut $q^{1 / 2}(K)$. En fait on a le résultat intermédiaire suivant.

Lemme 15. - La fonction $C$ est paire et vaut $q^{1 / 2}(K)$.

Preuve du lemme. - On a

$$
\left(e^{K / 2} \underset{\mathrm{DK}}{\star} e^{K / 2}\right) \underset{2}{\star} 1=\frac{q(K / 2)}{q^{1 / 2}(K)} e_{2}^{K} \underset{2}{\star 1}=C(K) \frac{q(K / 2)}{q^{1 / 2}(K)} .
$$

Par ailleurs on a aussi

$$
\left(e^{K / 2} \underset{\mathrm{DK}}{\star} e^{K / 2}\right) \underset{2}{\star} 1=e_{2}^{K / 2} \underset{2}{\star}\left(e^{K / 2} \star 1\right)=C(K / 2)^{2},
$$

d'où l'on tire

puis par récurrence

$$
\left(\frac{C(K / 2)}{q^{1 / 2}(K / 2)}\right)^{2}=\frac{C(K)}{q^{1 / 2}(K)},
$$

$$
\left(\frac{C\left(\frac{K}{2^{n}}\right)}{q^{1 / 2}\left(\frac{K}{2^{n}}\right)}\right)^{2^{n}}=\frac{C(K)}{q^{1 / 2}(K)} .
$$

Comme les fonctions $C$ et $q^{1 / 2}$ s'écrivent $\exp \left(\sum_{n \geq 2} w_{n} \operatorname{tr}_{\mathfrak{p}}(\operatorname{ad} K)^{n}+\sum_{n \geq 2} w_{n}^{\prime} \operatorname{tr}_{\mathfrak{k}}(\operatorname{ad} K)^{n}\right)$, on déduit que l'on a $\frac{C(K)}{q^{1 / 2}(K)}=\lim _{n \mapsto \infty}\left(\frac{C\left(\frac{K}{2^{n}}\right)}{q^{1 / 2}\left(\frac{K}{2^{n}}\right)}\right)^{2^{n}}=1$.

(Fin de la preuve du théorème :) On note, comme au §4.1, $A$ l'opérateur vertical (correspondant à $\mathfrak{g}^{*}$ ) des contributions des roues pures et $B$ l'opérateur horizontal ${ }^{(38)}$. Grâce au lemme ci-dessus, on déduit que l'on a

$$
\left(e^{X} \underset{\mathrm{DK}}{\star} e^{Y}\right) \underset{2}{\star} 1=\frac{D(X, Y)}{D(P, K)} e_{2}^{P} \underset{2}{\star}\left(e_{2}^{K} \underset{2}{\star} 1\right)=q^{1 / 2}(X) q^{1 / 2}(Y) \frac{A(P)}{q^{1 / 2}(P)} e^{P} .
$$

(38) Les axes sont inversés par rapport à la situation $\S 4.1$ et $B=1$ d'après la proposition 23 (§6.3). 
D'après la proposition 12, on a pour $X \in \mathfrak{p}, A(X) J^{\frac{1}{2}}(X)=B(X) q^{\frac{1}{2}}(X)$, d'où l'on tire l'égalité

$$
\left(e^{X} \underset{\mathrm{DK}}{\star} e^{Y}\right) \underset{2}{\star} 1=q^{1 / 2}(X) q^{1 / 2}(Y) \frac{B(P)}{J^{1 / 2}(P)} e^{P} .
$$

Par ailleurs le terme de gauche de (4.13) peut s'écrire, compte tenu de la compatibilité des produits :

$$
A(Y) B^{-1}(Y) e_{2}^{X} \underset{1}{\star}\left(1 \star e^{Y}\right) .
$$

Il vient donc la formule

$$
e_{2}^{X} \underset{1}{\star}\left(1 \underset{1}{\star} e^{Y}\right)=J^{1 / 2}(Y) q^{1 / 2}(X) \frac{B(P)}{J^{1 / 2}(P)} e^{P} .
$$

En différentiant par rapport à $Y$ selon le polynôme $R$, on peut remplacer $e^{Y}$ par $R$. On peut alors utiliser la ḱ-invariance de $R$ pour justifier de la compatibilité des produits $\underset{1}{\star}$ et $\underset{2}{\star}$. Il vient alors

$$
e^{X} \underset{2}{\star}(\underset{1}{\star} R)=R^{(Y)} J^{1 / 2}(Y) q^{1 / 2}(X) \frac{B(P)}{J^{1 / 2}(P)} e^{P(X, Y)}=\left(e^{X} \underset{2}{1}\right) \underset{1}{\star} R=A(X) e^{X} \star{ }_{1} R,
$$

c'est-à-dire, compte tenu de la relation $A J^{\frac{1}{2}}=B q^{\frac{1}{2}}$,

$$
e_{1}^{X}{ }_{1} R=\left.R\left(\partial_{Y}\right) \frac{J^{1 / 2}(Y) J^{1 / 2}(X)}{B(X)} \frac{B(P)}{J^{1 / 2}(P)} e^{P}\right|_{Y=0} .
$$

Cette formule est précisément l'écriture de l'opérateur $D_{\beta\left(J^{1 / 2}(\partial) R\right)}$ en coordonnées exponentielles sur l'espace symétrique $G / K$, modifié par la fonction $J^{1 / 2}(X) / B(X)$. Compte tenu du résultat maintes fois annoncé $B=1$, le théorème est démontré.

\subsection{Déformation de la formule de Baker-Campbell-Hausdorff pour les paires symétriques}

L'application Exp définit un difféomorphisme local de $\mathfrak{p}$ sur $G / K$. On en déduit alors l'existence d'une formule de Campbell-Hausdorff pour les espaces symétriques, définie de la manière suivante. Pour $X, Y \in \mathfrak{p}$ proche de 0 , il existe une série convergente $Z_{\text {sym }}(X, Y)$ à valeurs dans $\mathfrak{p}$ telle que l'on ait

$$
\exp _{G}(X) \operatorname{Exp}(Y)=\operatorname{Exp}\left(Z_{\text {sym }}(X, Y)\right) .
$$

C'est le facteur $P(X, Y)$ introduit dans la section précédente. En utilisant l'involution $\sigma$ on trouve facilement

$$
\exp _{G}\left(2 Z_{\text {sym }}(X, Y)\right)=\exp _{G}(X) \exp _{G}(2 Y) \exp _{G}(X) .
$$

Dans [17] Kashiwara-Vergne ont conjecturé que la déformation par dilatation de la formule de Campbell-Hausdorff était gouvernée par les champs adjoints. Cette conjecture a été démontrée dans [2] comme conséquence de [33] et de la quantification par déformation. Dans [25] F. Rouvière propose de faire de même pour la série $Z_{\text {sym }}(X, Y)$ en utilisant les champs $\mathfrak{k}$-adjoints. Dans [34] une approche via la quantification de Kontsevich fut proposée.

Dans cette section nous montrons qu'une déformation par les champs $\mathfrak{k}$-adjoints vient naturellement grâce au diagramme de bi-quantification. 
Première déformation. - Considérons le diagramme de bi-quantification de la section précédente, en mettant $\mathfrak{g}^{*}$ en position verticale et $\mathfrak{k}^{\perp}$ en position horizontale. Considérons $X, Y \in \mathfrak{p}$ et mettons les fonctions $e^{X}$ et $e^{Y}$ sur l'axe vertical comme précédemment.

La contribution des graphes colorés est indépendante de la position de $e^{X}$ et $e^{Y}$ sur l'axe vertical. En position limite, quand $e^{X}$ et $e^{Y}$ se rapprochent, on trouve d'après l'équation (4.13) et $B=1$ (Proposition $23(\S 6.3)$ ) :

$$
\left(e^{X} \star_{D K} e^{Y}\right) \star_{2} 1=\frac{q^{1 / 2}(X) q^{1 / 2}(Y)}{J^{1 / 2}\left(Z_{\mathrm{sym}}(X, Y)\right)} e^{Z_{\mathrm{sym}}(X, Y)} .
$$

Quand $Y$ tend vers l'origine, on trouve

$$
A(Y) e^{X} \star_{2} e^{Y}
$$

Ces deux expressions sont donc égales. Mettons maintenant $e^{X}$ sur l'axe vertical en position 1 et $e^{Y}$ sur l'axe horizontal en position $s$. Quand $s$ tend vers 0 on trouve

$$
e^{X} \star_{2}\left(1 \star_{1} e^{Y}\right)=e^{X} \star_{2} e^{Y}=\frac{q^{1 / 2}(X) J^{1 / 2}(Y)}{J^{1 / 2}\left(Z_{\mathrm{sym}}(X, Y)\right)} e^{Z_{\mathrm{sym}}(X, Y)} .
$$

Quand $s$ tend vers l'infini on trouve $A(X) e^{X} \star_{1} e^{Y}$. Il est alors opportun de considérer les contributions divisées par $A(X)$ pour faire apparaître le facteur $D_{\text {sym }}(X, Y)=$ $\frac{J^{1 / 2}(X) J^{1 / 2}(Y)}{J^{1 / 2}\left(Z_{\mathrm{sym}}(X, Y)\right)}$.

Pour $s$ quelconque, les contributions totales divisées par $A(X)$ sont de la forme

$$
D_{s}^{(1)}(X, Y) e^{Z_{\mathrm{sym}, s}^{(1)}(X, Y)},
$$

où $D_{s}^{(1)}(X, Y)$ est une fonction de densité et $Z_{\mathrm{sym}, s}^{(1)}(X, Y) \in \mathfrak{p}$ est une déformation de la fonction de Campbell-Hausdorff. La déformation est alors contrôlée par les concentrations en $e^{Y}$. Les graphes qui interviennent vont se factoriser comme dans [33, 34]. L'arête issue de la position $e^{Y}$ va sur le sommet d'un graphe représentant un élément dek. Notons $G_{s}^{(1)}(X, Y)$ les contributions de tous ces graphes; c'est une 1-forme en $s$.

Il vient alors les deux équations d'évolution suivante :

$$
\begin{gathered}
\mathrm{d}_{s} Z_{\mathrm{sym}, s}^{(1)}(X, Y)=\left[Y, G_{s}(X, Y)\right] \cdot \partial_{Y} Z_{\mathrm{sym}, s}^{(1)}(X, Y) \\
\mathrm{d}_{s} D_{s}^{(1)}(X, Y)=\left[Y, G_{s}(X, Y)\right] \cdot \partial_{Y} D_{s}^{(1)}(X, Y)+\operatorname{tr}_{\mathfrak{k}}\left(\partial_{Y} G_{s}(X, Y) \circ \operatorname{ad} Y\right) D_{s}^{(1)}(X, Y),
\end{gathered}
$$

avec condition limite pour $s=0$ :

$$
Z_{\mathrm{sym}, s=0}^{(1)}(X, Y)=Z_{\mathrm{sym}}(X, Y)
$$

et

$$
D_{s=0}^{(1)}(X, Y)=\frac{J^{1 / 2}(X) J^{1 / 2}(Y)}{J^{1 / 2}\left(Z_{\mathrm{sym}}(X, Y)\right)}=D_{\mathrm{sym}}(X, Y) .
$$

La condition limite pour $s=\infty$ correspond à $e^{X} \star_{1} e^{Y}$.

$4^{\text {e }}$ SÉRIE - TOME $41-2008-\mathrm{N}^{\mathrm{o}} 5$ 
Deuxième déformation. - Positionnons maintenant $e^{X}$ et $e^{Y}$ sur l'axe horizontal. Sans perte de généralité, on peut positionner $e^{Y}$ en 1 et $e^{X}$ en $\left.u \in\right] 0,1$ [. Lorsque $u$ tend vers 0 on trouve $\left(1 \star_{1} e^{X}\right) \star_{1} e^{Y}=B(X) e^{X} \star_{1} e^{Y}=e^{X} \star_{1} e^{Y}$. Quand $u$ tend vers 1 on trouve

$$
E(X, Y) e^{X+Y}
$$

le produit pour les paires symétriques.

Pour $u$ quelconque, les contributions totales sont de la forme

$$
D_{u}^{(2)}(X, Y) e^{Z_{\mathrm{sym}, u}^{(2)}(X, Y)}
$$

où $D_{u}^{(2)}(X, Y)$ désigne une fonction de densité et $Z_{\mathrm{sym}, u}^{(2)}(X, Y) \in \mathfrak{p}$ est une déformation de la fonction de Campbell-Hausdorff. La déformation est contrôlée par des champs $\mathfrak{k}$-adjoints agissant cette fois-ci sur les deux variables. Les concentrations sur $e^{X}$ et $e^{Y}$ vont définir des 1 -formes en $u$ représentant deux séries à valeurs dans $\mathfrak{k}, F_{u}(X, Y)$ (les contributions des graphes avec arête colorée par $\mathfrak{k}^{*}$ issue de $e^{X}$ ) et $G_{u}(X, Y)$ (les contributions des graphes avec arête colorée par $\mathfrak{k}^{*}$ issue de $e^{Y}$ ).

Il vient donc les équations suivantes :

$$
\begin{gathered}
\mathrm{d}_{u} Z_{\mathrm{sym}, u}^{(2)}(X, Y)=\left(\left[X, F_{u}(X, Y)\right] \cdot \partial_{X}+\left[Y, G_{u}(X, Y)\right] \cdot \partial_{Y}\right) Z_{\mathrm{sym}, u}^{(2)}(X, Y) \\
\mathrm{d}_{u} D_{u}^{(2)}(X, Y)=\left(\left[X, F_{u}(X, Y)\right] \cdot \partial_{X}+\left[Y, G_{u}(X, Y)\right] \cdot \partial_{Y}\right) D_{u}^{(2)}(X, Y)= \\
\operatorname{tr}_{\mathfrak{k}}\left(\partial_{X} F_{u}(X, Y) \circ \operatorname{ad} X+\partial_{Y} G_{u}(X, Y) \circ \operatorname{ad} X\right) D_{u}^{(2)}(X, Y) .
\end{gathered}
$$

Déformation de la formule de Campbell-Hausdorff pour les paires symétriques. - La jonction des deux déformations précédentes est alors continue et on en déduit le théorème suivant généralisant les résultats de [34].

ThÉORÈme 7. - Il existe une déformation régulière ${ }^{(39)} Z_{\mathrm{sym}, v}(X, Y)$ de la fonction de Campbell-Hausdorff pour les paires symétriques et une déformation régulière $D_{\mathrm{sym}, v}(X, Y)$ de la fonction de densité pour les paires symétriques telles que :

$$
\begin{aligned}
\mathrm{d}_{v} Z_{\text {sym }, v}(X, Y)= & \left(\left[X, F_{v}(X, Y)\right] \cdot \partial_{X}+\left[Y, G_{v}(X, Y)\right] \cdot \partial_{Y}\right) Z_{\text {sym }, v}(X, Y) \\
\mathrm{d}_{v} D_{\text {sym }, v}(X, Y)= & \left(\left[X, F_{v}(X, Y)\right] \cdot \partial_{X}+\left[Y, G_{v}(X, Y)\right] \cdot \partial_{Y}\right) D_{\text {sym }, v}(X, Y) \\
& +\operatorname{tr}_{\mathfrak{k}}\left(\partial_{X} F_{v}(X, Y) \circ \operatorname{ad} X+\partial_{Y} G_{v}(X, Y) \circ \operatorname{ad} X\right) D_{\text {sym }, v}(X, Y),
\end{aligned}
$$

où $F_{v}$ et $G_{v}$ sont des 1 -formes en $v$ et des séries à valeurs dans $\mathfrak{k}$ convergentes dans un voisinage de $(0,0)$. Les conditions limites sont pour $v=0$ :

$$
D_{\mathrm{sym}, v=0}(X, Y)=\frac{J^{1 / 2}(X) J^{1 / 2}(Y)}{J^{1 / 2}\left(Z_{\mathrm{sym}}(X, Y)\right)} \quad Z_{\mathrm{sym}, v=0}(X, Y)=Z_{\mathrm{sym}}(X, Y)
$$

et pour $v=\infty$ :

$$
D_{\mathrm{sym}, v=\infty}(X, Y)=E(X, Y) \quad Z_{\mathrm{sym}, v=\infty}(X, Y)=X+Y .
$$

(39) Continue en $s$ analytique par morceaux en $s$ et analytique en $X, Y$. 
Cette déformation, le long des axes, remplace la déformation dans le demi-plan complexe définie par Kontsevich et utilisée dans [33, 34]. En utilisant les techniques développées par A. Alekseev et E. Meinrenken dans [2] et expliquées de manière simplifiée dans [36], on montre facilement que la condition $E=1$ implique que la déformation par dilatation est aussi contrôlée par les champs $\mathfrak{k}$-adjoints (avec la condition de trace vérifiée). C'est le cas des paires symétriques résolubles ou les algèbres de Lie quadratiques considérées comme des paires symétriques.

On en déduit la proposition suivante.

Proposition 13. - Pour les algèbres de Lie quadratiques vues comme des paires symétriques, la déformation le long des axes fournit une solution à la conjecture de KashiwaraVergne. Plus généralement la conjecture $E=1$ pour les algèbres de Lie vues comme des paires symétriques implique la conjecture de Kashiwara-Vergne.

Considérons les solutions des équations différentielles dans le groupe $K$, d'algèbre de Lie $\mathfrak{k}$ avec condition initiale triviale pour $s=0$ :

$$
\mathrm{d}_{v} a_{v}(X, Y)=F_{v}(X, Y) a_{v}(X, Y) \quad \text { et } \quad \mathrm{d}_{v} b_{v}(X, Y)=G_{v}(X, Y) b_{v}(X, Y) .
$$

Comme dans [26, 27, 29], la fonction $v \mapsto D_{\mathrm{sym}, v}\left(a_{v}(X, Y) \cdot X, b_{v}(X, Y) \cdot X\right)$ vérifie une équation différentielle du type $y^{\prime}(v)=\mu(v) y(v)$ ce qui donne une formule pour $y(v)$.

Proposition 14. - On a l'expression de la fonction $E(X, Y)$ suivante

$$
\begin{aligned}
& E(X, Y)\left(\frac{J^{1 / 2}(X) J^{1 / 2}(Y)}{J^{1 / 2}\left(Z_{\mathrm{sym}}(X, Y)\right)}\right)^{-1}= \\
& \exp \left(\int_{0}^{\infty} \operatorname{tr}_{\mathfrak{k}}\left(\partial_{X} F_{v}\left(a_{v} \cdot X, b_{v} \cdot Y\right) \circ \operatorname{ad}\left(a_{v} \cdot X\right)+\partial_{Y} G_{v}\left(a_{v} \cdot X, b_{v} \cdot Y\right) \circ \operatorname{ad}\left(b_{v} \cdot Y\right)\right) \mathrm{d} v\right) .
\end{aligned}
$$

\section{Homomorphisme d'Harish-Chandra en termes de graphes}

On fixe une paire symétrique $(\mathfrak{g}, \sigma)$ et une décomposition d’Iwasawa (voir § 2.4)

$$
\mathfrak{g}=\mathfrak{k} \oplus \mathfrak{p}_{o} \oplus \mathfrak{n}_{+} .
$$

\subsection{Diagramme d'Harish-Chandra et espaces de réduction}

On place dans le diagramme de Cattaneo-Felder, pour la bi-quantification (§1.6), les sous-variétés co-isotropes $\mathfrak{k}^{\perp}$ en position horizontale et $\left(\mathfrak{k}_{o} \oplus \mathfrak{n}_{+}\right)^{\perp}$ en position verticale : c'est le diagramme d'Harish-Chandra.

On a la décomposition et les couleurs suivantes

$$
\mathfrak{g}=\underset{(-,+)}{\mathfrak{k} / \mathfrak{k}_{o}} \oplus \underset{(-,-)}{\mathfrak{k}_{o}} \oplus \underset{(+,+)}{\mathfrak{p}_{o}} \oplus \underset{(+,-)}{\stackrel{\mathfrak{n}_{+}}{ },}
$$

où on a noté $\mathfrak{k} / \mathfrak{k}_{o}$ un supplémentaire de $\mathfrak{k}_{o}$ dans $\mathfrak{k}$.

On fera attention que les graphes qui vont intervenir peuvent avoir une arête double si celle-ci est colorée par deux couleurs différentes.

$4^{\text {e }}$ SÉRIE - TOME $41-2008-\mathrm{N}^{\mathrm{o}} 5$ 
(i) L'algèbre de réduction, qui est placée verticalement, est tout simplement d'après la proposition $5(\S 2.4)$ :

$$
H_{\varepsilon}^{0}\left(\left(\mathfrak{k}_{o} \oplus \mathfrak{n}^{+}\right)^{\perp}\right)=\mathcal{C}_{\text {poly }}\left(\mathfrak{k}_{o}^{\perp}\right)^{\mathfrak{k}_{o}}[[\varepsilon]]=S\left(\mathfrak{p}_{o}\right)^{\mathfrak{k}_{o}}[[\varepsilon]] .
$$

(ii) L'algèbre de réduction placée à l'origine est aussi $S\left(\mathfrak{p}_{o}\right)^{\mathfrak{k}_{o}}[[\varepsilon]]$.

(iii) L'algèbre de réduction, qui est en position horizontale, correspond à l'espace de réduction $H_{\varepsilon}^{0}\left(\mathfrak{k}^{\perp}\right)$ où on a pris $\mathfrak{p}_{o} \oplus \mathfrak{n}_{+}$comme supplémentaire de $\mathfrak{k}$. On notera cet espace de réduction :

$$
H_{\varepsilon,\left(\mathfrak{p}_{o} \oplus \mathfrak{n}_{+}\right)}^{0}\left(\mathfrak{k}^{\perp}\right) .
$$

Il est isomorphe à l'espace de réduction standard $\left(S(\mathfrak{p})^{\mathfrak{k}}[[\varepsilon]], \underset{C F}{\star}\right)$ grâce au théorème $1(\S 1.5)$.

\subsection{Espace de réduction vertical}

Dans la partie verticale on trouve l'espace de réduction $S\left(\mathfrak{p}_{o}\right)^{\mathfrak{k}_{o}}$. Examinons le starproduit construit dans la proposition $5(\$ 2.4)$. Il est clairement donné par une fonction de type $\widehat{E}(X, Y)$, correspondant aux graphes de type Roue attachée à $e^{X}, e^{Y}$ pour $X, Y \in \mathfrak{p}_{o}$.

Proposition 15. - On a $\widehat{E}(X, Y)=E_{\mathfrak{g}_{o}}(X, Y)$, où $E_{\mathfrak{g}_{o}}(X, Y)$ est la fonction $E$ pour la petite paire symétrique $\left(\mathfrak{g}_{o}, \sigma\right)$. Le produit, dans la petite paire symétrique, est donc le produit de Rouvière standard.

Démonstration. - Examinons les contributions qui interviennent dans une roue attachée à des éléments de $\mathfrak{g}_{o}$. Les couleurs dans le cycle sont alors simples :

(i) soit le cycle dérive dans les directions de $\mathfrak{g}_{o}^{*}$, et on retrouve la fonction $E_{\mathfrak{g}_{o}}$ pour la petite paire symétrique,

(ii) soit le cycle dérive dans les directions de $\mathfrak{n}_{+}^{*}$ avec couleur $\rightarrow-\rightarrow$,

(iii) soit le cycle dérive dans les directions de $\left(\mathfrak{g} /\left(\mathfrak{g}_{o} \oplus \mathfrak{n}_{+}\right)\right)^{*}=\left(\mathfrak{k} / \mathfrak{k}_{o}\right)^{*}$ avec couleur $\longrightarrow$.

On a donc des contributions du genre

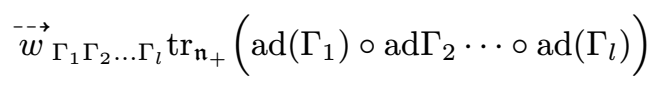

ou bien

$$
\begin{aligned}
\vec{w}_{\Gamma_{1} \Gamma_{2} \ldots \Gamma_{l}} \operatorname{tr}_{\mathfrak{g} /\left(\mathfrak{g}_{o} \oplus \mathfrak{n}_{+}\right)}\left(\operatorname{ad}\left(\Gamma_{1}\right) \circ \operatorname{ad} \Gamma_{2} \cdots \circ \operatorname{ad}\left(\Gamma_{l}\right)\right)= \\
\\
\vec{w}_{\Gamma_{1} \Gamma_{2} \ldots \Gamma_{l}} \operatorname{tr}_{\mathfrak{n}_{-}}\left(\operatorname{ad}\left(\Gamma_{1}\right) \circ \operatorname{ad} \Gamma_{2} \cdots \circ \operatorname{ad}\left(\Gamma_{l}\right)\right) .
\end{aligned}
$$

Remarquons alors que nous avons la symétrie $\stackrel{-\vec{w}}{w} \Gamma_{1} \Gamma_{2} \ldots \Gamma_{l}=(-1)^{l-1} \vec{w} \Gamma_{1} \Gamma_{l} \ldots \Gamma_{2}$.

Notons $V(X, Y)$ toutes les contributions de type (ii) ou (iii). Ici il se passe un phénomène remarquable. Comme la couleur est homogène dans le cycle, les dérivations se font soit toutes dans $\mathfrak{n}_{+}^{*}$ soit toutes dans $\left(\mathfrak{k} / \mathfrak{k}_{o}\right)^{*}$. L'argument de déformation à 4 points ([9] $\left.\S 8.5 .2\right)$, montre qu'il n'y a pas de trace dans l'équation de déformation. Par conséquent on peut faire intervenir la compensation dans $K_{o}$. On obtient au final :

$$
V(X, Y)^{2} D(X+Y)=D(a \cdot X) D(b \cdot Y),
$$


où $D(X)$ vaut $V(X,-X)$ et $a, b$ sont dans $K_{o}$. Comme $V$ est clairement invariante pour l'action de $K_{o}$, on obtient la factorisation souhaitée. De plus $D$ est symétrique et donc les roues qui interviennent sont de taille paire et attachées directement à l'axe réel. Or, pour $X \in \mathfrak{p}_{o}$ on $\operatorname{tr}_{\mathfrak{n}_{+}}(\operatorname{ad} X)^{2 n}=\operatorname{tr}_{\mathfrak{n}_{-}}(\operatorname{ad} X)^{2 n}$; des relations (5.2), (5.3), (5.4) on déduit que l'on a $D(X)=1$.

Remarque 9. - D'après [35], pour les petites paires symétriques, le produit de Rouvière correspond au produit standard de l'algèbre symétrique via la symétrisation. c'est-à-dire que la fonction $E_{\mathfrak{g}_{o}}$ est sans effet sur les éléments $\mathfrak{k}_{o}$-invariants.

\subsection{Espace de réduction horizontal}

Equations de réduction. - Les équations qui décrivent l'espace de réduction horizontal $H_{\varepsilon,\left(\mathfrak{p}_{o} \oplus \mathfrak{n}_{+}\right)}\left(\mathfrak{k}^{\perp}\right)$, sont données par des graphes colorés avec un seul point terrestre. Ce sont les graphes de la section 2.2. Les équations portent un degré (le nombre de sommets aériens) et les termes de degré pair sont nuls d'après le lemme 7 (\$2.2). L'opérateur $D_{1}$ correspond à l'action des champs ḱ-adjoints et l'opérateur $D_{3}$ contient un terme de type Bernoulli qui est non nul. Le dessin de gauche de (5.5) illustre un graphe pouvant intervenir dans $D_{5}$.

Ce sont des équations compliquées. En effet, les graphes qui apparaissent dans la détermination de l'espace de réduction sont de type Bernoulli ou de type Roue avec une sortie vers un Bernoulli. Les arêtes sortantes sont dans $\mathfrak{k}^{*}$, tandis que les arêtes qui arrivent sur l'axe réel sont colorées par $\mathfrak{p}_{o}^{*}$ ou $\mathfrak{n}_{+}^{*}$. Les arêtes intermédiaires peuvent prendre toutes les couleurs, ce qui ne permet pas des simplifications dans le calcul des coefficients ou de l'opérateur.

Explications. - L'espace de réduction pour ce choix de supplémentaire est, heuristiquement, l'écriture dans les coordonnées $\left(\mathfrak{n}_{+}, \mathfrak{p}_{o}\right)$ des éléments $\mathfrak{k}$-invariants de $U(\mathfrak{g}) / U(\mathfrak{g}) \cdot \mathfrak{k}$ via la décomposition

$$
U(\mathfrak{g}) / U(\mathfrak{g}) \cdot \mathfrak{k} \simeq U\left(\mathfrak{n}_{+}\right) \otimes U\left(\mathfrak{g}_{o}\right) / U\left(\mathfrak{g}_{o}\right) \cdot \mathfrak{k}_{o} \simeq S\left(\mathfrak{n}_{+}\right) \otimes S\left(\mathfrak{p}_{o}\right),
$$

où on a utilisé dans chaque facteur la symétrisation. On conçoit que l'écriture des éléments $\mathfrak{k}$-invariants ne soit pas simple!

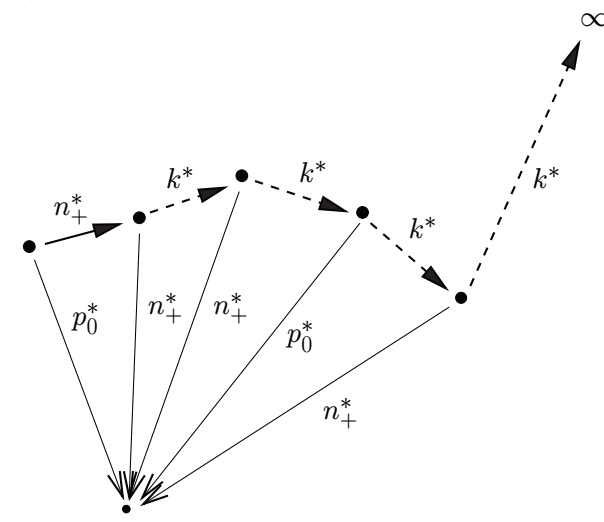

Graphe de $D_{5}$

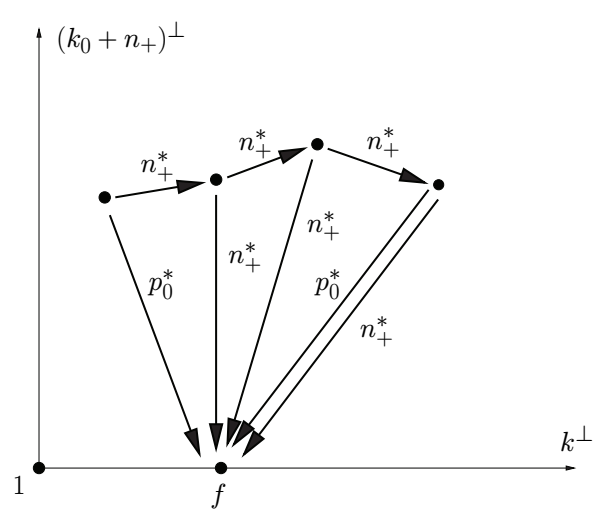

Transmutation horizontale

$4{ }^{\mathrm{e}}$ SÉRIE - TOME $41-2008-\mathrm{N}^{\circ} 5$ 
Description du produit $\star_{\mathfrak{p}_{o}+\mathfrak{n}_{+}} \cdot-$ Le produit est composé soit de graphes de type Lie, soit de type Roue.

i- Les graphes de type Lie ont forcément une racine dans $\mathfrak{n}_{+}$. En effet, comme les couleurs sur l'axe réel sont dans $\mathfrak{p}_{o}^{*}$ ou $\mathfrak{n}_{+}^{*}$, la racine est dans $\mathfrak{g}_{o}$ ou $\mathfrak{n}_{+}$. Si la racine est dans $\mathfrak{g}_{o}$, alors le coefficient est nul comme dans le cas des paires symétriques.

ii- Les graphes de type Roue se décomposent en deux sous-ensembles. Le raisonnement fait pour la description de l'espace de réduction verticale peut se recopier. On retrouve les contributions pour le petit espace symétrique, où les cycles sont colorés de manière uniforme par $\mathfrak{n}_{+}^{*}(=\rightarrow)$ ou par $\mathfrak{k}^{*} / \mathfrak{k}_{o}^{*}(=-\rightarrow)$. Pour calculer ces dernières contributions, il suffit de placer des points génériques $e^{X}$ et $e^{Y}$ sur l'axe réel avec $X, Y \in \mathfrak{p}_{o}$. L'argument à 4 points ([9] $\S$ 8.5.2) montre encore que l'on dispose d'une équation d'évolution sans trace, ce qui permet de compenser l'évolution par un élément de $K_{o}$. Pour les raisons des symétries on va trouver que ces contributions valent encore 1 .

\subsection{Entrelacement et projection}

Rappelons la décomposition et les couleurs dans le diagramme de Cattaneo-Felder associé à la décomposition d'Iwasawa :

$$
\mathfrak{g}=\underset{(-,+)}{\mathfrak{k} / \mathfrak{k}_{o}} \oplus \underset{(-,-)}{\mathfrak{k}_{o}} \oplus \underset{(+,+)}{\underset{\mathfrak{p}_{o}}{\ln _{(+,-)}} \oplus \underset{(+,}{\mathfrak{n}_{+}}} \cdot
$$

On définit l'opérateur de transmutation dans le diagramme $\left(\mathfrak{k}_{o} \oplus \mathfrak{n}_{+}\right)^{\perp}$ et $\mathfrak{k}^{\perp}$, c'est-à-dire que l'on va utiliser la structure de bi-module de $S\left(\mathfrak{p}_{o}\right)^{\mathfrak{k}_{o}}$ pour transmuter les éléments d'un côté vers l'autre. Pour $f \in H_{\varepsilon, \mathfrak{p}_{o}+\mathfrak{n}_{+}}\left(\mathfrak{k}^{\perp}\right)$ on va écrire

$$
\Gamma_{H C}(f) \underset{2}{\star} 1=1 \underset{1}{\star} f
$$

où $\Gamma_{H C}(f)$ est un polynôme invariant dans $S\left(\mathfrak{p}_{o}\right)^{\mathfrak{k}_{o}}$. L'opérateur $\Gamma_{H C}$ sera appelé opérateur de transmutation.

Lemme 16. - Les opérateurs qui interviennent dans la transmutation pour le diagramme d'Harish-Chandra sont des roues (a priori tentaculaires).

Démonstration. - Pour la partie horizontale, les dérivées se font dans les directions $\mathfrak{p}_{o}^{*}$ ou $\mathfrak{n}_{+}^{*}$. Donc pour les graphes de type Lie, la racine est dans $\mathfrak{n}_{+}$, ce qui va donner 0 pour l'opérateur quand on va le restreindre à $\left(\mathfrak{k}_{o} \oplus \mathfrak{n}_{+}\right)^{\perp} \cap \mathfrak{k}^{\perp}$. Donc toutes les arêtes qui arrivent sur l'axe réel sont dans $\mathfrak{p}_{o}^{*}$. Mais alors il y a une arête double de même couleur et le coefficient est nul.

Pour la partie verticale c'est plus simple. Comme l'espace de réduction est $S\left(\mathfrak{p}_{o}\right)^{\mathfrak{k}_{o}}$, seules les arêtes dans $\mathfrak{p}_{o}^{*}$ peuvent dériver. On a donc une arête double dans les graphes de type Bernoulli.

Notons ici $A_{H C}$ les contributions des roues horizontales et $B_{H C}$ les contributions des roues verticales sur les espaces de réductions respectifs.

Lemme 17. - Les roues intervenant dans $A_{H C}$ et $B_{H C}$ sont pures et ne dérivent que dans les directions de $\mathfrak{p}_{o}^{*}$. Les symboles correspondants sont donc des fonctions exponentielles sur $\mathfrak{p}_{o}$. 
Démonstration. - L'espace de réduction vertical est $S\left(\mathfrak{p}_{o}\right)^{\mathfrak{k}_{o}}$, donc les roues de $B_{H C}$ sont pures et ne dérivent que dans $\mathfrak{p}_{o}^{*}$.

Considérons une roue intervenant dans $A_{H C}$. Les extrémités dérivent dans les directions $\mathfrak{p}_{o}^{*}$ ou $\mathfrak{n}_{+}^{*}$. La couleur se propage dans l'arborescence, par conséquent les couleurs issues du cycle sont $\mathfrak{n}_{+}^{*}, \mathfrak{k}_{o}^{*}$ ou $\mathfrak{p}_{o}^{*}$. Supposons qu'à un sommet du cycle, il y ait une sortie (en dehors du cycle) colorée par $\mathfrak{n}_{+}^{*}$. En regardant la couleur de l'autre arête (celle qui reste dans le cycle), on se convainc que les couleurs dans le cycle sont :

(i) soit toutes dans $\mathfrak{n}_{+}^{*}$,

(ii) soit toutes dans $\left(\mathfrak{k} / \mathfrak{k}_{o}\right)^{*}$.

Alors les contributions dans le symbole, sont :

(i) $\operatorname{tr}_{\mathfrak{n}_{+}}\left(\operatorname{ad} P_{1} \cdots \operatorname{ad} N_{1} \cdots\right)=0$

ou bien

$$
\text { (ii) } \operatorname{tr}_{\mathfrak{g} /\left(\mathfrak{g}_{o} \oplus \mathfrak{n}_{+}\right)}\left(\operatorname{ad} P_{1} \cdots \operatorname{ad} N_{1} \cdots\right)=0 \text {. }
$$

On conclut que toutes les sorties du cycle sont dans $\mathfrak{p}_{o}^{*}$ et par conséquent les arêtes arrivant sur l'axe horizontal sont colorées par $\mathfrak{p}_{o}^{*}$; forcément les roues sont pures.

Proposition 16. - On a la relation $B_{H C}^{-1} A_{H C}=1$. L'opérateur de transmutation correspond simplement à la restriction $\grave{a}\left(\mathfrak{k} \oplus \mathfrak{n}_{+}\right)^{\perp}$.

Démonstration. - Les roues, dont le cycle est coloré par $\mathfrak{p}_{o}^{*}$ et $\mathfrak{k}_{o}^{*}$ (c'est-à-dire $(+,+)$ ou $(-,-))$, vérifient la même propriété que celle que nous avons déjà rencontrée dans la proposition 7 (§3.2). Il y a un nombre pair de sorties, avec alternance des couleurs $(+,+)$ et $(-,-)$, donc comme dans la Proposition 7, ces contributions s'annulent aussi bien dans $A_{H C}$ que dans $B_{H C}$, car les roues sont pures.

Il reste à considérer les roues, dont le cycle est de couleur uniforme en $(+,-)$ (dérivée en $\mathfrak{n}_{+}^{*}$ ) ou de couleur uniforme en $(-,+)$ (dérivée en $\mathfrak{k}^{*} / \mathfrak{k}_{o}^{*}$ ).

Notons $w_{\Gamma_{+-}}^{A}$ le coefficient associé à la roue pure de taille $n$ attachée sur l'axe horizontal et de couleur $(+,-)$. On introduit de même $w_{\Gamma_{-+}}^{A}, w_{\Gamma_{+-}}^{B}$ et $w_{\Gamma_{-+}}^{B}$.

Compte tenu des symétries (4.9) par rapport à la bissectrice principale on a :

$$
w_{\Gamma_{+-}}^{B}=(-1)^{n} w_{\Gamma_{-+}}^{A} \quad \text { et } \quad w_{\Gamma_{-+}}^{B}=(-1)^{n} w_{\Gamma_{+-}}^{A} .
$$

Par ailleurs du côté de l'opérateur, la dérivation ne se faisant qu'en la direction de $\mathfrak{p}_{o}^{*}$, le symbole correspondant peut s'écrire pour $X \in \mathfrak{p}_{o}$ :

$$
\begin{aligned}
\Gamma_{(+,-)}(X) & =\operatorname{tr}_{\mathfrak{n}_{+}}(\operatorname{ad} X)^{n} \\
\Gamma_{(-,+)}(X)=\operatorname{tr}_{\mathfrak{g} /\left(\mathfrak{g}_{o} \oplus \mathfrak{n}_{+}\right)}(\operatorname{ad} X)^{n} & =\operatorname{tr}_{\mathfrak{n}_{-}}(\operatorname{ad} X)^{n}=(-1)^{n} \operatorname{tr}_{\mathfrak{n}_{+}}(\operatorname{ad} X)^{n} .
\end{aligned}
$$

En effet on ${\operatorname{a~} t \mathfrak{n}_{+}}_{\mathfrak{n}_{+}} \operatorname{ad} X=-\operatorname{tr}_{\mathfrak{n}_{-}}$ad $X$ pour $X \in \mathfrak{p}_{o}$ car on a $\sigma\left(\mathfrak{n}_{+}\right)=\mathfrak{n}_{-}$et $\sigma(X)=-X$. Au total il y a compensation et on a les relations suivantes :

$$
w_{\Gamma_{+-}}^{B} \operatorname{tr}_{\mathfrak{n}_{+}}(\operatorname{ad} X)^{n}=w_{\Gamma_{-+}}^{A} \operatorname{tr}_{\mathfrak{n}_{-}}(\operatorname{ad} X)^{n}=w_{\Gamma_{-+}}^{A} \Gamma_{(+,-)}^{A}(X)
$$

et

$$
w_{\Gamma_{-+}}^{B} \Gamma_{(-,+)}(X)=w_{\Gamma_{+-}}^{A} \Gamma_{(+,-)}^{A}(X) .
$$

Ceci montre que l'on a $A_{H C}=B_{H C}$. Comme on a $\Gamma_{H C}(f)=B_{H C}^{-1} A_{H C}(f)$, on en déduit que $\Gamma_{H C}(f)$ est bien la restriction de $f$ à $\left(\mathfrak{k} \oplus \mathfrak{n}_{+}\right)^{\perp}$. 
Définition 6. - En théorie de Lie pour les paires symétriques, la projection sur le facteur $U\left(\mathfrak{g}_{o}\right) / U\left(\mathfrak{g}_{o}\right) \cdot \mathfrak{k}_{o}$ dans la décomposition

$$
U(\mathfrak{g}) / U(\mathfrak{g}) \cdot \mathfrak{k} \simeq U\left(\mathfrak{n}_{+}\right) \otimes U\left(\mathfrak{g}_{o}\right) / U\left(\mathfrak{g}_{o}\right) \cdot \mathfrak{k}_{o}
$$

s'appelle la projection d'Harish-Chandra. C'est un homomorphisme d'algèbres pour les invariants de

$$
(U(\mathfrak{g}) / U(\mathfrak{g}) \cdot \mathfrak{k})^{\mathfrak{k}} \quad \operatorname{sur} \quad\left(U\left(\mathfrak{g}_{o}\right) / U\left(\mathfrak{g}_{o}\right) \cdot \mathfrak{k}_{o}\right)^{\mathfrak{k}_{o}} .
$$

On peut maintenant énoncer un résultat similaire en termes de graphes de Kontsevich.

Proposition 17. - La projection d'Harish-Chandra correspond à l'opérateur de transmutation, c'est-à-dire la restriction à $\mathfrak{p}_{o}^{*}=\left(\mathfrak{k} \oplus \mathfrak{n}_{+}\right)^{\perp}$. Elle prend ses valeurs dans $S\left(\mathfrak{p}_{o}\right)^{\mathfrak{k}_{o}}$. C'est un homomorphisme d'algèbres de

$$
\left(H_{\varepsilon,\left(\mathfrak{p}_{o} \oplus \mathfrak{n}_{+}\right)}^{0}\left(\mathfrak{k}^{\perp}\right), \underset{\left(\mathfrak{p}_{o} \oplus \mathfrak{n}_{+}\right)}{\star}\right) \quad \operatorname{sur} \quad\left(S\left(\mathfrak{p}_{o}\right)^{\mathfrak{k}_{o}}[[\varepsilon]], \underset{\operatorname{Rouv}=\mathrm{CF}}{\star}\right) .
$$

Démonstration. - Le fait que la restriction à $\mathfrak{p}_{o}^{*}$ soit une fonction $\mathfrak{k}_{o}$-invariante est clair, car toutes les opérations que nous avons effectuées peuvent être choisies $\mathfrak{k}_{o}$-équivariantes. En effet le choix du supplémentaire $\mathfrak{k} / \mathfrak{k}_{o}$ peut être facilement choisi $\mathfrak{k}_{o}$-invariant, il suffit de prendre $\left(\mathfrak{n}_{-} \oplus \mathfrak{n}_{+}\right) \cap \mathfrak{k}$. Les équations sont clairement indépendantes des choix de la base (une fois choisies les décompositions), car cela correspond à des transformations linéaires qui préservent les couleurs des graphes. Maintenant $\mathfrak{k}_{o}$ préserve la décomposition. Donc l'espace de réduction est $K_{o}$-invariant.

Commentaire. - Il est bien connu que l'homomorphisme d'Harish-Chandra est quelque chose de très compliqué. Ici il correspond à une simple restriction. Il faut comprendre que la difficulté est cachée dans la description de l'algèbre de réduction. En quelque sorte il

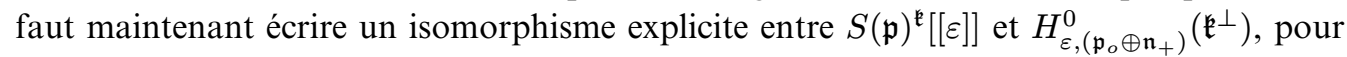
décrypter toute la difficulté de l'homomorphisme d'Harish-Chandra. C'est l'objet de la section suivante.

\subsection{L'homomorphisme d'Harish-Chandra en termes de graphes}

Le théorème $1(\S 1.5)$ montre qu'il existe un isomorphisme entre les deux espaces de réduction correspondant aux décompositions de Cartan et d'Iwasawa. On disposera alors d'une formule pour l'homomorphisme d'Harish-Chandra en termes de graphes. Il existe un isomorphisme d'algèbres de $\left(S(\mathfrak{p})^{\mathfrak{k}}, \underset{\mathrm{CF}}{\star}\right)$ dans $\left(S\left(\mathfrak{p}_{o}\right)^{\mathfrak{k}_{o}}, \underset{\mathrm{CF}}{\star}\right)$; c'est l'homomorphisme d'HarishChandra.

Calcul de l'élément de jauge. - On reprend ici les notations de $\S 1.5$. Le champ $v$ s'écrit $v=-V_{a} \partial_{P_{a}^{*}}$, où $\left(P_{a}^{*}\right)_{a}$ désigne une base de $\mathfrak{k}^{\perp}=\mathfrak{p}^{*}$ et $V_{a} \in \mathfrak{k}$.

L'opérateur qui réalise l'entrelacement est calculé de manière récursive. Plus précisément on doit intégrer la composante de degré (total) 0 du champ $\left[D U_{\pi_{t}}(v), \bullet\right]_{G H}$. C'est en effet cet élément qui conjugue les différentielles et réalise l'isomorphisme en cohomologie pour les star-produits, comme on l'a vu dans le théorème 1. 
Lemme 18. - L'élément du groupe de jauge, qui entrelace les star-produits pour deux choix de supplémentaires, vivra dans le groupe associé à l'algèbre de Lie engendrée par les coefficients de Taylor du champ $\left[\left(D U_{\widehat{\pi_{t}}}(\widehat{v})\right)_{0}, \bullet\right]_{G H}$.

Démonstration. - L'équation suivante sur les composantes de degré 0 (dans le complexe de Hochschild) contrôle la déformation des star-produits :

$$
\frac{\partial\left(\mu_{t}\right)_{0}}{\partial t}=\left[\left(D U_{\widehat{\pi_{t}}}(\widehat{v})\right)_{0},\left(\mu_{t}\right)_{0}\right]
$$

Pour simplifier, notons $A(t)=\left[D U_{\widehat{\pi_{t}}}(\widehat{v})_{0}, \bullet\right]$ l'opérateur linéaire d'ordre 0 (total et de Hochschild) et $y(t)=\left(\mu_{t}\right)_{0}$.

On doit donc résoudre une équation différentielle linéaire de la forme $y^{\prime}(t)=A(t) y(t)$. On a, comme série formelle en $t: y(t)=y(0)+\sum_{n \geq 1} \frac{t^{n}}{n !} y_{n}$. L'équation différentielle donne $y_{1}=A(0) y_{0}$. Posons $y^{(1)}(t)=e^{-t A(t)} y(t)$. Alors $y^{(\overline{1})}(t)=y_{0}\left(\bmod t^{2}\right)$ et vérifie l'équation

$$
\frac{\mathrm{d}}{\mathrm{d} t} y^{(1)}(t)=t\left(\frac{e^{-\operatorname{ad}(t A(t))}-1}{\operatorname{ad}(t A(t))} A^{\prime}(t)\right) y^{(1)}(t) .
$$

On pose $A_{1}(t)=\left(\frac{e^{-\operatorname{ad}(t A(t))}-1}{\operatorname{ad}(t A(t))} A^{\prime}(t)\right)$, qui est dans l'algèbre de Lie engendrée par les coefficients de Taylor de $A(t)$. Il vient l'équation différentielle

$$
\frac{\mathrm{d} y^{(1)}(t)}{\mathrm{d} t}=t A_{1}(t) y^{(1)}(t)
$$

On pose ensuite $y^{(2)}(t)=e^{-\frac{t^{2}}{2} A_{1}(t)} y^{(1)}(t)=e^{-\frac{t^{2}}{2} A_{1}(t)} e^{-t A(t)} y(t)$. Alors on a $y^{(2)}(t)=y_{0}\left(\bmod t^{3}\right)$ et on a, via la formule de Campbell-Hausdorff,

$$
y^{(2)}(t)=e^{\left(-\frac{t^{2}}{2} A_{1}(t)-t A(t)+\frac{1}{2}\left[\frac{t^{2}}{2} A_{1}(t), t A(t)\right] \cdots\right)} y(t) .
$$

Par récurrence on trouve que $y(t)$ est l'image (comme série formelle en $t$ ) de $y(0)$ par l'action d'un élément du groupe formel associé à l'algèbre de Lie des coefficients de Taylor de $A(t)$. On a de plus comme série formelle en $t$

$$
y(t)=e^{\Omega(t)} y(0)=e^{t A(t)} e^{\frac{t^{2}}{2} A_{2}(t)} \cdots y(0)
$$

La résolution sous cette forme est connue en analyse numérique [15] et se trouve explicitement dans [22] ${ }^{(40)}$.

Étude de l'opérateur $\left(D U_{\pi}(v)\right)_{0}$. - On a $\pi_{t=0}=\pi$. Dans le cas des paires symétriques, on montre maintenant que l'opérateur $D U_{\pi_{t}}(v)_{0}$ est nul pour $t=0$.

Lemme 19. - Les graphes qui interviennent dans la composante de Hochschild de degré 0 de $D U_{\pi}(v)$ que l'on note $\left(D U_{\pi}(v)\right)_{0}$ sont de trois types : des graphes de type Bernoulli fermés par $v$ (5.8) (dessin de gauche), des graphes de type Roue attachée à un Bernoulli lequel s'attache à $v$ (5.8) (dessin du milieu) ou des graphes de type Roue pure attachée à $v$ (5.8) (dessin de droite). L'opérateur $D U_{\pi}(v)_{0}$ est nul mais $\left(D U_{\pi}(v)\right)_{1}$ n'est pas nul.

(40) Référence que nous a indiquée D. Manchon et qui réfère à Zassenhaus.

$4^{\text {e }}$ SÉRIE - TOME $41-2008-\mathrm{N}^{\mathrm{o}} 5$ 
Démonstration. - Il suffit de regarder ce qui se passe lorsqu'on applique l'opérateur sur des fonctions, car les dérivées sortant de l'axe réel n'interviennent pas, sinon il sortirait une arête d'un sommet aérien ce qui est exclu (on aura alors $n \geq 2$ ).

L'opérateur n'agit donc que sur les coefficients de la fonction $f$, placée au point terrestre. Le sommet où on a placé le champ de vecteurs $v$ doit être dérivé dans la direction de $\mathfrak{k}^{*}$ (car les coefficients du champ $v$ sont dans $\mathfrak{k}$ ). Cette arête provient :

- soit d'un graphe de type Bernoulli lui-même attaché ou non à une roue,

- soit d'une roue pure.

Dans le premier cas, l'arête issue de $v$, peut soit dériver la racine du graphe de Bernoulli ${ }^{(41)}(5.8)$ (dessin de gauche), soit dériver le sommet terrestre si le graphe de Bernoulli est attaché à une roue (5.8) (dessin du milieu).

Dans le second cas, l'arête issue de $v$ doit dériver le sommet sur l'axe réel (5.8) (dessin de droite).

Il est facile de se convaincre, en examinant tous les cas, qu'il y a forcément un nombre $n$ impair de sommets attachés au bi-vecteur $\pi$. Par conséquent, la symétrie par rapport à l'axe vertical fait apparaître un signe $(-1)^{n}$ dans son coefficient, ce qui montre que les coefficients de ces graphes sont nuls. On a $\left(D U_{\pi}(v)\right)_{0}=0$.

Le terme de degré 1 (pour le complexe de Hochschild) de l'opérateur $D U_{\pi}(v)=0$ n'est pas nul car le graphe avec un seul sommet aérien et deux points terrestres intervient de manière non triviale (le coefficient vaut $\frac{1}{2}$ ).

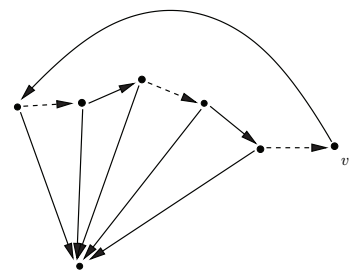

Bernoulli fermé par v Roue - Bernoulli attaché à v

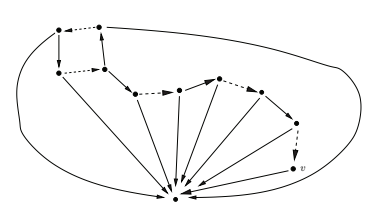

Coefficients de Taylor et invariance par le groupe de Weyl généralisé. - La description dia-

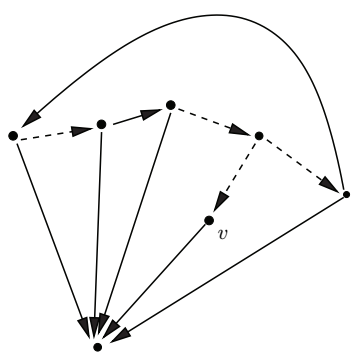

Roue pure sur $v$ grammatique de l'homomorphisme d'Harish-Chandra ne fait pas intervenir explicitement le groupe de Weyl généralisé.

Il faut montrer que nos constructions sont indépendantes du choix de $\mathfrak{n}_{+}$(choix d'un système de racines positives) et cela revient essentiellement à montrer que ces constructions sont invariantes lorsqu'on change $v$ en $-v$ (voir [32]).

Proposition 18. - Les coefficients de Taylor du champ $\left(D U_{\pi_{t}}(v)\right)_{0}$ sont invariants par le changement de champ $v \mapsto-v$. L'homomorphisme d'Harish-Chandra ne dépend donc pas $d u$ choix de la chambre de Weyl.

(41) Ce sommet doit être dérivé car il est dans k. 
Démonstration. - On fait un inventaire des graphes pour les coefficients de Taylor et on calcule le nombre de fois que $v$ apparait.

D'après les formules du $\S 1.5$, les bi-vecteurs $\pi$ et $[v,[v, \pi]]$ se comportent de la même manière vis-à-vis des couleurs, tandis que le bi-vecteur $[v, \pi]$ se comporte de manière opposée.

Les graphes qui apparaissent dans le calcul des coefficients de Taylor de $\left(D U_{\pi_{t}}(v)\right)_{0}$ sont les graphes décrits dans le lemme 19 auquel il faut ajouter le graphe de type Bernoulli non fermé (l'arête issue de $v$ dérive le sommet terrestre et la racine du Bernoulli est dans $\mathfrak{p}$ ). Les sommets, en dehors de $v$, sont attachés aux bi-vecteurs $\pi,[v, \pi]$ ou $[v,[v, \pi]]$.

Chaque sommet attaché à $\pi$ ou $[v,[v, \pi]]$ (on note $p$ leur nombre) engendre un changement de couleur dans le cycle de la roue ou le brin de Bernoulli, tandis que les sommets attachés à $[v, \pi]$ (on note $q$ leur nombre) ne changent pas la couleur. On doit avoir $p+q+1$ impair pour que le coefficient ne soit pas nul. Le nombre de fois que $v$ apparaît est congru à $q+1$.

1. Examinons le cas des graphes de type Bernoulli (fermé ou non) :

Le nombre $p$ doit être impair. En effet si le graphe est de type Bernoulli fermé alors l'arête issue de $v$ dérive la racine dans la couleur $\mathfrak{p}^{*}$. Si le graphe est de type Bernoulli non fermé les arêtes issues de la racine sont colorées par $\mathfrak{p}^{*}$. Dans tous les cas $q+1$ est pair. Le nombre de fois que $v$ apparaît est donc pair.

2. Examinons le cas des graphes de type Roue attachée à un Bernoulli :

On note $p_{1}$ (resp. $q_{1}$ ) le nombre de sommets, dans la roue, attachés à $\pi$ ou $[v,[v, \pi]]$ (resp. $[v, \pi]$ ). On note $p_{2}$ (resp. $q_{2}$ ) le nombre de sommets, dans le brin de Bernoulli, attachés à $\pi$ ou $[v,[v, \pi]]$ (resp. $[v, \pi]$ ). Le nombre de changements de couleur dans le cycle est pair. On en déduit facilement que $p_{1}+p_{2}$ est impair. Donc $q_{1}+q_{2}+1$ est pair.

Rappelons que pour les paires symétriques, la décomposition d’Iwasawa généralisée provient de l'action d'un tore $\mathfrak{s}_{f}$ généralisant les sous-espaces de Cartan, c'est le sous-espace de Cartan-Duflo (voir §2.4). Le groupe fini qui remplace le groupe de Weyl est le groupe quotient $N_{K}\left(\mathfrak{s}_{f}\right) / Z_{K}\left(\mathfrak{s}_{f}\right)$ du normalisateur sur le centralisateur. Remplacer $v$ par $-v$ revient dans notre situation à remplacer $\mathfrak{n}_{+}$par $\mathfrak{n}_{-}$. On en déduit en faisant intervenir des projections d'Harish-Chandra partielles que notre construction est indépendante du choix du système de racines positives.

On en déduit le corollaire suivant :

Corollaire 8. - L'homomorphisme d'Harish-Chandra est indépendant du choix du $\mathfrak{n}_{+}$. Il est invariant par le groupe de Weyl généralisé.

\section{Construction de caractères}

On considère $(\mathfrak{g}, \sigma)$ une paire symétrique et $\mathfrak{g}=\mathfrak{k} \oplus \mathfrak{p}$ sa décomposition de Cartan.

Dans cette section, on construit de manière systématique des caractères pour l'algèbre des opérateurs différentiels invariants en utilisant la bi-quantification ( $c f . \S 1.6)$ et des polarisations. On renvoie au $\S 2.5$ pour la notion de polarisation. 


\subsection{Construction de caractère pour des algèbres d'opérateurs différentiels invariants}

Principe de construction. - On applique le principe de bi-quantification aux sous-variétés co-isotropes $f+\mathfrak{b}^{\perp}$ (en position verticale) et $\mathfrak{k}^{\perp}$ (en position horizontale) avec $f \in \mathfrak{k}^{\perp}$ et $\mathfrak{b}$ une polarisation en $f$.

L'algèbre de réduction horizontale dépend d'un choix de supplémentaire de $\mathfrak{k}$ dans $\mathfrak{g}$, construit de manière compatible avec $\mathfrak{b}$; c'est-à-dire qu'on doit choisir un supplémentaire de $\mathfrak{b} \cap \mathfrak{k}$ dans $\mathfrak{b}$ (que l'on pourrait noter abusivement $\mathfrak{b} /(\mathfrak{b} \cap \mathfrak{k})$ ), puis un supplémentaire de $\mathfrak{k}+\mathfrak{b}$ dans $\mathfrak{g}$ (que l'on pourrait noter $\mathfrak{g} /(\mathfrak{k}+\mathfrak{b})$ ). On notera $\left(H_{\varepsilon, \mathfrak{b}}^{0}\left(\mathfrak{k}^{\perp}\right), \underset{\mathrm{CF}}{\star}\right)$ cette algèbre.

Pour $P \in H_{\varepsilon, \mathfrak{b}}^{0}\left(\mathfrak{k}^{\perp}\right)$ on calcule alors $1 \underset{1}{\star} P$. C'est une fonction polynomiale $K \cap B$ invariante sur $f+(\mathfrak{k}+\mathfrak{b})^{\perp}$, où $K$ et $B$ sont des groupes connexes d'algèbres de Lie $\mathfrak{k}$ et $\mathfrak{b}$. Or $(K \cap B) \cdot f$ est ouvert dans $f+(\mathfrak{k}+\mathfrak{b})^{\perp}$, donc cette fonction polynomiale est constante. L'application $P \mapsto 1 \star P \in \mathbb{R}[[\varepsilon]]$ est donc un caractère de l'algèbre associative $H_{\varepsilon, \mathfrak{b}}^{0}\left(\mathfrak{k}^{\perp}\right)$.

Proposition 19. - Le diagramme de Cattaneo-Felder appliqué au cas $f+\mathfrak{b}^{\perp}$ et $\mathfrak{k}^{\perp}$ avec $f \in \mathfrak{k}^{\perp}$ et $\mathfrak{b}$, une polarisation en $f$, fournit un caractère de l'algèbre de réduction $H_{\varepsilon, \mathfrak{b}}^{0}\left(\mathfrak{k}^{\perp}\right)$.

Grâce au théorème $1(\S 1.5)$, il existe un isomorphisme d'algèbres entre $H_{\varepsilon}^{0}\left(\mathfrak{k}^{\perp}\right)$ et $H_{\varepsilon, \mathfrak{b}}^{0}\left(\mathfrak{k}^{\perp}\right)$. En composant par cet isomorphisme, on construit donc un caractère pour l'algèbre de réduction qui dépend a priori du choix de la polarisation $\mathfrak{b}$ en $f^{(42)}$.

Remarque 10 (importante). - Dans le cas d'une sous-algèbre $\mathfrak{h}$ quelconque, cette méthode fournira un caractère de l'algèbre de réduction $H_{\varepsilon}^{0}\left(\mathfrak{h}^{\perp}\right)$, pour peu que l'on ait $(H \cap B) \cdot f$ ouvert dans $f+(\mathfrak{h}+\mathfrak{b})^{\perp}$. Sous cette hypothèse, $H \cdot f$ est nécessairement lagrangien dans $G \cdot f$ et on retrouve une condition évoquée dans [13], pour la commutativité de l'algèbre des opérateurs différentiels invariants sur $G / H$.

\subsection{Indépendance par rapport aux choix de la polarisation}

La méthode consiste à considérer une variation à 8 couleurs des graphes. On retrouve aux bords les graphes à 4 couleurs, ce qui permet l'interpolation entre les deux situations.

Construction de la forme à 8 couleurs

Proposition 20. - Il existe une 1-forme à 8 couleurs qui interpole les 1-formes à 4 couleurs.

Démonstration. - Le reste de ce paragraphe est consacré à la construction de cette 1-forme à 8 couleurs.

Considérons la demi-bande

$$
\sqsubset:=\left\{\mathbf{x}=(x, y) \in \mathbb{R}^{2}: x \geq 0,-\frac{\pi}{2} \leq y \leq \frac{\pi}{2}\right\} .
$$

(42) On peut se demander si ce caractère est indépendant du choix du supplémentaire de $\mathfrak{k}+\mathfrak{b}$ dans $\mathfrak{g}$. 
Notons $\sqsubseteq_{i}, i=1,2,3$, les trois composantes de son bord :

$$
\begin{aligned}
& \sqsubseteq_{1}:=\left\{\mathbf{x} \in \sqsubset: y=-\frac{\pi}{2}\right\}, \\
& \sqsubseteq_{2}:=\{\mathbf{x} \in \sqsubset: x=0\}, \\
& \sqsubseteq_{3}:=\left\{\mathbf{x} \in \sqsubset: y=\frac{\pi}{2}\right\} .
\end{aligned}
$$

Sur la compactification de l'espace de configurations $C_{2}(\sqsubset)$, on considère l'involution

$$
\begin{aligned}
p: \quad C_{2}(\sqsubset) & \rightarrow C_{2}(\sqsubset) \\
\left(\mathbf{x}_{1}, \mathbf{x}_{2}\right) & \mapsto\left(\mathbf{x}_{2}, \mathbf{x}_{1}\right) .
\end{aligned}
$$

On veut définir huit 1 -formes fermées $\theta_{j_{1} j_{2} j_{3}}, j_{i} \in\{1,2\}$, sur $C_{2}(\sqsubset)$ ayant les propriétés suivantes :

1. $\theta_{j_{1} j_{2} j_{3}}$ s'annulent quand $\mathbf{x}_{j_{i}}$ approche de $\sqsubseteq_{i}, i=1,2,3$;

2. sur la composante de bord correspondant au rapprochement de $\mathbf{x}_{1}$ et $\mathbf{x}_{2}$ tout en restant à l'intérieur de $\sqsubset, \theta_{j_{1} j_{2} j_{3}}$ s'identifie à la forme volume normalisée et invariante sur $\mathbb{S}^{1}$;

3. pour $j_{i}=1\left(j_{i}=2\right), i=1,2,3$, sur la composante de bord correspondant au rapprochement de $\mathbf{x}_{1}$ et $\mathbf{x}_{2}$ près de l'intérieur de ${ }_{i}, \theta_{j_{1} j_{2} j_{3}}\left(p^{*} \theta_{j_{1} j_{2} j_{3}}\right)$ est la 1-forme de Kontsevich;

4. sur le bord correspondant au rapprochement de $\mathbf{x}_{1}$ et $\mathbf{x}_{2}$ près d'un coin, $\theta_{j_{1} j_{2} j_{3}}$ est la 1-forme à 4 couleurs de Cattaneo-Felder (\$1.6).

Cas $j_{1} \neq j_{3}$. - Soit $\theta$ la forme tautologique sur $C_{2}\left(\mathbb{R}^{2}\right)$ correspondant à la métrique euclidienne; c'est-à-dire, $\theta:=\phi^{*} \omega$, où $\omega$ est la 1 -forme volume normalisée et invariante sur $\mathbb{S}^{1}$ et

$$
\begin{aligned}
\phi: C_{2}\left(\mathbb{R}^{2}\right) & \rightarrow \mathbb{S}^{1} \\
\left(\mathbf{x}_{1}, \mathbf{x}_{2}\right) & \mapsto \frac{\mathbf{x}_{2}-\mathbf{x}_{1}}{\left\|\mathbf{x}_{2}-\mathbf{x}_{1}\right\|} .
\end{aligned}
$$

Soit $\tau_{i}: \mathbb{R}^{2} \rightarrow \mathbb{R}^{2}(i=1,2,3)$ l'involution correspondant à la réflexion par rapport aux droites supportant $\sqsubseteq_{i}$; c'est-à-dire :

$$
\begin{aligned}
& \tau_{1}(x, y)=(x,-\pi-y), \\
& \tau_{2}(x, y)=(-x, y), \\
& \tau_{3}(x, y)=(x, \pi-y) .
\end{aligned}
$$

Remarquons que l'on a $\tau_{1} \circ \tau_{2}=\tau_{2} \circ \tau_{1}$ et $\tau_{3} \circ \tau_{2}=\tau_{2} \circ \tau_{3}$.

Soit $C_{2}\left(\mathbb{R}^{2}\right)^{\prime}$ le sous-ensemble de $C_{2}\left(\mathbb{R}^{2}\right)$ défini par $\mathbf{x}_{1} \neq \tau_{i}\left(\mathbf{x}_{2}\right)$ pour $i=1,2,3$. Observons que l'on a $C_{2}(\sqsubset) \subset C_{2}\left(\mathbb{R}^{2}\right)^{\prime}$. Écrivons $\tau_{i j}(i=1,2,3 ; j=1,2)$ pour l'action de $\tau_{i}$ sur la composante $j$ de $C_{2}\left(\mathbb{R}^{2}\right)^{\prime}$; c'est-à-dire :

$$
\begin{aligned}
\tau_{i 1}: & C_{2}\left(\mathbb{R}^{2}\right)^{\prime} \\
\left(\mathbf{x}_{1}, \mathbf{x}_{2}\right) & \left.\mapsto\left(\tau_{2}\left(\mathbb{R}^{2}\right)^{\prime}\right), \mathbf{x}_{2}\right) \\
\tau_{i 2}: & C_{2}\left(\mathbb{R}^{2}\right)^{\prime} \rightarrow C_{2}\left(\mathbb{R}^{2}\right)^{\prime} \\
\left(\mathbf{x}_{1}, \mathbf{x}_{2}\right) & \mapsto\left(\mathbf{x}_{1}, \tau_{i}\left(\mathbf{x}_{2}\right)\right) .
\end{aligned}
$$


Remarquons que l'on a

$$
\begin{array}{ll}
\tau_{1 j_{1}} \circ \tau_{2 j_{2}}=\tau_{2 j_{2}} \circ \tau_{1 j_{1}} & \forall j_{1}, j_{2}, \\
\tau_{3 j_{3}} \circ \tau_{2 j_{2}}=\tau_{2 j_{2}} \circ \tau_{3 j_{3}} & \forall j_{1}, j_{3}, \\
\tau_{1 j_{1}} \circ \tau_{3 j_{3}}=\tau_{3 j_{3}} \circ \tau_{1 j_{1}} & \forall j_{1} \neq j_{3} .
\end{array}
$$

Alors, si on fixe $j_{1}, j_{2}, j_{3}$ avec $j_{1} \neq j_{3}$, le groupe engendré par $\tau_{1 j_{1}}, \tau_{2 j_{2}}, \tau_{3 j_{3}}$ est abélien et fini (d'ordre 8). Enfin, on définit

$$
\theta_{j_{1} j_{2} j_{3}}=\iota_{C_{2}(\sqsubset)}^{*}\left(\sum_{a, b, c=0,1}(-1)^{a+b+c}\left(\tau_{1 j_{1}}^{*}\right)^{a}\left(\tau_{2 j_{2}}^{*}\right)^{b}\left(\tau_{3 j_{3}}^{*}\right)^{c} \iota_{C_{2}\left(\mathbb{R}^{2}\right)^{\prime}}^{*} \theta\right),
$$

où $\iota_{\bullet}^{*}$ est la restriction à $\bullet$. Il n'est pas difficile de vérifier que les formes $\theta_{j_{1} j_{2} j_{3}}, j_{1} \neq j_{3}$, satisfont aux conditions souhaitées.

Cas $j_{1}=j_{3} .-$ La construction précédente ne s'applique pas dans le cas $j_{1}=j_{3}$ car $\tau_{1}$ et $\tau_{3}$ ne commutent pas. Nous avons besoin d'une autre construction.

Soit $S$ la bande $\left\{\mathbf{x}=(x, y) \in \mathbb{R}^{2}:-\frac{\pi}{2} \leq y \leq \frac{\pi}{2}\right\}$. Sur (l'intérieur de) $S$ on considère la métrique

$$
\mathrm{d} s^{2}=\frac{\mathrm{d} x^{2}+\mathrm{d} y^{2}}{\cos ^{2} y}
$$

qui approche la métrique de Poincaré sur chaque composante de bord. Les géodésiques sont,

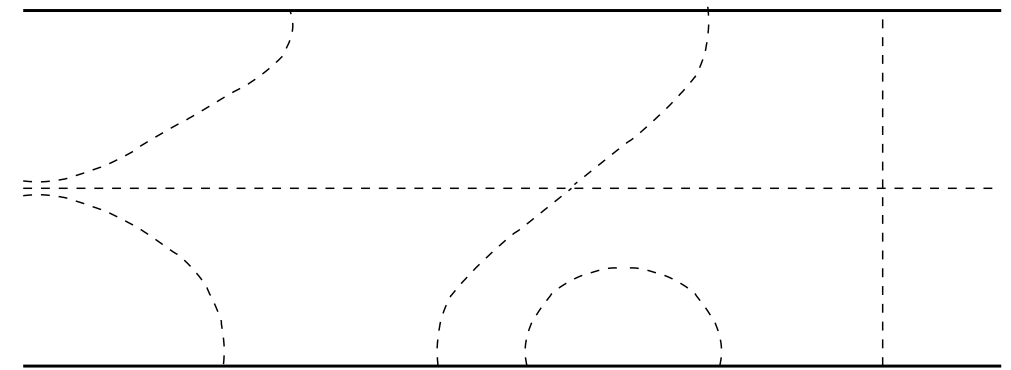

Figure 6. Géodésiques dans la bande

soit des lignes verticales, soit des courbes de la forme $\sin y=A \mathrm{e}^{x}+B \mathrm{e}^{-x}$, où $A$ et $B$ sont des paramètres.

Remarquons qu'il existe une et une seule géodésique passant par deux points distincts de $S$. On définit la fonction d'angle $\phi\left(\mathbf{x}_{1}, \mathbf{x}_{2}\right)$ comme l'angle entre la géodésique verticale passant par $\mathbf{x}_{1}$ et la géodésique joignant $\mathbf{x}_{1}$ à $\mathbf{x}_{2}$. On définit ensuite $\vartheta$ comme $\mathrm{d} \phi /(2 \pi)$. Alors $\vartheta$ est une 1-forme fermée sur $C_{2}(S)$ satisfaisant aux propriétés suivantes :

1. $\vartheta$ s'annule quand $\mathbf{x}_{1}$ s'approche du bord de $S$;

2. sur la composante de bord, correspondant au rapprochement de $\mathbf{x}_{1}$ et $\mathbf{x}_{2}$ tout en restant dans l'intérieur de $S, \vartheta$ s'identifie à la forme volume normalisée et invariante sur $\mathbb{S}^{1}$ 
3. sur la composante de bord correspondant au rapprochement de $\mathbf{x}_{1}$ et $\mathbf{x}_{2}$ près du bord de $S, \vartheta$ est la 1-forme de Kontsevich.

Soit $\sigma: S \rightarrow S$ l'involution correspondant à la réflexion par rapport à $x=0$ : c'est-à-dire, $\sigma(x, y)=(-x, y)$. Soit $C_{2}(S)^{\prime}$ le sous-ensemble de $C_{2}(S)$ défini par $\mathbf{x}_{1} \neq \sigma\left(\mathbf{x}_{2}\right)$. Remarquons que l'on a $C_{2}(\sqsubset) \subset C_{2}(S)^{\prime}$. On écrit $\sigma_{j}(j=1,2)$ pour l'action de $\sigma$ sur la composante $j$ de $C_{2}(S)^{\prime}$; c'est-à-dire :

$$
\begin{aligned}
\sigma_{1}: & C_{2}(S)^{\prime} \rightarrow C_{2}(S)^{\prime} \\
\left(\mathbf{x}_{1}, \mathbf{x}_{2}\right) & \mapsto\left(\sigma\left(\mathbf{x}_{1}\right), \mathbf{x}_{2}\right) \\
\sigma_{2}: & C_{2}(S)^{\prime} \rightarrow C_{2}(S)^{\prime} \\
\left(\mathbf{x}_{1}, \mathbf{x}_{2}\right) & \mapsto\left(\mathbf{x}_{1}, \sigma\left(\mathbf{x}_{2}\right)\right)
\end{aligned}
$$

Enfin, on définit

$$
\theta_{1 j 1}=\iota_{C_{2}(\sqsubset)}^{*}\left(\sum_{a=0,1}(-1)^{a}\left(\sigma_{j}^{*}\right)^{a} \iota_{C_{2}(S)^{\prime}}^{*} \vartheta\right),
$$

et

$$
\theta_{2 j 2}=p^{*} \theta_{1,3-j, 1} .
$$

Il n'est pas difficile de vérifier que les 1 -formes $\theta_{j_{1} j_{2} j_{3}}, j_{1}=j_{3}$, satisfont aux conditions désirées.

Polarisations en position d'intersection normale. - On utilise la forme à 8 couleurs dans le cas de 3 sous-algèbres $\mathfrak{k}, \mathfrak{b}_{1}, \mathfrak{b}_{2}$ en position d'intersections normales. c'est-à-dire qu'il faut que l'on ait (on peut intervertir les rôles de $\mathfrak{k}, \mathfrak{b}_{1}, \mathfrak{b}_{2}$ ):

$$
\mathfrak{k} \cap\left(\mathfrak{b}_{1}+\mathfrak{b}_{2}\right)=\mathfrak{k} \cap \mathfrak{b}_{1}+\mathfrak{k} \cap \mathfrak{b}_{2} .
$$

On peut alors trouver une base de $\mathfrak{g}$ qui soit adaptée aux différentes intersections. Dans ce cas, on peut adapter la forme à 8 couleurs en fonction des supplémentaires des intersections possibles.

Proposition 21. - Soient $f \in \mathfrak{k}^{\perp}$ et $\mathfrak{b}_{1}, \mathfrak{b}_{2}$ deux polarisations en $f$ telle que $\mathfrak{b}_{1}, \mathfrak{b}_{2}, \mathfrak{k}$ soient en position d'intersections normales. Alors le caractère construit est indépendant du choix de la polarisation (une fois choisi un supplémentaire de $\mathfrak{k}$ adapté à $\mathfrak{b}_{1}$ et $\mathfrak{b}_{2}$ ).

Démonstration. - Si les deux polarisations sont en position d'intersections normales on choisit un supplémentaire de $\mathfrak{k}$ adapté aux deux polarisations $\mathfrak{b}_{1}$ et $\mathfrak{b}_{2}$ simultanément. On dispose donc d'une algèbre de déformation $H_{\varepsilon, b_{1}, \mathfrak{b}_{2}}^{0}\left(\mathfrak{k}^{\perp}\right)$ (isomorphe à l'algèbre $S(\mathfrak{p})^{\mathfrak{k}}[[\varepsilon]]$ ).

Considérons la forme à 8 couleurs et les graphes de Kontsevich colorés. Sur la variété de configurations des points dans une demi-bande, plaçons aux coins les fonctions 1 et sur le côté borné la fonction $P \in H_{\varepsilon, b_{1}, \mathfrak{b}_{2}}^{0}\left(\mathfrak{k}^{\perp}\right)$ ( $c f$. (6.1) dessin de gauche). Les contributions de tous les graphes (pondérés par ces coefficients à 8 couleurs) représentent une fonction polynomiale sur $f+\left(\mathfrak{k}+\mathfrak{b}_{1}+\mathfrak{b}_{2}\right)^{\perp}$. Le résultat ne dépend pas de la position de la fonction $P$. Regardons les positions limites pour obtenir le résultat cherché. 


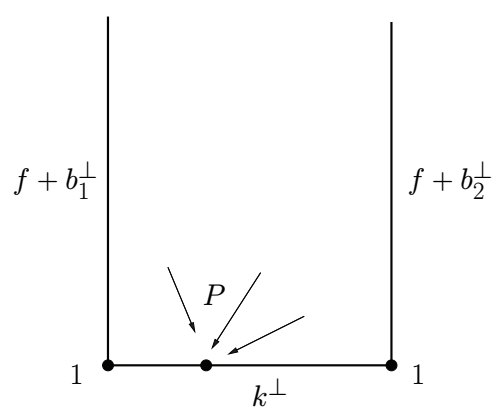

Indépendance via la forme 8 couleurs

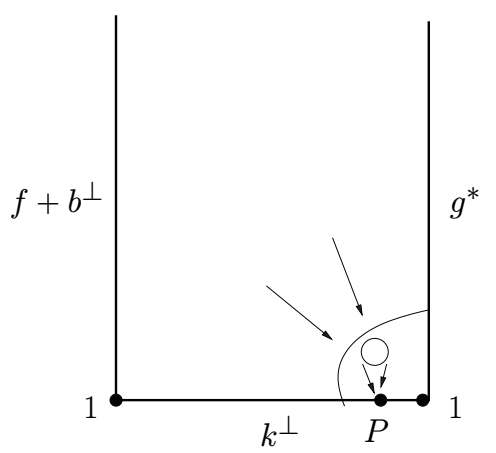

Calcul du caractère

En s'approchant du coin de droite, on obtient, grâce aux concentrations internes, un scalaire $1 \star x$ (et donc il n'y a pas de contributions pour les concentrations externes). On retrouve alors le caractère de la section précédente :

$$
\begin{aligned}
H_{\varepsilon, \mathfrak{b}_{1}}^{0}\left(\mathfrak{k}^{\perp}\right) & \rightarrow \mathbb{R}[[\varepsilon]] \\
P & \mapsto \underset{1}{\star} P .
\end{aligned}
$$

Lorsque la fonction $P$ s'approche de l'autre coin on trouve le caractère

$$
\begin{aligned}
H_{\varepsilon, \mathfrak{b}_{2}}^{0}\left(\mathfrak{k}^{\perp}\right) & \rightarrow \mathbb{R}[[\varepsilon]] \\
P & \mapsto P_{2}^{\star} 1 .
\end{aligned}
$$

On en déduit que les deux caractères construits sont identiques.

Remarque 11. - Cette construction fonctionne si on choisit comme sous-variétés coisotropes $f_{1}+\mathfrak{b}_{1}^{\perp}$ et $f_{2}+\mathfrak{b}_{2}^{\perp}$, où $f_{1}, f_{2}$ sont dans $\mathfrak{k}^{\perp}$ et vérifient la condition d'intersection :

$$
\left(f_{1}+\left(\mathfrak{k}+\mathfrak{b}_{1}\right)^{\perp}\right) \cap\left(f_{2}+\left(\mathfrak{k}+\mathfrak{b}_{2}\right)^{\perp}\right) \neq \varnothing .
$$

Si l'intersection ci-dessus est non vide, alors $f_{1}$ et $f_{2}$ sont dans la même $K$-orbite pour peu que la condition de Pukanszky soit vérifiée. En effet si la condition de Pukanszky ( $c f . \S 2.5$ ) est vérifiée on a

$$
\left(K \cap B_{1}\right) \cdot f_{1}=f_{1}+\left(\mathfrak{k}+\mathfrak{b}_{1}\right)^{\perp} \quad \text { et } \quad\left(K \cap B_{1}\right) \cdot f_{2}=f_{2}+\left(\mathfrak{k}+\mathfrak{b}_{2}\right)^{\perp} .
$$

Alors $f_{1}$ et $f_{2}$ sont conjuguées par $K$ dans $\mathfrak{k}^{\perp}$.

\subsection{Applications au cas des polarisations $\sigma$-stables}

On applique la construction ci-dessus, dans le cas des paires symétriques qui admettent de manière générique des polarisations $\sigma$-stables pour les formes linéaires dans $\mathfrak{k}^{\perp}$.

On spécialise ensuite nos constructions en $\varepsilon=1$ pour les polynômes en $\varepsilon$ (au lieu des séries formelles en $\varepsilon$ ). 
Exemples classiques. - Les paires symétriques pour lesquelles on peut construire des polarisations $\sigma$-stables sont proches heuristiquement des algèbres de Lie. Voici quelques exemples (cf. $[33,35])$.

1. Les petites paires symétriques comme dans $\S 2.4$,

2. le cas de paires symétriques nilpotentes,

3. le cas des paires symétriques de Alekseev-Meinrenken,

4. le cas des algèbres de Lie considérées comme des espaces symétriques,

5. les paires symétriques de Takiff $\mathfrak{g} \oplus \mathfrak{g} \otimes T$.

Indépendance du caractère. - Lorsque l'on sait construire des polarisations $\sigma$-stables, les supplémentaires s'imposent pour $\mathfrak{b} /(\mathfrak{b} \cap \mathfrak{k}$ ) ainsi que $\mathfrak{g} /(\mathfrak{k}+\mathfrak{b})$; on choisit $\mathfrak{b} \cap \mathfrak{p}$ et $\mathfrak{p} / \mathfrak{p} \cap \mathfrak{b}$ (un supplémentaire de $\mathfrak{p} \cap \mathfrak{b}$ dans $\mathfrak{p}$ ). L'algèbre de réduction pour $\mathfrak{k}^{\perp}$ est alors tout simplement $S(\mathfrak{p})^{\mathfrak{k}}$ munie du produit de $\underset{\mathrm{CF}}{\star}$, car tous les espaces considérés sont $\sigma$-stables.

Différents choix de polarisations $\sigma$-stables vont être en position d'intersection normale. Ainsi d'après la proposition 21, le caractère construit est indépendant de la polarisation $\sigma$-stable; notons-le

$$
P \mapsto \gamma(P)(f)
$$

Par ailleurs cette construction est équivariante par rapport à l'action linéaire de $K$, donc le caractère construit ne dépend que de la $K$-orbite de $f$. On en déduit la proposition suivante (cf. $[33,35])$.

Proposition 22. - S'il existe génériquement des polarisations $\sigma$-stables, alors la construction ci-dessus définit un caractère de $\left(S(\mathfrak{p})^{\mathfrak{k}}, \stackrel{\star}{\mathrm{CF}}\right)$. Ce caractère ne dépend pas du choix de la polarisation $\sigma$-stable. Il est constant sur la $K$-orbite et polynomial en $f$.

Démonstration. - Le caractère ne dépend pas du choix de la polarisation $\sigma$-stable, car on est automatiquement en situation d'intersection normale et l'algèbre de réduction ne dépend pas des choix des supplémentaires, car ils sont pris dans $\mathfrak{p}$.

La dépendance polynomiale en $\mathfrak{b}$ est claire, tant que l'on reste sur des ouverts où $f$ admet des polarisations génériques. On applique la méthode de Duflo-Conze [11] pour conclure. L'ensemble des $(f, \mathfrak{b})$ avec $f \in \mathfrak{k}^{\perp}$ et $\mathfrak{b}$ sous-algèbre $\sigma$-stable subordonnée à $f$ et de dimension générique, tels que $1 \star P$ soit scalaire dans $f+(\mathfrak{k}+\mathfrak{b})^{\perp}$ forme un ensemble rationnel. La fibre étant projective, on conclut alors que le caractère rationnel est en fait polynomial et $K$-invariant.

Isomorphisme de Rouvière. - L'homomorphisme $\gamma$ est un isomorphisme de $S(\mathfrak{p})^{\mathfrak{k}}$ muni du produit $\underset{C F}{\star} \operatorname{sur} S(\mathfrak{p})^{\mathfrak{k}}$ muni du produit standard, car le terme de plus haut degré est clairement l'identité sur les éléments homogènes.

En fait on a le résultat plus fort suivant (démontré dans [35] par la méthode des orbites, voir aussi [1, 32]).

$4^{\mathrm{e}}$ SÉRIE - TOME $41-2008-\mathrm{N}^{\mathrm{o}} 5$ 
ThÉorème 8. - Dans le cas des paires symétriques, avec polarisations génériques $\sigma$-stables, l'homomorphisme $\gamma$ vaut l'identité. En conséquence l'application ${ }^{(43)}$

$$
P \mapsto \beta\left(\partial\left(J^{\frac{1}{2}}\right) P\right)
$$

est un isomorphisme d'algèbres de $S(\mathfrak{p})^{\mathfrak{k}} \operatorname{sur}(U(\mathfrak{g}) / U(\mathfrak{g}) \cdot \mathfrak{k})^{\mathfrak{k}}$.

Démonstration. - Soit $f \in \mathfrak{k}^{\perp}, \mathfrak{b}$ polarisation $\sigma$-stable en $f$. On considère dans le diagramme de la bi-quantification la situation du triplet : $f+\mathfrak{b}^{\perp}, \mathfrak{k}^{\perp}$ et $\mathfrak{g}^{*}$. On place les fonctions 1 aux deux coins et la fonction $P \in S(\mathfrak{p})^{\mathfrak{k}}$ sur l'axe horizontal ( $c f$. (6.1) dessin de droite).

Lorsque $P$ se rapproche du coin $f+\mathfrak{b}^{\perp}$, on retrouve le caractère recherché.

Lorsque $P$ se rapproche du coin correspondant à $\mathfrak{g}^{*}$, on retrouve dans les strates qui se concentrent (sur l'axe horizontal) la situation des paires symétriques à savoir $P \star 1=B(P)$, où $B$ désigne l'opérateur associé aux roues pures attachées sur l'axe $\mathfrak{k}^{\perp}(c f . \S 4.1)$.

Considérons les contributions des graphes extérieurs et montrons qu'elles sont triviales. Les arêtes qui arrivent sur le coin sont colorées par les couleurs +++ ou -++ . En fait pour la fonction d'angle à 8 couleurs, on a $\phi_{-++}(p, q)=0$ si $q$ est dans le coin. Par conséquent les arêtes qui arrivent sur le coin sont d'une seule couleur $:+++$. Cette couleur correspond à une dérivée dans la direction $(\mathfrak{p} / \mathfrak{p} \cap \mathfrak{b})^{*}=(\mathfrak{k}+\mathfrak{b})^{\perp}$. Or la fonction que l'on dérive est $B(P)$, qui est $\mathfrak{k}$-invariante et donc constante sur $f+(\mathfrak{k}+\mathfrak{b})^{\perp}$. De plus l'opérateur est évalué en $\xi \in f+(\mathfrak{k}+\mathfrak{b})^{\perp}$, par conséquent ses dérivées sont nulles. Le caractère construit dans la proposition 22 vaut donc $B(P)(f)$.

La série $B$ est universelle et s'écrit sous la forme :

$$
\exp \left(\sum w_{n} \operatorname{tr}_{\mathfrak{p}}(\operatorname{ad} X)^{2 n}\right)
$$

où $w_{n}$ sont des constantes universelles. On en déduit que l'application de

$$
P \mapsto B(P)
$$

de $\left(S(\mathfrak{p})^{\mathfrak{k}}, \underset{\mathrm{CF}}{\star}=\underset{\text { Rou }}{\sharp}\right) \operatorname{sur}\left(S(\mathfrak{p})^{\mathfrak{k}}, \cdot\right)$ est un isomorphisme d'algèbres pour les paires symétriques qui admettent génériquement des polarisations $\sigma$-stables. Pour terminer la preuve du théorème il suffit de montrer que l'on a $B=1$.

Proposition 23. - La série universelle $B(X)$ qui intervient pour les paires symétriques vaut 1 .

Démonstration. - D'après [35] lorsque génériquement on sait construire des polarisations $\sigma$-stables, le produit de Rouvière (qui vaut aussi le star-produit $\underset{\mathrm{CF}}{\star}$ ) et le produit standard coïncident sur $S(\mathfrak{p})^{\mathfrak{k}}$.

Si on avait $B \neq 1$ alors on disposerait d'un isomorphisme d'algèbres non trivial, pour $S(\mathfrak{p})^{\mathfrak{k}}$ muni du produit standard (pour ce genre de paires symétriques). Il existerait $n>0$ tel que $\operatorname{tr}_{\mathfrak{p}}(\operatorname{ad} X)^{2 n}$ serait une dérivation de $S(\mathfrak{p})^{\mathfrak{k}}$, ce qui n'est pas vrai sur des exemples ${ }^{(44)}$.

La série universelle $B$ vaut 1 , ce qui justifie la formule pour l'écriture des opérateurs différentiels invariants en coordonnées exponentielles du $\S 4.3$.

(43) Ici $\beta$ désigne la symétrisation.

(44) Par exemple pour $s l(2)$ considérée comme paire symétrique. 
6.3.0.1. Remarque 12. - Dans le cas des paires symétriques de Takiff $\mathfrak{g} \oplus \mathfrak{g} \otimes T$, avec $\mathfrak{g}$ une paire symétrique et $T^{2}=0$, on a

$$
E_{\mathfrak{g} \oplus \mathfrak{g} \otimes T}\left(X+X^{\prime} \otimes T, Y+Y^{\prime} \otimes T\right)=E_{\mathfrak{g}}^{2}(X, Y) .
$$

On sait toutefois que, dans ce contexte, l'homomorphisme d'Harish-Chandra coïncide avec l'isomorphisme de Rouvière car on peut construire des polarisations $\sigma$-stables ( $c f$. $\S 6.3$ ). Ceci montre que la formule de Rouvière peut réaliser un isomorphisme d'algèbres sans que la fonction $E_{\mathfrak{g} \oplus \mathfrak{g} \otimes T}$ soit égale à 1. La question pertinente est donc de savoir si cette fonction est homotope à 1 modulo les champs $\mathfrak{k} \oplus \mathfrak{k}_{T}$-adjoints.

\section{Remerciements}

A. S. Cattaneo remercie, d'une part, la FNS pour son soutien financier, à travers la bourse $n^{\circ} 200020-107444 / 1$ et, d'autre part, l'IHÉS où il a séjourné pendant la réalisation de ce travail. Par ailleurs ce projet a été partiellement financé par l'Union Européenne à travers le projet FP6 Marie Curie RTN ENIGMA (numéro de contrat MRTN-CT-2004-5652). La recherche de $\mathrm{Ch}$. Torossian a été financée par le CNRS.

Les deux auteurs remercient particulièrement G. Felder, D. Indelicato, B. Keller et D. Manchon pour les discussions fructueuses qu'ils ont eues durant l'élaboration de ce travail, ainsi que Jim Stasheff et F. Rouvière pour leurs commentaires concernant une version préliminaire de cet article et le rapporteur anonyme pour ses nombreuses remarques et suggestions qui ont permis l'amélioration de ce mémoire.

\section{BIBLIOGRAPHIE}

[1] A. Alekseev, E. Meinrenken, Lie theory and the Chern-Weil homomorphism, Ann. Sci. École Norm. Sup. 38 (2005), 303-338.

[2] A. Alekseev, E. Meinrenken, On the Kashiwara-Vergne conjecture, Invent. Math. 164 (2006), 615-634.

[3] M. Andler, S. Sahi, C. Torossian, Convolution of invariant distributions : proof of the Kashiwara-Vergne conjecture, Lett. Math. Phys. 69 (2004), 177-203.

[4] D. Arnal, D. Manchon, M. Masmoudi, Choix des signes pour la formalité de M. Kontsevich, Pacific J. Math. 203 (2002), 23-66.

[5] A. Baklouti, H. Fujiwara, Commutativité des opérateurs différentiels sur l'espace des représentations restreintes d'un groupe de Lie nilpotent, J. Math. Pures Appl. 83 (2004), 137-161.

[6] A. Baklouti, J. Ludwig, Invariant differential operators on certain nilpotent homogeneous spaces, Monatsh. Math. 134 (2001), 19-37.

[7] A. S. Cattaneo, G. Felder, Coisotropic submanifolds in Poisson geometry and branes in the Poisson sigma model, Lett. Math. Phys. 69 (2004), 157-175.

[8] A. S. Cattaneo, G. Felder, Relative formality theorem and quantisation of coisotropic submanifolds, Adv. Math. 208 (2007), 521-548.

$4{ }^{\mathrm{e}}$ SÉRIE - TOME $41-2008-\mathrm{N}^{\circ} 5$ 
[9] A. S. Cattaneo, B. Keller, C. Torossian, A. Bruguières, Déformation, quantification, théorie de Lie, Panoramas et Synthèses 20, Soc. Math. France, 2005.

[10] L. Corwin, F. P. Greenleaf, Commutativity of invariant differential operators on nilpotent homogeneous spaces with finite multiplicity, Comm. Pure Appl. Math. 45 (1992), 681-748.

[11] M. Duflo, Opérateurs différentiels bi-invariants sur un groupe de Lie, Ann. Sci. École Norm. Sup. 10 (1977), 265-288.

[12] M. Duflo, Opérateurs différentiels invariants sur un espace symétrique, C. R. Acad. Sci. Paris 289 (1979), 135-137.

[13] M. Duflo, Open problems in representation theory of Lie groups, in Conference on Analysis on homogeneous spaces, August 25-30, Kataka, Japon (T. Oshima, éd.), 1986.

[14] H. Fujiwara, G. Lion, B. Magneron, S. Mehdi, A commutativity criterion for certain algebras of invariant differential operators on nilpotent homogeneous spaces, Math. Ann. 327 (2003), 513-544.

[15] A. Iserles, Solving linear ordinary differential equations by exponentials of iterated commutators, Numer. Math. 45 (1984), 183-199.

[16] A. Iserles, Expansions that grow on trees, Notices Amer. Math. Soc. 49 (2002), 430 440.

[17] M. Kashiwara, M. Vergne, The Campbell-Hausdorff formula and invariant hyperfunctions, Invent. Math. 47 (1978), 249-272.

[18] F. Knop, A Harish-Chandra homomorphism for reductive group actions, Ann. of Math. 140 (1994), 253-288.

[19] M. Kontsevich, Deformation quantization of Poisson manifolds, Lett. Math. Phys. 66 (2003), 157-216.

[20] T. H. Koornwinder, Invariant differential operators on nonreductive homogeneous spaces, Afdeling Zuivere Wiskunde 153, Mathematisch Centrum, 1981.

[21] A. Lichnerowicz, Opérateurs différentiels invariants sur un espace symétrique, $C$. $R$. Acad. Sci. Paris 256 (1963), 3548-3550.

[22] W. Magnus, On the exponential solution of differential equations for a linear operator, Comm. Pure Appl. Math. 7 (1954), 649-673.

[23] D. Manchon, Poisson bracket, deformed bracket and gauge group actions in Kontsevich deformation quantization, Lett. Math. Phys. 52 (2000), 301-310.

[24] M. Pevzner, C. Torossian, Isomorphisme de Duflo et la cohomologie tangentielle, J. Geom. Phys. 51 (2004), 487-506.

[25] F. Rouvière, Espaces symétriques et méthode de Kashiwara-Vergne, Ann. Sci. École Norm. Sup. 19 (1986), 553-581.

[26] F. Rouvière, Invariant analysis and contractions of symmetric spaces. I, Compositio Math. 73 (1990), 241-270.

[27] F. Rouvière, Invariant analysis and contractions of symmetric spaces. II, Compositio Math. 80 (1991), 111-136. 
[28] F. Rouvière, Une propriété de symétrie des espaces symétriques, C. R. Acad. Sci. Paris Sér. I Math. 313 (1991), 5-8.

[29] F. Rouvière, Fibrés en droites sur un espace symétrique et analyse invariante, J. Funct. Anal. 124 (1994), 263-291.

[30] L. G. Ryвnikov, Structure of the center of the algebra of invariant differential operators on certain Riemannian homogeneous spaces, Transform. Groups 9 (2004), 381397.

[31] B. Shoik het, On the Duflo formula for $L_{\infty}$-algebras and $Q$-manifolds, prépublication arXiv:math.QA/9812009.

[32] C. Torossian, Opérateurs différentiels invariants sur les espaces symétriques. I et II, J. Funct. Anal. 117 (1993), 118-173 et 174-214.

[33] C. Torossian, Sur la conjecture combinatoire de Kashiwara-Vergne, J. Lie Theory 12 (2002), 597-616.

[34] C. Torossian, Méthodes de Kashiwara-Vergne-Rouvière pour les espaces symétriques, in Noncommutative harmonic analysis, Progr. Math. 220, Birkhäuser, 2004, 459-486.

[35] C. Torossian, Paires symétriques orthogonales et isomorphisme de Rouvière, J. Lie Theory 15 (2005), 79-87.

[36] C. Torossian, La conjecture de Kashiwara-Vergne (d'après Alekseev-Meinrenken), Sém. Bourbaki (2006/07), exp. no 980, Astérisque 317 (2008), 441-465.

[37] M. Vergne, Le centre de l'algèbre enveloppante et la formule de Campbell-Hausdorff, C. R. Acad. Sci. Paris Sér. I Math. 329 (1999), 767-772.

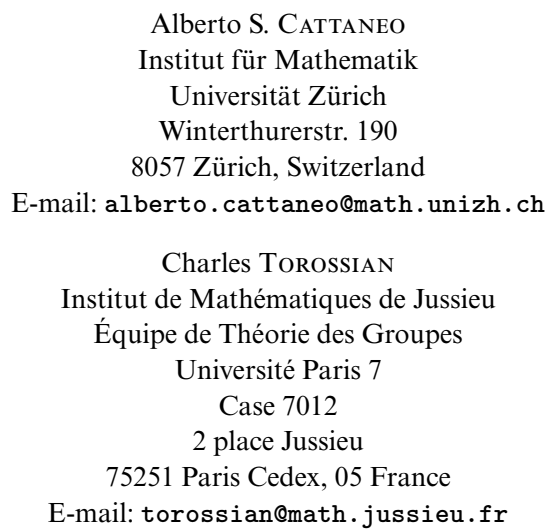

